Online supplement to:

Kinematics of the Amanos Fault, southern Turkey, from Ar-Ar dating of offset Pleistocene basalt flows: transpression between the African and Arabian plates

by

Ali Seyrek, Tuncer Demir, Malcolm Pringle, Sema Yurtmen, Rob Westaway, Anthony Beck, George Rowbotham

Prepared by Rob Westaway, The Open University, June 2006 based on data supplied by Bill Olszewski, Massachusetts Institute of Technology. 
Sample 01TR53 
Data Tables 
Clair
MIT, Cambridge, USA

\begin{tabular}{|c|c|c|c|c|c|c|c|c|c|c|c|}
\hline $\begin{array}{l}\text { Procedu } \\
\text { Blanks }\end{array}$ & & 36Ar & $1 \sigma$ & $37 \mathrm{Ar}$ & $1 \sigma$ & 38Ar & $1 \sigma$ & $39 \mathrm{Ar}$ & $1 \sigma$ & $40 \mathrm{Ar}$ & $1 \sigma$ \\
\hline $\begin{array}{l}\text { 5F041P.01 } \\
\text { 5F041P.02 }\end{array}$ & $\begin{array}{l}770^{\circ} \mathrm{C} \\
870^{\circ} \mathrm{C}\end{array}$ & $\begin{array}{l}0.000242 \\
0.000243\end{array}$ & $\begin{array}{l}0.000017 \\
0.000017\end{array}$ & $\begin{array}{l}0.000242 \\
0.000243\end{array}$ & $\begin{array}{l}0.000029 \\
0.000029\end{array}$ & $\begin{array}{l}0.000138 \\
0.000139\end{array}$ & $\begin{array}{l}0.000023 \\
0.000023\end{array}$ & $\begin{array}{l}0.000424 \\
0.000426\end{array}$ & $\begin{array}{l}0.000045 \\
0.00004\end{array}$ & $\begin{array}{l}0.040000 \\
0.040000\end{array}$ & $\begin{array}{l}0.000125 \\
0.000125\end{array}$ \\
\hline $\begin{array}{l}5=447.02 \\
\text { 5F04P.02 }\end{array}$ & $\begin{array}{l}870^{\circ} \mathrm{C} \\
9\end{array}$ & $\begin{array}{l}0.000243 \\
0.000243\end{array}$ & $\begin{array}{l}0.000017 \\
0.000017\end{array}$ & $\begin{array}{l}0.000243 \\
0.000244\end{array}$ & $\begin{array}{l}0.000029 \\
0.000029\end{array}$ & $\begin{array}{l}0.000139 \\
0.000139\end{array}$ & $\begin{array}{l}0.000023 \\
0.000023\end{array}$ & $\begin{array}{l}0.000426 \\
0.000426\end{array}$ & $\begin{array}{l}0.000045 \\
0.000045\end{array}$ & $\begin{array}{l}0.040000 \\
0.040000\end{array}$ & $\begin{array}{l}0.0000125 \\
0.000125\end{array}$ \\
\hline 5F041P.04 & $1020^{\circ} \mathrm{C}$ & 0.000243 & 0.000017 & 0.000245 & 0.000029 & 0.000139 & 0.000023 & 0.000427 & 0.000045 & 0.040000 & 0.000125 \\
\hline 5F041P.05 & $1070^{\circ} \mathrm{C}$ & 0.000244 & 0.000017 & 0.000245 & 0.000029 & 0.000140 & 0.000023 & 0.000428 & 0.000045 & 0.040000 & 0.000125 \\
\hline 5F041P.06 & $1120^{\circ} \mathrm{C}$ & 0.000244 & 0.000017 & 0.000246 & 0.000029 & 0.000140 & 0.000023 & 0.000428 & 0.000045 & 0.040000 & 0.000125 \\
\hline $\begin{array}{l}5 F 041 P .07 \\
5041\end{array}$ & $1190^{\circ} \mathrm{C}$ & 0.000244 & 0.000017 & 0.000247 & $\begin{array}{l}0.000029 \\
\end{array}$ & 0.000140 & 0.000023 & 0.000429 & 0.000045 & 0.040000 & 0.000125 \\
\hline 5F041P.09 & $1370^{\circ} \mathrm{C}$ & $\begin{array}{l}0.000244 \\
0.000245\end{array}$ & $\begin{array}{l}0.000017 \\
0.000017\end{array}$ & $\begin{array}{l}0.000247 \\
0.000248\end{array}$ & $\begin{array}{l}0.000029 \\
0.000029\end{array}$ & $\begin{array}{l}0.0000140 \\
0.000141\end{array}$ & $\begin{array}{l}0.0000023 \\
0.000023\end{array}$ & $\begin{array}{l}0.0000430 \\
0.000430\end{array}$ & $\begin{array}{l}0.000045 \\
0.000045\end{array}$ & $\begin{array}{l}0.0440000 \\
0.0400000\end{array}$ & $\begin{array}{l}0.000125 \\
0.000125\end{array}$ \\
\hline 5F041P.10 & $1670^{\circ} \mathrm{C}$ & 0.000245 & 0.000017 & 0.000249 & 0.000029 & 0.000141 & 0.000023 & 0.000431 & 0.000045 & 0.040000 & 0.000125 \\
\hline
\end{tabular}




\begin{tabular}{|c|c|c|c|c|c|c|c|c|c|c|c|c|c|c|c|c|c|c|c|c|c|}
\hline $\begin{array}{l}\text { Intercep } \\
\text { Values }\end{array}$ & & $36 \mathrm{Ar}$ & $1 \sigma$ & 12 & & $37 \mathrm{Ar}$ & $1 \sigma$ & $\mathrm{r} 2$ & & 38Ar & $1 \sigma$ & $\mathrm{r}^{2}$ & & $39 \mathrm{Ar}$ & $1 \sigma$ & $\mathrm{r} 2$ & & $40 \mathrm{Ar}$ & $1 \sigma$ & r2 & \\
\hline $\begin{array}{l}5 F 041 P .01 \\
5 F 41 P(2)\end{array}$ & $\begin{array}{l}770^{\circ} \mathrm{C} \\
870^{\circ} \mathrm{C}\end{array}$ & 0.002142 & 0.000016 & 0.8499 & LIN \# 17 & 0.007109 & 0.000042 & 0.0600 & $\begin{array}{l}\text { LIN \# } 12 \\
\text { EXP \# } 1612\end{array}$ & 0.000731 & 0.000018 & 0.1205 & LIN \# 12 & 0.013320 & 0.000056 & 0.9870 & LIN \#12 & 0.567545 & 0.000415 & 0.9864 & LIN \# 1910 \\
\hline $\begin{array}{l}5 F 041 P .02 \\
5 F 041.03\end{array}$ & $\begin{array}{l}870^{\circ} \mathrm{C} \\
970^{\circ} \mathrm{C}\end{array}$ & $\begin{array}{l}0.0003429 \\
0.003196\end{array}$ & $\begin{array}{l}0.000024 \\
0.000031\end{array}$ & $\begin{array}{l}0.8696 \\
0.7665\end{array}$ & $\begin{array}{l}\operatorname{LN} \# 1 \\
\operatorname{LIN} \# 124\end{array}$ & $\begin{array}{l}0.029194 \\
0.065487\end{array}$ & $\begin{array}{l}0.0000060 \\
0.000049\end{array}$ & $\begin{array}{l}0.9912 \\
0.9990\end{array}$ & $\begin{array}{l}\operatorname{EXP} \# 1612 \\
\operatorname{EXP} \# 46\end{array}$ & $\begin{array}{l}0.0001714 \\
0.003253\end{array}$ & $\begin{array}{l}0.000050 \\
0.000032\end{array}$ & $\begin{array}{l}0.4042 \\
0.6265\end{array}$ & $\begin{array}{l}\text { LIN \#1114 } \\
\text { LIN \#12 }\end{array}$ & $\begin{array}{l}0.0669242 \\
0.207547\end{array}$ & $\begin{array}{l}0.000062 \\
0.000089\end{array}$ & $\begin{array}{l}0.9925 \\
0.9995\end{array}$ & $\begin{array}{l}\text { EXP \#1 } 148 \\
\text { EXP \# } 1013\end{array}$ & $\begin{array}{l}0.9377732 \\
0.924142\end{array}$ & $\begin{array}{l}0.000422 \\
0.000369\end{array}$ & $\begin{array}{l}0.9835 \\
0.9772\end{array}$ & $\begin{array}{l}\operatorname{LIN} \# 1 \\
\operatorname{EXP} \# 110\end{array}$ \\
\hline 5F041P.04 & $1020^{\circ} \mathrm{C}$ & 0.002257 & 0.000019 & 0.7066 & LIN \# 110 & 0.068463 & 0.000068 & 0.9979 & LIN \# & 0.004027 & 0.000040 & 0.7729 & LIN \# 1 & 0.283535 & 0.000254 & 0.9983 & LIN \# 1 & 0.668835 & 0.000309 & 0.9890 & LIN \# 15 \\
\hline 5F041P.05 & $1070^{\circ} \mathrm{C}$ & 0.002108 & 0.000022 & 0.3859 & LIN \# 1 & 0.080277 & 0.000060 & 0.9991 & EXP \# 312 & 0.004942 & 0.000025 & 0.9471 & LIN \# 1 & 0.358062 & 0.000188 & 0.9994 & EXP \# 1 & 0.660300 & 0.000347 & 0.9942 & LIN \# 1349 \\
\hline 5F041P.06 & $1120^{\circ} \mathrm{C}$ & 0.002083 & 0.000020 & 0.3239 & LIN \# 1 & 0.076794 & 0.000059 & 0.9990 & EXP \# 4513 & & 0.000025 & 0.9367 & & & & 0.9993 & & & 0.000145 & 0.9987 & EXP \# 127 \\
\hline 5F041P.07 & $1190^{\circ} \mathrm{C}$ & 0.002867 & 0.000018 & 0.8619 & LIN \# 1 & 0.077918 & 0.000060 & 0.9992 & EXP \# 57912 & 0.004333 & 0.000015 & 0.9806 & LIN \# 15 & 0.284845 & 0.000128 & 0.9997 & EXP \# 1611 & 0.840621 & 0.000331 & 0.7893 & LIN \# 1712 \\
\hline $5 F 041 P .08$ & $1270^{\circ} \mathrm{C}$ & 0.003070 & 0.000027 & 0.7732 & LIN \# 12 & 0.071759 & 0.000051 & 0.9991 & EXP \# 512 & 0.002955 & 0.000025 & 0.8301 & LIN \# 1815 & 0.171948 & 0.000104 & 0.9991 & EXP \# 781115 & 0.869922 & 0.000340 & 0.9609 & $\begin{array}{l}\text { LIN \# } 1915 \\
\text { LYP }\end{array}$ \\
\hline 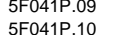 & $\begin{array}{l}1370^{\circ} \mathrm{C} \\
1670^{\circ} \mathrm{C}\end{array}$ & $\begin{array}{l}0.004020 \\
0.041226\end{array}$ & $\begin{array}{l}0.000024 \\
0.000025\end{array}$ & 0.8504 & $\begin{array}{l}\operatorname{LN} \# 1 \\
\operatorname{EXP} \# 610\end{array}$ & $\begin{array}{l}0.073577 \\
1.478912\end{array}$ & $\begin{array}{l}0.000092 \\
0.000533\end{array}$ & 0.9968 & $\begin{array}{l}\text { EXP \#2 } \\
\text { EXP\#\# }\end{array}$ & $\begin{array}{l}0.002970 \\
0.013389\end{array}$ & 0.000033 & 0.6696 & $\begin{array}{l}\text { LIN \# } \\
\text { LN \# }\end{array}$ & 0.150901 & 0.000141 & 0.9980 & $\begin{array}{l}\text { EXP \# } 123111 \\
\text { EXP \#1711 }\end{array}$ & $\begin{array}{r}1.140532 \\
11.368984\end{array}$ & $\begin{array}{l}0.000330 \\
0.02405\end{array}$ & $\begin{array}{l}0.9983 \\
0.997\end{array}$ & $\begin{array}{l}\operatorname{EXP} \# 123411 \\
\operatorname{EXP} \# 11011\end{array}$ \\
\hline
\end{tabular}




\begin{tabular}{|c|c|c|c|c|c|c|c|c|c|c|c|c|c|c|c|c|c|c|c|c|c|c|c|}
\hline \multicolumn{2}{|c|}{$\begin{array}{l}\text { Sample } \\
\text { Parameters }\end{array}$} & Sample & Material & Location & Analyst & 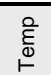 & $\begin{array}{l}\text { Standard } \\
\text { (in Ma) }\end{array}$ & $\% 1 \sigma$ & $J$ & $\% 1 \sigma$ & Fract & $\% 1 \sigma$ & $\begin{array}{l}\text { Volume } \\
\text { Corr. }\end{array}$ & $\begin{array}{c}\text { Sensitivity } \\
\text { (mol/vol) }\end{array}$ & बึ & $\begin{array}{l}\text { 吉 } \\
\sum_{\Sigma}^{\circ}\end{array}$ & 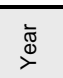 & 호 & $\sum$ & 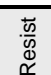 & Irradiation & Project & $\begin{array}{l}\text { Standard } \\
\text { Name }\end{array}$ \\
\hline $\begin{array}{l}5 F 041 P .01 \\
5041 P^{2}\end{array}$ & $770^{\circ} \mathrm{C}$ & $\begin{array}{l}01 \text { TR53 B20 } \\
01 \text { T553 B20 }\end{array}$ & bas grndmss & Iestaway Volcanii & $\mathrm{msp}$ & 770 & 28.34 & 0.01 & 0.0003028 & 0.3 & 1.007 & 0.2 & 1 & $6.000 E-14$ & 30 & 07 & 2005 & 04 & 44 & 001 & cl155- & Westaway & tcr-2a \\
\hline 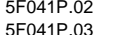 & $\begin{array}{l}870^{\circ} \mathrm{C} \\
970^{\circ} \mathrm{C}\end{array}$ & 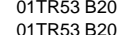 & $\begin{array}{l}\text { bas grndmss } \\
\text { bas arndmss }\end{array}$ & $\begin{array}{l}\text { 'estaway Volcanit } \\
\text { lestaway Volcanit }\end{array}$ & $\begin{array}{c}\text { msp } \\
\text { msp }\end{array}$ & 870 & $\begin{array}{l}28.34 \\
2834\end{array}$ & 0.01 & $\begin{array}{l}0.0003028 \\
0.0003028\end{array}$ & $\begin{array}{l}0.3 \\
0.3\end{array}$ & 1.007 & 0.2 & 1 & $6.000 E-14$ & 30 & 07 & 2005 & 05 & 42 & 001 & cl155- & Westaway & tcr-2a \\
\hline $\begin{array}{l}\text { 5FF41P.03 } \\
\text { 5044P. }\end{array}$ & $\begin{aligned} 970^{\circ} \mathrm{C} \\
1020^{\circ} \mathrm{C}\end{aligned}$ & 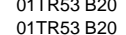 & $\begin{array}{l}\text { bas grndmss } \\
\text { bas grndmss }\end{array}$ & $\begin{array}{l}\text { lestaway Volcanit } \\
\text { lestaway Volcanit }\end{array}$ & $\begin{array}{l}\text { msp } \\
\text { msp }\end{array}$ & $\begin{array}{r}970 \\
1020\end{array}$ & $\begin{array}{l}28.34 \\
28.34\end{array}$ & 0.01 & $\begin{array}{l}0.00030028 \\
0.000328\end{array}$ & 0.3 & $\begin{array}{l}.007 \\
1.007\end{array}$ & 0.2 & 1 & $\begin{array}{l}6.000 E-14 \\
6600 E-14\end{array}$ & $\begin{array}{l}30 \\
30\end{array}$ & 07 & 2005 & 06 & 16 & 001 & $\begin{array}{l}\text { clis5- } \\
c 155-\end{array}$ & Westaway & tcr-2a \\
\hline 5F041P.05 & $1070^{\circ} \mathrm{C}$ & $\begin{array}{l}01 \text { TR53 B20 } \\
0\end{array}$ & bas grndmss & lestaway Volcanit & $\begin{array}{l}\text { misp } \\
\text { msp }\end{array}$ & 1070 & $\begin{array}{l}28.34 \\
28.34\end{array}$ & 0.01 & 0.0003028 & $\begin{array}{l}0.3 \\
0.3\end{array}$ & 1.00 & $\begin{array}{l}0.2 \\
0.2\end{array}$ & 1 & 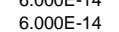 & 30 & 07 & 2005 & $\begin{array}{l}00 \\
07\end{array}$ & $\begin{array}{l}49 \\
23\end{array}$ & 001 & cl155- & Westaway & $\begin{array}{l}\text { tcr-2a } \\
\text { tcr-2a }\end{array}$ \\
\hline $5=041 P 06$ & $1120^{\circ} \mathrm{C}$ & $01 \mathrm{TR} 53 \mathrm{~B} 20$ & bas grndmss & Iestaway Volcanit & msp & 1120 & 2834 & 0.01 & 00003028 & 0.3 & 100 & 2 & 1 & $6000 E-14$ & 30 & 07 & 2005 & 07 & 56 & 001 & (c155. & Woctavar & \\
\hline 5 5E41P07 & $110^{\circ} \mathrm{c}$ & $01 \mathrm{TR} 53 \mathrm{~B} 20$ & $\begin{array}{l}\text { Was andmss } \\
\text { bas andme }\end{array}$ & lestaway Volcani & $\begin{array}{ll}\text { mss } & \\
\text { ms }\end{array}$ & 1190 & 2834 & 01 & - 00003028 & 03 & 1007 & 02 & 1 & 6000 -14 & 30 & 07 & 2005 & 08 & 30 & 001 & & $\begin{array}{l}\text { Westavady } \\
\text { Wectway }\end{array}$ & 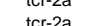 \\
\hline 5 5F41P 08 & ${ }_{1270}^{\circ} \mathrm{C}$ & $01 \mathrm{TT} 53 \mathrm{~B} 20$ & $\begin{array}{l}\text { bas ardmss } \\
\text { bas se }\end{array}$ & lestaway Volcani & ms & 1270 & 2834 & 0.01 & $\begin{array}{l}0.0003028 \\
0.003\end{array}$ & 0.3 & 1.007 & 02 & 1 & $6000-14$ & 30 & 07 & 2005 & 09 & 04 & - & d11 & Wectavay & 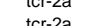 \\
\hline 5F041P.09 & $1370^{\circ} \mathrm{C}$ & $01 \mathrm{TR} 53 \mathrm{~B} 20$ & bas grndmss & lestaway Volcani & msp & 1370 & 28.34 & 0.01 & 0.0003028 & 0.3 & 1.007 & 0.2 & 1 & $6.000 E-14$ & 30 & 07 & 2005 & 09 & 37 & 001 & cl155- & Westaway & $\begin{array}{l}\text { tor-2a } \\
\text { tor }\end{array}$ \\
\hline 5F041P.10 & $1670^{\circ} \mathrm{C}$ & $01 \mathrm{TR} 53 \mathrm{~B} 20$ & bas grndmss & lestaway Volcanii & msp & 1670 & 28.34 & 0.01 & 0.0003028 & 0.3 & 1.007 & 0.2 & 1 & $6.000 E-14$ & 30 & 07 & 2005 & 10 & 11 & 001 & cl155- & Westaway & tcr-2a \\
\hline
\end{tabular}


Clair

MIT, Cambridge, USA

\begin{tabular}{|c|c|c|c|c|c|c|c|c|c|c|c|c|c|c|c|c|c|c|c|c|c|c|c|}
\hline \multicolumn{2}{|c|}{$\begin{array}{l}\text { Irradiation } \\
\text { Constants }\end{array}$} & 40/36(a) & $\% 1 \sigma$ & $38 / 36$ (a) & $\% 1 \sigma$ & 39/37(ca) & $\% 1 \sigma$ & 38/37(ca) & $\% 1 \sigma$ & $36 / 37$ (ca) & $\% 1 \sigma$ & $40 / 39(k)$ & $\% 1 \sigma$ & $38 / 39(\mathrm{k})$ & $\% 1 \sigma$ & $36 / 38$ (cl) & $\% 1 \sigma$ & $\mathrm{K} / \mathrm{Ca}$ & $\% 1 \sigma$ & $\mathrm{K} / \mathrm{Cl}$ & $\% 1 \sigma$ & $\mathrm{Ca} / \mathrm{Cl}$ & $\% 1 \sigma$ \\
\hline 5F041P.01 & $770^{\circ} \mathrm{C}$ & 295.5 & 0 & 0.1869 & 0 & 0.000676 & 0 & 0.000034 & 0 & 0.000279 & 0 & 0.00039 & 0 & 0.01243 & 0 & 0 & 0 & 0.49 & 0 & 0 & 0 & 0 & 0 \\
\hline 5F041P. & $870^{\circ} \mathrm{C}$ & 295.5 & & 0.1869 & & 0.000676 & & 0.000034 & 0 & 0.000279 & 0 & 0.00039 & 0 & 0.01243 & 0 & 0 & 0 & 0.49 & 0 & 0 & 0 & 0 & 0 \\
\hline 5F041P.03 & $970^{\circ} \mathrm{C}$ & 295.5 & 0 & 0.1869 & 0 & 0.000676 & 0 & 0.000034 & 0 & 0.000279 & 0 & 0.00039 & 0 & 0.01243 & 0 & 0 & 0 & 0.49 & 0 & 0 & 0 & 0 & 0 \\
\hline 5F041P.04 & $1020^{\circ} \mathrm{C}$ & 295.5 & 0 & 0.1869 & 0 & 0.000676 & 0 & 0.000034 & 0 & 0.000279 & 0 & 0.00039 & 0 & 0.01243 & 0 & 0 & 0 & 0.49 & 0 & 0 & 0 & 0 & 0 \\
\hline 5F041P.05 & $1070^{\circ} \mathrm{C}$ & 295.5 & 0 & 0.1869 & 0 & 0.000676 & 0 & 0.000034 & 0 & 0.000279 & 0 & 0.00039 & 0 & 0.01243 & 0 & 0 & 0 & 0.49 & 0 & 0 & 0 & 0 & 0 \\
\hline 5F041P.06 & $1120^{\circ} \mathrm{C}$ & 295.5 & 0 & 0.1869 & 0 & 0.000676 & 0 & 0.000034 & 0 & 0.000279 & 0 & 0.00039 & 0 & 0.01243 & 0 & 0 & 0 & 0.49 & 0 & 0 & 0 & 0 & 0 \\
\hline 5F041P.07 & $1190^{\circ} \mathrm{C}$ & 295.5 & 0 & 0.1869 & 0 & 0.000676 & 0 & 0.000034 & 0 & 0.000279 & 0 & 0.00039 & 0 & 0.01243 & 0 & 0 & 0 & 0.49 & 0 & 0 & 0 & 0 & 0 \\
\hline $5 F 041 P .08$ & $1270^{\circ} \mathrm{C}$ & 295.5 & 0 & 0.1869 & 0 & 0.000676 & 0 & 0.000034 & 0 & 0.000279 & 0 & 0.00039 & 0 & 0.01243 & 0 & 0 & 0 & 0.49 & 0 & 0 & 0 & 0 & 0 \\
\hline $5 F 041 P .09$ & $1370^{\circ} \mathrm{C}$ & $\begin{array}{l}295.5 \\
2055\end{array}$ & 0 & 0.1869 & 0 & 0.000676 & 0 & 0.000034 & 0 & 0.000279 & 0 & 0.00039 & 0 & 0.01243 & 0 & 0 & 0 & 0.49 & 0 & 0 & 0 & 0 & 0 \\
\hline 5F041P.10 & $1670^{\circ} \mathrm{C}$ & 295.5 & 0 & 0.1869 & 0 & 0.000676 & 0 & 0.000034 & 0 & 0.000279 & 0 & 0.00039 & 0 & 0.01243 & 0 & 0 & 0 & 0.49 & 0 & 0 & 0 & 0 & 0 \\
\hline
\end{tabular}




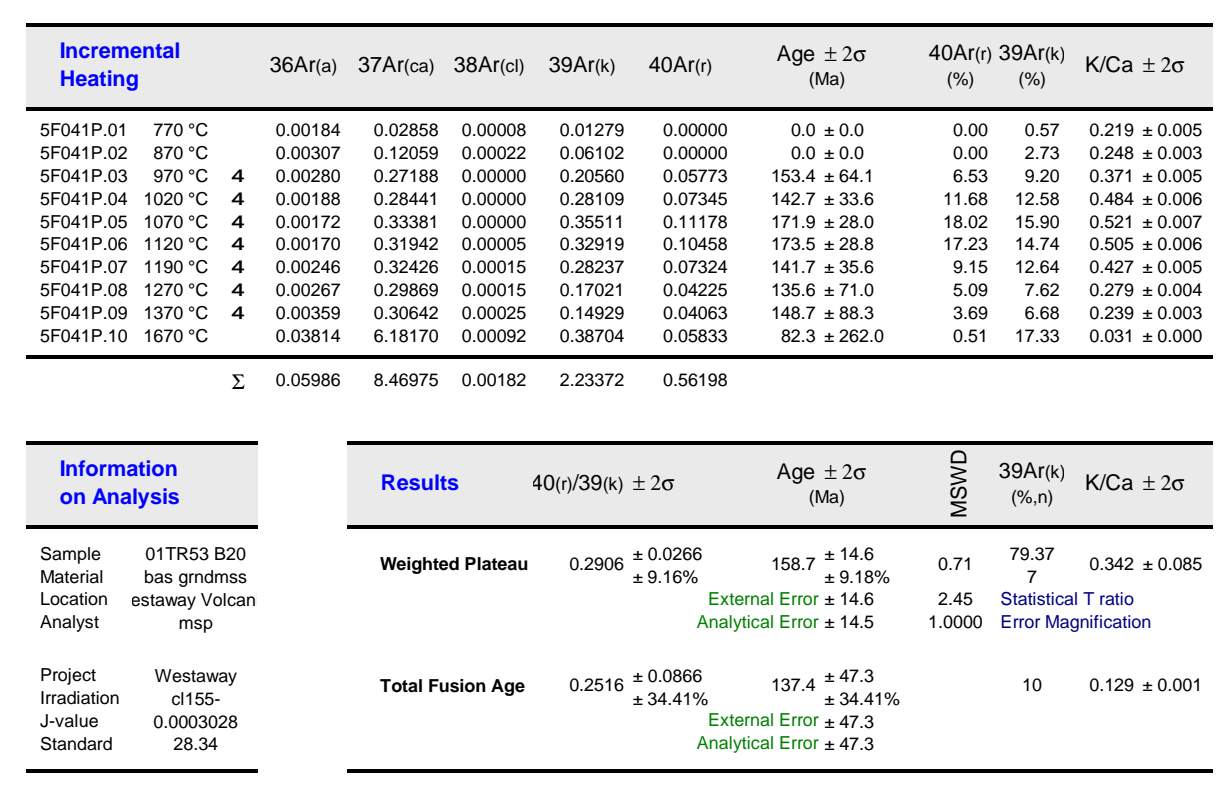




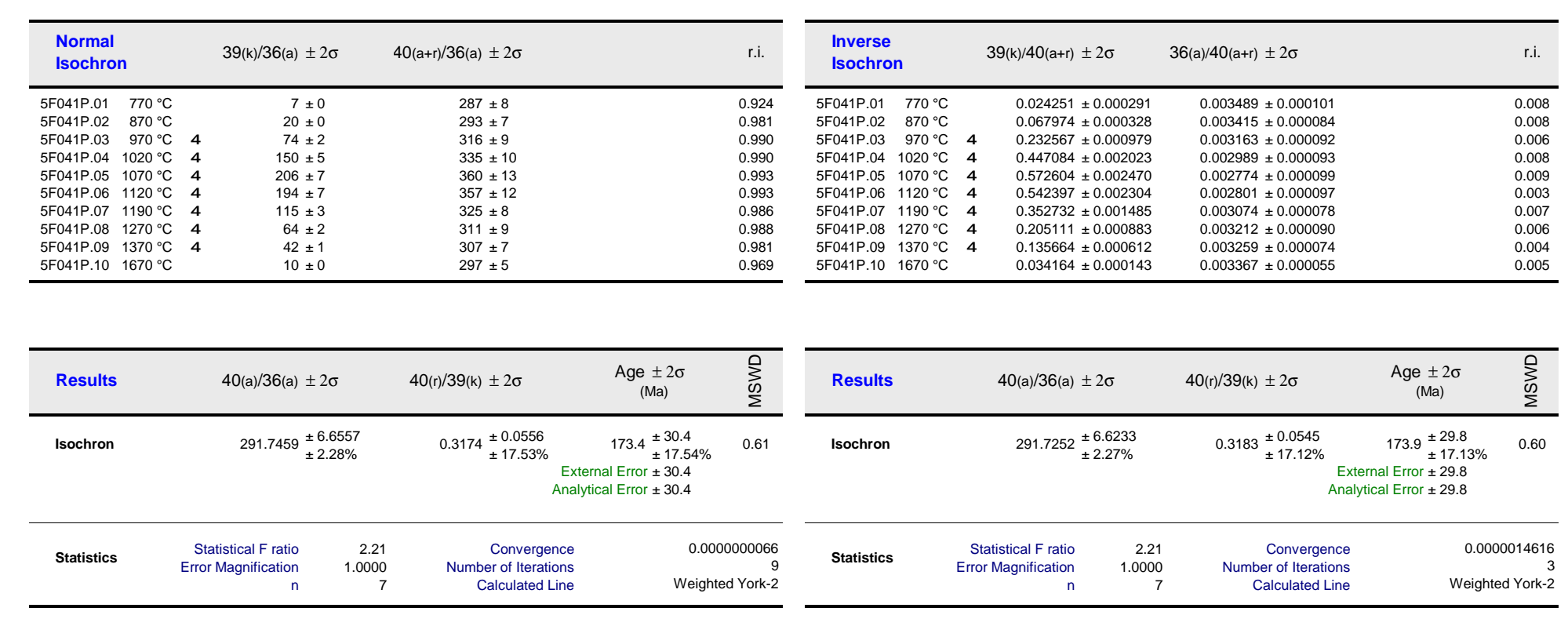




\begin{tabular}{|c|c|c|c|c|c|c|c|c|c|c|c|c|c|c|c|c|c|c|c|c|c|c|c|c|}
\hline \multicolumn{3}{|c|}{$\begin{array}{l}\text { Degassing } \\
\text { Patterns }\end{array}$} & \multirow{2}{*}{$\begin{array}{r}36 \operatorname{Ar}(\mathrm{a}) \\
0.00184\end{array}$} & \multirow{2}{*}{$\begin{array}{r}36 \operatorname{Ar}(\mathrm{ca}) \\
0.00001\end{array}$} & \multirow{2}{*}{$\begin{array}{r}36 \mathrm{Ar}(\mathrm{cl}) \\
0.00000\end{array}$} & \multirow{2}{*}{$\begin{array}{r}37 \mathrm{Ar}(\mathrm{ca}) \\
0.02858\end{array}$} & \multirow{2}{*}{$\begin{array}{l}38 \mathrm{Ar}(\mathrm{a}) \\
0.00034\end{array}$} & \multirow{2}{*}{$\begin{array}{r}38 \mathrm{Ar}(\mathrm{k}) \\
0.00016\end{array}$} & \multirow{2}{*}{$\begin{array}{r}38 \mathrm{Ar}(\mathrm{ca}) \\
0.00000\end{array}$} & \multirow{2}{*}{$\begin{array}{r}38 \mathrm{Ar}(\mathrm{cl}) \\
0.00008\end{array}$} & \multirow{2}{*}{$\begin{array}{r}39 \mathrm{Ar}(\mathrm{k}) \\
0.01279\end{array}$} & \multirow{2}{*}{$\begin{array}{r}39 \mathrm{Ar}(\mathrm{ca}) \\
0.00002\end{array}$} & \multirow{2}{*}{$\begin{array}{l}40 \operatorname{Ar}(r) \\
0.00000\end{array}$} & \multirow{2}{*}{$\begin{array}{l}40 \operatorname{Ar}(\mathrm{a}) \\
0.54383\end{array}$} & \multirow{2}{*}{$\begin{array}{r}40 \mathrm{Ar}(\mathrm{k}) \\
0.00000\end{array}$} & \multicolumn{2}{|c|}{$\begin{array}{l}\text { Additional } \\
\text { Ratios }\end{array}$} & \multirow{2}{*}{$\begin{array}{r}40(r) / 39(k) \\
0.00000\end{array}$} & \multirow{2}{*}{$\frac{1 \sigma}{0.00000}$} & \multirow{2}{*}{$\begin{array}{l}40(r+a) \\
0.52754\end{array}$} & \multirow{3}{*}{$\begin{array}{c}1 \sigma \\
0.00043 \\
0.00044\end{array}$} & \multirow{2}{*}{$\begin{array}{r}37 \mathrm{Ar} \text { (decay) } \\
4.249289\end{array}$} & \multirow{3}{*}{$\begin{array}{c}39 \mathrm{Ar} \text { (decay) } \\
1.00051696 \\
1.00051724\end{array}$} & \multirow{2}{*}{$\begin{array}{c}40 \mathrm{Ar} \text { (moles) } \\
3.165 \mathrm{E}-14\end{array}$} \\
\hline 5F041P.01 & $770^{\circ} \mathrm{C}$ & & & & & & & & & & & & & & & 5F041P.01 & $770^{\circ} \mathrm{C}$ & & & & & & & \\
\hline 5F041P.02 & $870^{\circ} \mathrm{C}$ & & 0.00307 & 0.00003 & 0.00000 & 0.12059 & 0.00057 & 0.00076 & 0.00000 & 0.00022 & 0.06102 & 0.00008 & 0.00000 & 0.90584 & 0.00002 & 5F041P.02 & $870^{\circ} \mathrm{C}$ & 0.00000 & 0.00000 & 0.89771 & & 4.252671 & & $5.386 \mathrm{E}-14$ \\
\hline 5F041P.03 & $970^{\circ} \mathrm{C}$ & 4 & 0.00280 & 0.00008 & 0.00000 & 0.27188 & 0.00052 & 0.00256 & 0.00001 & & 0.20560 & 0.00018 & 0.05773 & 0.82633 & 0.00008 & FF041P.03 & $970{ }^{\circ} \mathrm{C}$ & 0.28078 & 0.05870 & 0.88406 & 0.00039 & 4.254655 & 1.00051744 & $5.305 E-14$ \\
\hline 5F041P.04 & $1020^{\circ} \mathrm{C}$ & 4 & 0.00188 & 0.00008 & 0.00000 & 0.28441 & 0.00035 & 0.00349 & 0.00001 & 0.00000 & 0.28109 & 0.00019 & 0.07345 & 0.55528 & 0.00011 & 5F041P.04 & $1020^{\circ} \mathrm{C}$ & 0.26129 & 0.03075 & 0.62873 & 0.00033 & 4.256581 & 1.00051757 & $3.773 E-14$ \\
\hline 5F041P.05 & $1070^{\circ} \mathrm{C}$ & 4 & $\begin{array}{l}0.00172 \\
0\end{array}$ & 0.00009 & 0.00000 & 0.33381 & 0.00032 & 0.00441 & 0.00001 & 0.00000 & 0.35511 & 0.00023 & 0.11178 & 0.50838 & 0.00014 & 5F041P.05 & $1070^{\circ} \mathrm{C}$ & 0.31477 & 0.02559 & 0.62016 & 0.00037 & 4.258567 & 1.00051774 & $3.722 \mathrm{E}-14$ \\
\hline $\begin{array}{l}\text { 5F-41P.06 } \\
\text { 5F041P.07 }\end{array}$ & $\begin{array}{l}1120^{\circ} \mathrm{C} \\
1190^{\circ} \mathrm{C}\end{array}$ & $\begin{array}{l}4 \\
4\end{array}$ & $\begin{array}{l}0.00170 \\
0.00246\end{array}$ & $\begin{array}{l}0.000099 \\
0.00009\end{array}$ & $\begin{array}{l}0.000000 \\
0.00000\end{array}$ & $\begin{array}{l}0.31942 \\
0.32426\end{array}$ & $\begin{array}{l}0.00032 \\
0.00046\end{array}$ & $\begin{array}{l}0.00409 \\
0.00351\end{array}$ & $\begin{array}{l}0.000001 \\
0.00001\end{array}$ & $\begin{array}{l}0.00005 \\
0.00015\end{array}$ & $\begin{array}{l}0.32919 \\
0.28237\end{array}$ & $\begin{array}{l}0.000022 \\
0.00022\end{array}$ & $\begin{array}{l}0.10458 \\
0.07324\end{array}$ & $\begin{array}{l}0.502333 \\
0.72727\end{array}$ & $\begin{array}{l}0.00013 \\
0.00011\end{array}$ & $\begin{array}{l}\text { 5F-04P. } 06 \\
\text { 5F041P.07 }\end{array}$ & $\begin{array}{l}1120^{\circ} \mathrm{C} \\
1190{ }^{\circ} \mathrm{C}\end{array}$ & $\begin{array}{l}0.31769 \\
0.25937\end{array}$ & $\begin{array}{l}0.02634 \\
0.03260\end{array}$ & $\begin{array}{l}0.60691 \\
0.80051\end{array}$ & $\begin{array}{l}0.00019 \\
0.00035\end{array}$ & $\begin{array}{l}4.260495 \\
42648 ?\end{array}$ & $\begin{array}{l}1.00051790 \\
1.0051806\end{array}$ & $\begin{array}{l}3.642 E-14 \\
4804 E-14\end{array}$ \\
\hline $\begin{array}{l}5 F 041 P .08 \\
5 F\end{array}$ & $1270^{\circ} \mathrm{C}$ & $\begin{array}{l}4 \\
4\end{array}$ & $\begin{array}{l}0.00246 \\
0.00267\end{array}$ & 0.00008 & $\begin{array}{l}0.000000 \\
0.0000\end{array}$ & $\begin{array}{l}0.32466 \\
0.29869\end{array}$ & $\begin{array}{l}0.00466 \\
0.00050\end{array}$ & $\begin{array}{l}0.00311 \\
0.00212\end{array}$ & $\begin{array}{l}0.000001 \\
0.0001\end{array}$ & $\begin{array}{l}0.000015 \\
0.00015\end{array}$ & $\begin{array}{l}0.28231 \\
0.17021\end{array}$ & $\begin{array}{l}0.00022 \\
0.0020\end{array}$ & $\begin{array}{l}0.07324 \\
0.04225\end{array}$ & $\begin{array}{l}0.72727 \\
0.78761\end{array}$ & $\begin{array}{l}0.000007 \\
0.0007\end{array}$ & $\begin{array}{l}\text { 5F041P. } 08 \\
\text { 5F }\end{array}$ & $1270^{\circ} \mathrm{C}$ & $\begin{array}{l}0.25397 \\
0.24819\end{array}$ & $\begin{array}{l}0.03260 \\
0.06500\end{array}$ & $\begin{array}{l}0.80591 \\
0.82986\end{array}$ & $\begin{array}{l}0.00035 \\
0.0036\end{array}$ & $\begin{array}{l}4.262482 \\
4.264470\end{array}$ & $\begin{array}{l}1.000051806 \\
1.00051823\end{array}$ & $\begin{array}{l}4.804 \mathrm{E}-14 \\
4.980 \mathrm{E}-14\end{array}$ \\
\hline \multirow{3}{*}{$\begin{array}{l}\text { 5F041P.09 } \\
\text { 5F041P.10 }\end{array}$} & $\begin{array}{l}1370^{\circ} \mathrm{C} \\
1670^{\circ} \mathrm{C}\end{array}$ & 4 & $\begin{array}{l}0.00359 \\
0.03814\end{array}$ & $\begin{array}{l}0.00009 \\
0.00172\end{array}$ & $\begin{array}{l}0.00000 \\
0.00000\end{array}$ & $\begin{array}{l}0.30642 \\
618180\end{array}$ & $\begin{array}{l}0.00067 \\
0.00713\end{array}$ & $\begin{array}{l}0.00186 \\
0.0048\end{array}$ & $\begin{array}{l}0.000011 \\
0.00021\end{array}$ & $\begin{array}{l}0.00025 \\
0.00092\end{array}$ & $\begin{array}{l}0.14929 \\
0.38704\end{array}$ & $\begin{array}{l}0.00021 \\
0.0048\end{array}$ & $\begin{array}{l}0.04063 \\
0.05833\end{array}$ & $\begin{array}{r}1.05984 \\
1127050\end{array}$ & $\begin{array}{l}0.00006 \\
0.00015\end{array}$ & $\begin{array}{l}\text { 5F04P.09 } \\
\text { 5F04P.10 }\end{array}$ & $\begin{array}{l}1370^{\circ} \mathrm{C} \\
1670^{\circ} \mathrm{C}\end{array}$ & $\begin{array}{l}0.27216 \\
0.15071\end{array}$ & $\begin{array}{l}0.08085 \\
0.23984\end{array}$ & $\begin{array}{r}1.10047 \\
1132883\end{array}$ & $\begin{array}{l}0.00035 \\
0.00341\end{array}$ & $\begin{array}{l}4.266401 \\
4268391\end{array}$ & $\begin{array}{l}1.00051839 \\
1.00051856\end{array}$ & $\begin{array}{l}6.603 E-14 \\
6797 E-13\end{array}$ \\
\hline & & & 005986 & & م000000 & 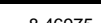 & 001119 & 002777 & م00029 & 000182 & 203379 & & & & & & & & & & & & & \\
\hline & & & & & & 16075 & & & & & & & & & & & & & & & & & & \\
\hline
\end{tabular}


Age Plateau 


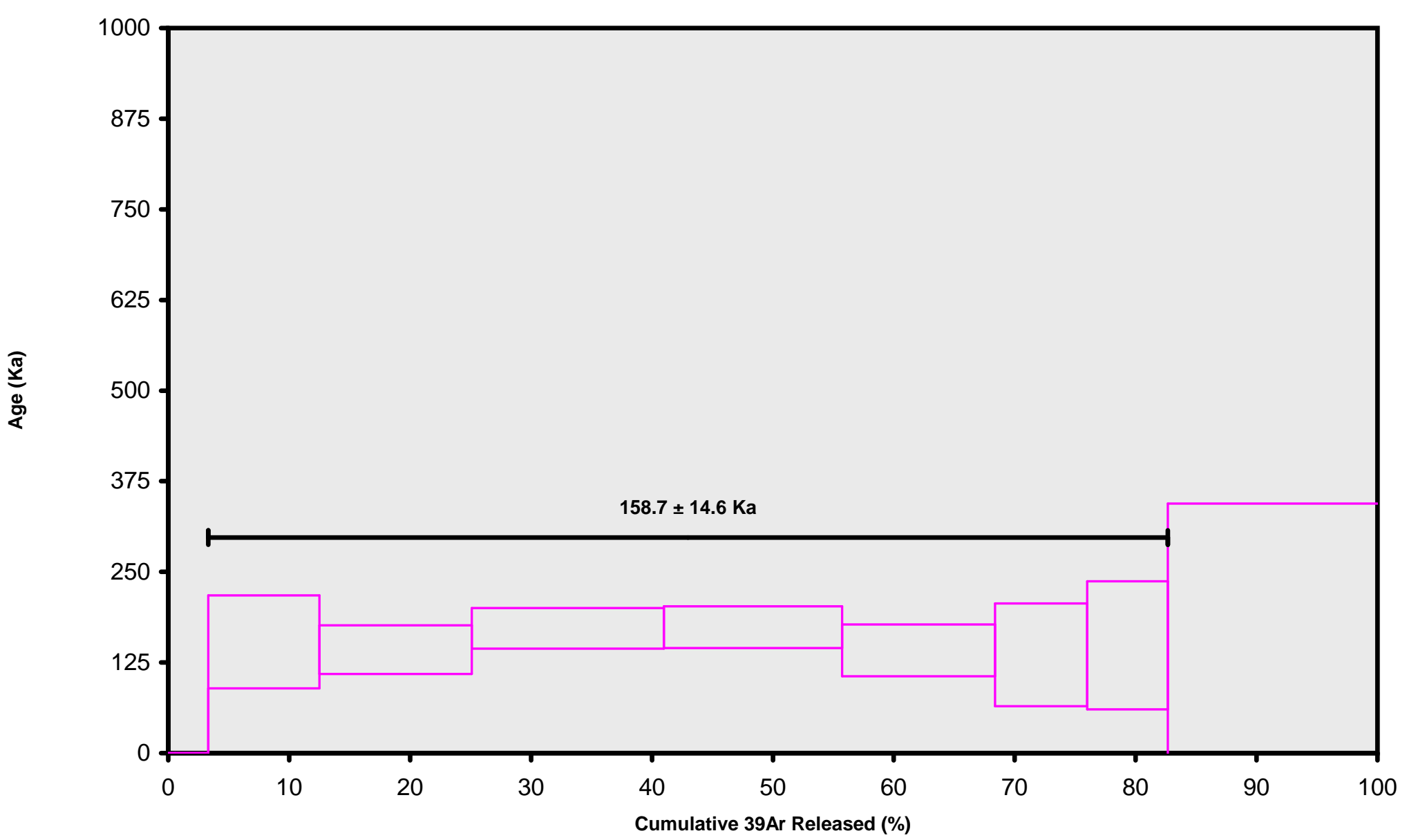

\section{Ar-Ages in $\mathrm{Ka}$}

Weighted Plateau

$158.7 \pm 14.6$

Total Fusion

$137.4 \pm 47.3$

Normal Isochron

$173.4 \pm 30.4$

Inverse Isochron

$173.9 \pm 29.8$

\section{MSWD}

0.71

Sample Info
bas grndmss
Westaway Volcanics
msp
cl155-
$0.0003028(\mathrm{~J})$


K-Ca Plateau 


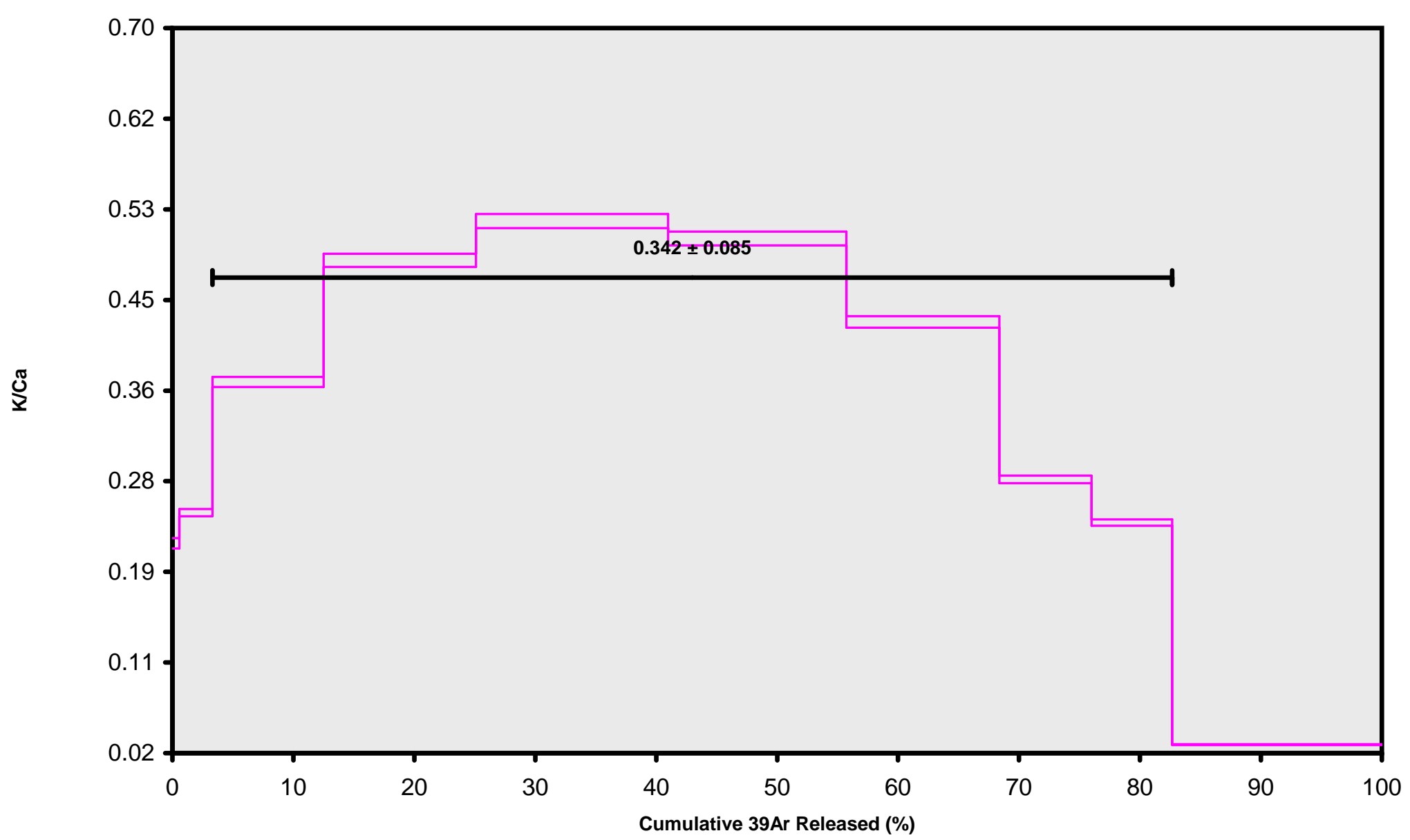

\section{Ar-Ages in $\mathrm{Ka}$}

Weighted Plateau

$158.7 \pm 14.6$

Total Fusion

$137.4 \pm 47.3$

Normal Isochron

$173.4 \pm 30.4$

Inverse Isochron

$173.9 \pm 29.8$

Sample Info

bas grndmss

Westaway Volcanics

$\mathrm{msp}$

cl155-

$0.0003028(\mathrm{~J})$ 
Normal Isochron 


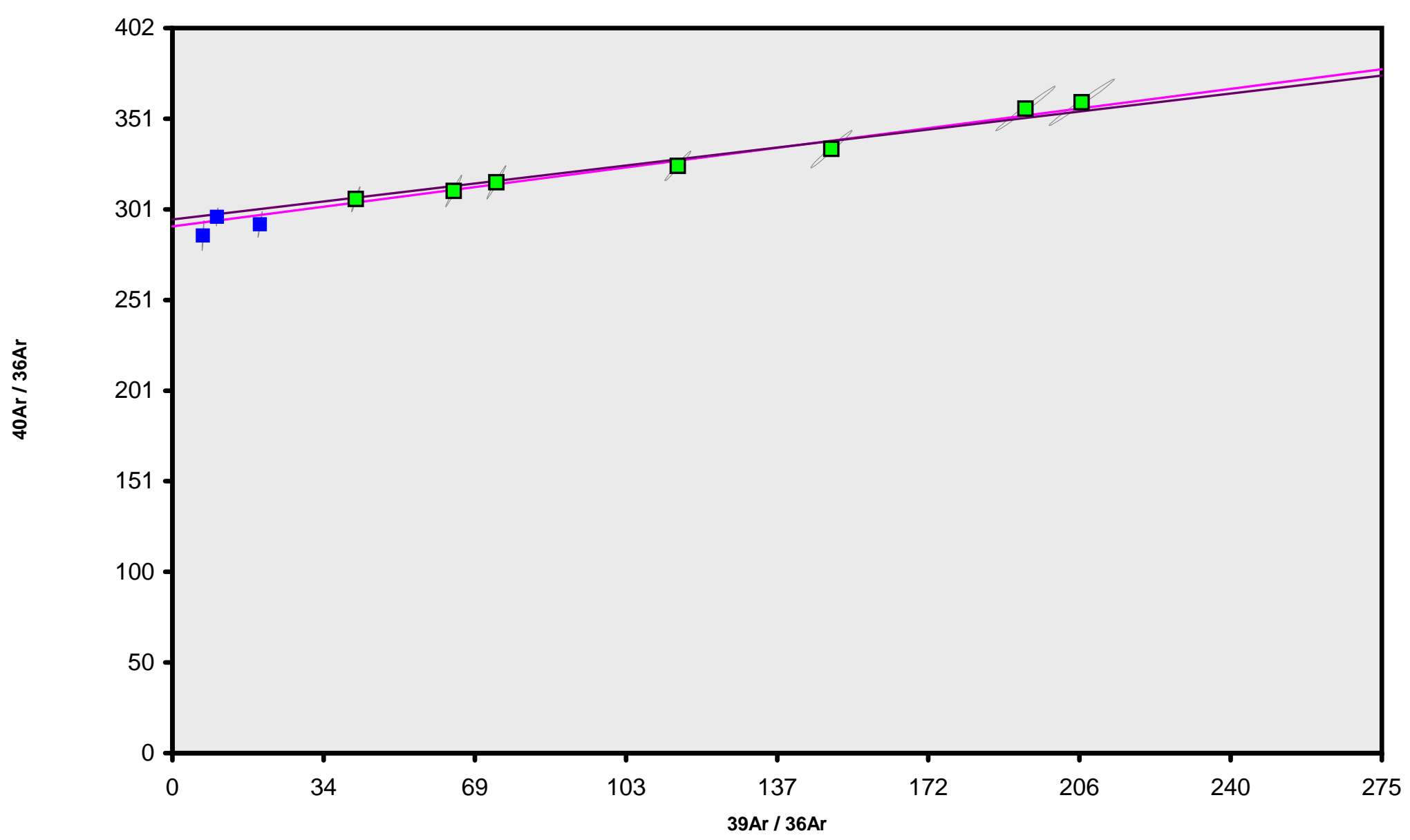

Ar-Ages in $\mathrm{Ka}$

Weighted Plateau

$158.7 \pm 14.6$

Total Fusion

$137.4 \pm 47.3$

Normal Isochron

$173.4 \pm 30.4$

Inverse Isochron

$173.9 \pm 29.8$

\section{MSWD}

0.61

Sample Info

bas grndmss

Westaway Volcanics

$\mathrm{msp}$

cl155-

$0.0003028(\mathrm{~J})$ 


\section{Inverse Isochron}




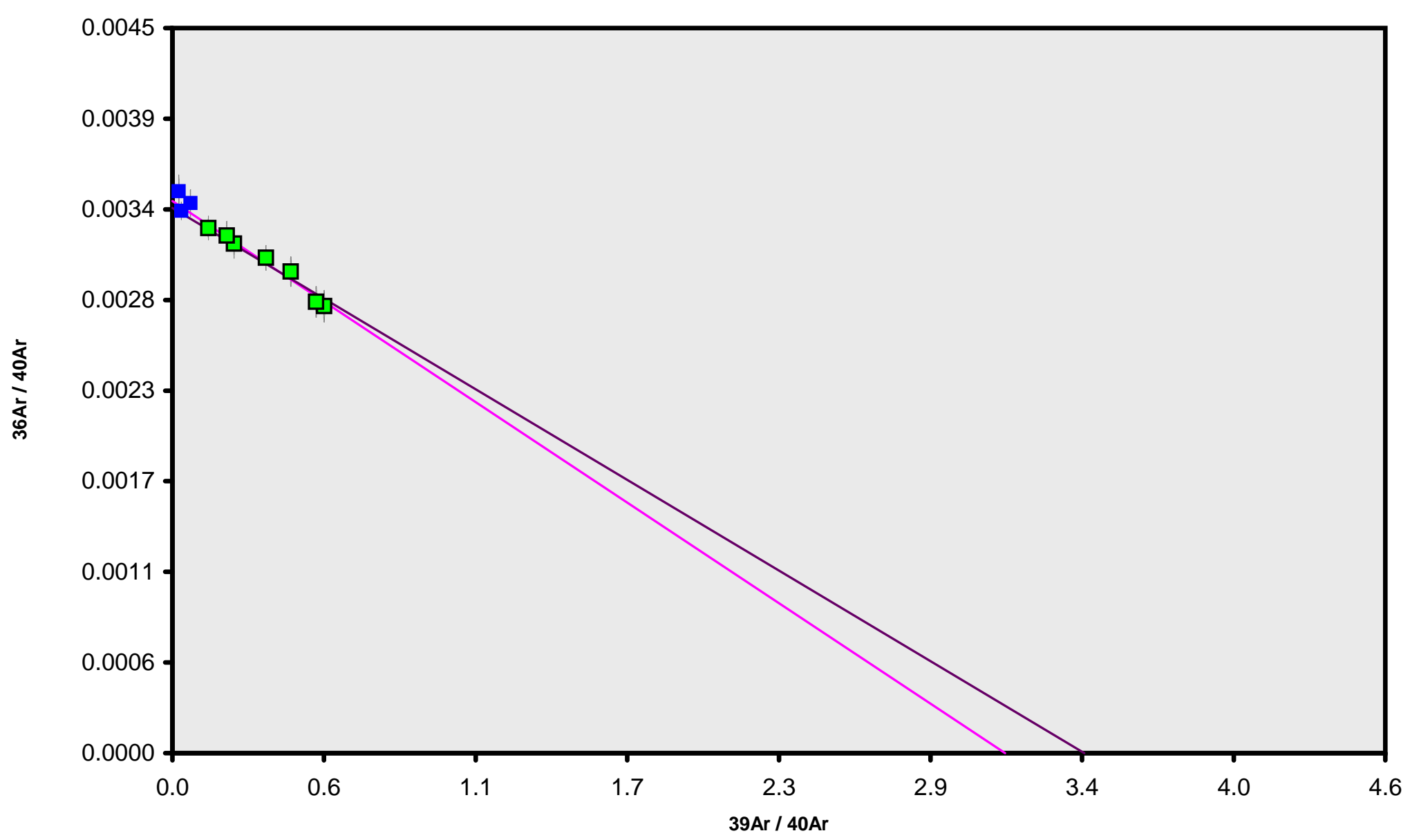

\section{Ar-Ages in $\mathrm{Ka}$}

Weighted Plateau

$158.7 \pm 14.6$

Total Fusion

$137.4 \pm 47.3$

Normal Isochron

$173.4 \pm 30.4$

Inverse Isochron

$173.9 \pm 29.8$

\section{MSWD}

0.60

Sample Info

bas grndmss

Westaway Volcanics

msp

cl155-

$0.0003028(\mathrm{~J})$ 
Sample 03TR21 
Data Tables 
MIT, Cambridge, USA

\begin{tabular}{|c|c|c|c|c|c|c|c|c|c|c|c|}
\hline \multicolumn{2}{|c|}{$\begin{array}{l}\text { Procedure } \\
\text { Blanks }\end{array}$} & $36 \mathrm{Ar}$ & $1 \sigma$ & $37 \mathrm{Ar}$ & $1 \sigma$ & $38 \mathrm{Ar}$ & $1 \sigma$ & $39 \mathrm{Ar}$ & $1 \sigma$ & $40 \mathrm{Ar}$ & $1 \sigma$ \\
\hline 5F041M.01 & & 0.000230 & 0.000017 & 0.000207 & 0.000034 & 0.000127 & 0.000024 & 0.000394 & 0.000038 & 0.040000 & 0.000122 \\
\hline $\begin{array}{l}5 \mathrm{~F} 041 \mathrm{M} .02 \\
5 \mathrm{5} F 41 \mathrm{M}\end{array}$ & $\begin{array}{l}870^{\circ} \mathrm{C} \\
970^{\circ} \mathrm{C}\end{array}$ & 0.000231 & $\begin{array}{l}0.000017 \\
0.000017\end{array}$ & $\begin{array}{l}0.000209 \\
0.00211\end{array}$ & $\begin{array}{l}0.000034 \\
0.000034\end{array}$ & $\begin{array}{l}0.000127 \\
0.00127\end{array}$ & 0.000024 & 0.000396 & 0.000038 & 0.040000 & 0.000122 \\
\hline 5F041M.04 & $1020^{\circ} \mathrm{C}$ & 0.000231 & $\begin{array}{l}0.000017 \\
0.000017\end{array}$ & $\begin{array}{l}0.000211 \\
0.000213\end{array}$ & $\begin{array}{l}0.000034 \\
0.000034\end{array}$ & $\begin{array}{l}0.000127 \\
0.000128\end{array}$ & $\begin{array}{l}0.000024 \\
0.000024\end{array}$ & $\begin{array}{l}0.000397 \\
0.000398\end{array}$ & $\begin{array}{l}0.000038 \\
0.000038\end{array}$ & $\begin{array}{l}0.0 .0400000 \\
0.040000\end{array}$ & $\begin{array}{l}0.000122 \\
0.000122\end{array}$ \\
\hline 5F041M.05 & $1070^{\circ} \mathrm{C}$ & 0.000232 & 0.000017 & 0.000215 & 0.000034 & 0.000128 & 0.000024 & 0.000399 & 0.000038 & 0.040000 & 0.000122 \\
\hline 5F041M.06 & $1120^{\circ} \mathrm{C}$ & 0.000232 & 0.000017 & 0.000216 & 0.000034 & 0.000128 & 0.000024 & 0.000400 & 0.000038 & 0.040000 & 0.000122 \\
\hline 5F041M.07 & $1190^{\circ} \mathrm{C}$ & 0.000232 & 0.000017 & 0.000218 & 0.000034 & 0.000129 & 0.000024 & 0.000401 & 0.000038 & & 0.000122 \\
\hline 5F041M.08 & $1270^{\circ} \mathrm{C}$ & 0.000233 & 0.000017 & 0.000220 & 0.000034 & 0.000129 & 0.000024 & 0.000403 & 0.000038 & 0.040000 & 0.000122 \\
\hline $\begin{array}{l}5 \text { FF41M.09 } \\
\text { 5041M.10 }\end{array}$ & $\begin{array}{l}3370^{\circ} \mathrm{C} \\
167{ }^{\circ} \mathrm{C}\end{array}$ & $\begin{array}{l}0.000233 \\
0.000233\end{array}$ & $\begin{array}{l}0.000017 \\
0.000017\end{array}$ & $\begin{array}{l}0.000222 \\
0.000224\end{array}$ & $\begin{array}{l}0.000034 \\
0.000034\end{array}$ & $\begin{array}{l}0.000129 \\
0.000130\end{array}$ & $\begin{array}{l}0.000024 \\
0.000024\end{array}$ & $\begin{array}{l}0.00040404 \\
0.0004405\end{array}$ & $\begin{array}{l}0.000038 \\
0.000038\end{array}$ & $\begin{array}{l}0.04400000 \\
0.040000\end{array}$ & $\begin{array}{l}0.000122 \\
0.000122\end{array}$ \\
\hline
\end{tabular}




\begin{tabular}{|c|c|c|c|c|c|c|c|c|c|c|c|c|c|c|c|c|c|c|c|c|}
\hline $\begin{array}{l}\text { Intercep } \\
\text { Values }\end{array}$ & & $36 \mathrm{Ar}$ & $1 \sigma$ & r2 & & $37 \mathrm{Ar}$ & $1 \sigma$ & r2 & & $38 \mathrm{Ar}$ & $1 \sigma$ & $\mathrm{r} 2$ & & $39 \mathrm{Ar}$ & $1 \sigma$ & r2 & & $40 \mathrm{Ar}$ & $1 \sigma$ & $r^{2}$ \\
\hline $\begin{array}{l}\text { 5F041M.01 } \\
\text { 5F041M.02 }\end{array}$ & $\begin{array}{l}770^{\circ} \mathrm{C} \\
870^{\circ} \mathrm{C}\end{array}$ & $\begin{array}{l}0.002041 \\
0.002803\end{array}$ & $\begin{array}{l}0.000022 \\
0.000029\end{array}$ & $\begin{array}{l}0.5804 \\
0.7290\end{array}$ & $\begin{array}{l}\operatorname{LIN} \# 1 \\
\operatorname{IN} \#\end{array}$ & $\begin{array}{l}0.007738 \\
0.036700\end{array}$ & $\begin{array}{l}0.000015 \\
0.000039\end{array}$ & $\begin{array}{l}0.0864 \\
0.9978\end{array}$ & $\begin{array}{l}\operatorname{LIN} \# 1215 \\
\text { EXP \#15 }\end{array}$ & 0.000643 & 0.000034 & 0.0106 & LIN \#1 & 0.007625 & 0.000042 & 0.9951 & EXP \#1 15 & 0.558655 & 0.000593 & 0.9837 EXP \#124911 \\
\hline $\begin{array}{l}5 \mathrm{~F} 041 \mathrm{M} .03 \\
5\end{array}$ & $970^{\circ} \mathrm{C}$ & 0.002307 & $\begin{array}{l}0.0000012 \\
0.00029\end{array}$ & $\begin{array}{l}0.7230 \\
0.8366\end{array}$ & $\operatorname{LN} \# 1$ & 0.091589 & 0.000095 & 0.9986 & $\begin{array}{l}\operatorname{EXP} \# 15 \\
\operatorname{EXP} \# 1691115\end{array}$ & 0.003054 & 0.000030 & $\begin{array}{l}0.7134 \\
0.7234\end{array}$ & LIN \#115 & $\begin{array}{l}0.049973 \\
0.209897\end{array}$ & $\begin{array}{l}0.000214 \\
0.000\end{array}$ & $\begin{array}{l}0.9934 \\
0.9972\end{array}$ & EXP\# & $\begin{array}{l}0.741652 \\
0.7452\end{array}$ & 0.000366 & $\begin{array}{l}0.0800 \\
0.9744 \text { EXP \# \#1 } 114\end{array}$ \\
\hline 5F041M.04 & $1020^{\circ} \mathrm{C}$ & 0.001719 & 0.000023 & 0.1339 & LIN \# 1 & 0.111823 & 0.000093 & 0.9989 & EXP\#18 & 0.004015 & 0.000032 & 0.8470 & LIN \# 115 & 0.297452 & 0.000272 & 0.9982 & EXP \# 1 & 0.628357 & 0.000282 & 0.9978 EXP \# 1610 \\
\hline 5F041M.06 & $1120^{\circ} \mathrm{C}$ & 0.001431 & 0.000018 & 0.0035 & LIN \#1 13 & 0.116670 & 0.000129 & 0.9977 & LIN \# 11 & 0.004110 & 0.000025 & 0.8904 & LIN \# 113 & 0.306541 & 0.000156 & 0.9995 & 5 EXP \# 1 & 0.558668 & 0.000282 & 0.9988 EXP \# 167 \\
\hline 5F041M.07 & $1190^{\circ} \mathrm{C}$ & 0.001490 & 0.000021 & 0.0026 & LIN \# & 0.074359 & 0.000074 & 0.9977 & EXP \# & 0.003603 & 0.000033 & 0.8175 & LIN \# 15 & 0.260721 & 0.000146 & 0.9993 & EXP\# 1 & 0.553057 & 0.000154 & 0.9995 EXP \# 1 \\
\hline 5F041M.08 & $\begin{array}{l}1270^{\circ} \mathrm{C} \\
1370^{\circ} \mathrm{C}\end{array}$ & 0.001689 & 0.000020 & 0.0908 & LIN \# & 0.057733 & 0.000067 & 0.9971 & $\begin{array}{l}\operatorname{LIN} \# 1 \\
\text { EXP \# }\end{array}$ & 0.002991 & $\begin{array}{l}0.000015 \\
\end{array}$ & 0.9086 & LIN \# 11115 & 0.205999 & 0.000214 & 0.9976 & EXP \# 110 & 0.575979 & 0.000349 & 0.9971 EXP \# 1 5 \\
\hline $\begin{array}{l}\text { 5FF041M.09 } \\
\text { 5F041M.10 }\end{array}$ & $\begin{array}{l}1370^{\circ} \mathrm{C} \\
1670^{\circ} \mathrm{C}\end{array}$ & $\begin{array}{l}0.001334 \\
0.007130\end{array}$ & $\begin{array}{l}0.000022 \\
0.000016\end{array}$ & 0.02833 & $\begin{array}{l}\operatorname{LiN} \# \\
\text { EXP \#913 }\end{array}$ & $\begin{array}{l}0.055524 \\
0.926481\end{array}$ & $\begin{array}{l}0.0000084 \\
0.000270\end{array}$ & $\begin{array}{l}0.9950 \\
0.9999\end{array}$ & EXP\#1 & $\begin{array}{l}0.000542 \\
0.006026\end{array}$ & $\begin{array}{l}0.000022 \\
0.000040\end{array}$ & $\begin{array}{l}0.6421 \\
0.8442\end{array}$ & $\begin{array}{l}\operatorname{LN} \# \\
\operatorname{LIN} \# 1\end{array}$ & $\begin{array}{l}0.174835 \\
0.369766\end{array}$ & $\begin{array}{l}0.000121 \\
0.000230\end{array}$ & $\begin{array}{l}0.9990 \\
0.9992\end{array}$ & EXP \#1111 & $\begin{array}{l}0.455636 \\
1.995365\end{array}$ & $\begin{array}{l}0.000287 \\
0.000494\end{array}$ & 0.9990 EXP\# \#179 15 \\
\hline
\end{tabular}




\begin{tabular}{|c|c|c|c|c|c|c|c|c|c|c|c|c|c|c|c|c|c|c|c|c|c|c|}
\hline $\begin{array}{l}\text { Sample } \\
\text { Parameters }\end{array}$ & Sample & Material & Location & Analyst & 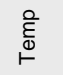 & $\begin{array}{c}\text { Standard } \\
\text { (in Ma) }\end{array}$ & $\% 1 \sigma$ & $\mathrm{J}$ & $\% 1 \sigma$ & Fract & $\% 1 \sigma$ & $\begin{array}{l}\text { Volume } \\
\text { Corr. }\end{array}$ & $\begin{array}{c}\text { Sensitivity } \\
\text { (mol/vol) }\end{array}$ & बึ & $\begin{array}{l}\text { 訾 } \\
\text { 立 }\end{array}$ & 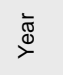 & 호 & $\frac{c}{\Sigma}$ & 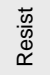 & Irradiation & Project & $\begin{array}{c}\text { Standard } \\
\text { Name }\end{array}$ \\
\hline $\begin{array}{l}\text { 5F041M.01 } 770^{\circ} \mathrm{C} \\
\text { 5F041M.02 } \\
870^{\circ} \mathrm{C}\end{array}$ & $\begin{array}{l}\text { 01TR21 B17 } \\
01 \text { TR21 B17 }\end{array}$ & $\begin{array}{l}\text { bas grndmss } \\
\text { bas grndmss }\end{array}$ & $\begin{array}{l}\text { Westaway } \\
\text { Westaway }\end{array}$ & $\begin{array}{l}\mathrm{msp} \\
\mathrm{msp}\end{array}$ & $\begin{array}{l}770 \\
870\end{array}$ & $\begin{array}{l}28.34 \\
28.34\end{array}$ & $\begin{array}{l}0.01 \\
0.01\end{array}$ & $\begin{array}{l}0.0003142 \\
0.0003142\end{array}$ & $\begin{array}{l}0.3 \\
0.3\end{array}$ & $\begin{array}{l}1.007 \\
1.007\end{array}$ & $\begin{array}{l}0.2 \\
0.2\end{array}$ & $\begin{array}{l}1 \\
1\end{array}$ & $\begin{array}{l}6.000 \mathrm{E}-14 \\
6.000 \mathrm{E}-14\end{array}$ & $\begin{array}{l}29 \\
29\end{array}$ & $\begin{array}{l}07 \\
07\end{array}$ & $\begin{array}{l}2005 \\
2005\end{array}$ & $\begin{array}{l}03 \\
03\end{array}$ & $\begin{array}{l}00 \\
58\end{array}$ & $\begin{array}{l}001 \\
001\end{array}$ & $\begin{array}{l}\text { cl155- } \\
\text { cl155- }\end{array}$ & $\begin{array}{l}\text { Westaway } \\
\text { Westaway }\end{array}$ & $\begin{array}{l}\text { tcr-2a } \\
\text { tcr-2a }\end{array}$ \\
\hline 5F041M.03 $970^{\circ} \mathrm{C}$ & 01 TR21 B17 & bas grndmss & Westaway & $\mathrm{msp}$ & 970 & 28.34 & 0.01 & 0.0003142 & 0.3 & 1.007 & 0.2 & 1 & $6.000 E-14$ & 29 & 07 & 2005 & 04 & 31 & 001 & cl155- & Westaway & tcr-2a \\
\hline 5F041M.04 $10200^{\circ} \mathrm{C}$ & 01TR21 B17 & bas grndmss & Westaway & msp & 1020 & 28.34 & 0.01 & 0.0003142 & 0.3 & 1.007 & 0.2 & 1 & $6.000 E-14$ & 29 & 07 & 2005 & 05 & 05 & 001 & cl155- & Westaway & tcr-2a \\
\hline 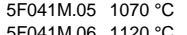 & 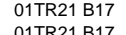 & bas grndmss & Westaway & $\mathrm{msp}$ & 1070 & 28.34 & 0.01 & & 0.3 & 1.007 & 0.2 & 1 & $6.000 E-14$ & 29 & 07 & 2005 & 05 & 39 & 001 & & Westaway & tcr-2a \\
\hline $\begin{array}{l}5 \times 5411.06 \quad 1120^{\circ} \mathrm{C} \\
50541107.119{ }^{\circ} \mathrm{C}\end{array}$ & 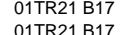 & $\begin{array}{l}\text { bas grndmss } \\
\text { bas arndmss }\end{array}$ & $\begin{array}{l}\text { Westaway } \\
\text { Westazay }\end{array}$ & $\mathrm{msp}$ & 1120 & 28.34 & 0.01 & 0.0003142 & 0.3 & 1.007 & 0.2 & 1 & $6.000 E-14$ & 29 & 07 & 2005 & 06 & 12 & 001 & cl155- & Westaway & tcr-2a \\
\hline 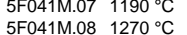 & 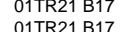 & bas grndmss & $\begin{array}{l}\text { Westaway } \\
\text { Westanay }\end{array}$ & msp & $\begin{array}{l}1190 \\
11970\end{array}$ & $\begin{array}{l}28.34 \\
2834\end{array}$ & 0.01 & 0.0003142 & 0.3 & $\begin{array}{l}1.007 \\
1.007\end{array}$ & 0.2 & 1 & $6.000 E-14$ & 29 & 07 & 2005 & 06 & 46 & 001 & cl155- & Westaway & tcr-2a \\
\hline $5 F 041 \mathrm{M} .091370^{\circ} \mathrm{C}$ & $\begin{array}{l}01 \text { TR21 B17 } \\
\text { B }\end{array}$ & $\begin{array}{l}\text { Das ginnamis } \\
\text { bas grndmss }\end{array}$ & $\begin{array}{l}\text { Westaway } \\
\text { Westay }\end{array}$ & $\begin{array}{l}\text { msp } \\
\text { msp }\end{array}$ & 1370 & $\begin{array}{l}28.34 \\
28.34\end{array}$ & 0.01 & $\begin{array}{l}0.00003142 \\
0.002\end{array}$ & $\begin{array}{l}0.3 \\
0.3\end{array}$ & $\begin{array}{l}1.007 \\
1.007\end{array}$ & 0.2 & 1 & $\begin{array}{l}6.000 E-14 \\
6.000\end{array}$ & 29 & 07 & 2005 & 07 & $\begin{array}{l}19 \\
53\end{array}$ & 001 & $\begin{array}{l}\text { cl155- } \\
\text { cliss- }\end{array}$ & $\begin{array}{l}\text { Westaway } \\
\text { Westay }\end{array}$ & $\begin{array}{l}\text { Tct-2a } \\
\text { tcr-2a }\end{array}$ \\
\hline 5F041M.10 $1670^{\circ} \mathrm{C}$ & 01TR21 B17 & bas grndmss & Westaway & msp & 1670 & 28.34 & 0.01 & 0.0003142 & 0.3 & 1.007 & 0.2 & 1 & $6.000 E-14$ & 29 & 07 & 2005 & 08 & 26 & 001 & cl155- & Westaway & tcr-2a \\
\hline
\end{tabular}


MIT, Cambridge, USA

\begin{tabular}{|c|c|c|c|c|c|c|c|c|c|c|c|c|c|c|c|c|c|c|c|c|c|c|c|}
\hline $\begin{array}{l}\text { Irradiatio } \\
\text { Constan }\end{array}$ & & 40/36(a) & $\% 1 \sigma$ & 38/36(a) & $\% 1 \sigma$ & 39/37(ca) & $\% 1 \sigma$ & $38 / 37$ (ca) & $\% 1 \sigma$ & $36 / 37$ (ca) & $\% 1 \sigma$ & $40 / 39(k)$ & $\% 1 \sigma$ & $38 / 39(\mathrm{k})$ & $\% 1 \sigma$ & 36/38(cl) & $\% 1 \sigma$ & $\mathrm{K} / \mathrm{Ca}$ & $\% 1 \sigma$ & $\mathrm{K} / \mathrm{Cl}$ & $\% 1 \sigma$ & $\mathrm{Ca} / \mathrm{Cl}$ & $\% 1 \sigma$ \\
\hline 5F041M.01 & $770^{\circ} \mathrm{C}$ & $\begin{array}{l}295.5 \\
2055\end{array}$ & 0 & 0.1869 & 0 & 0.000676 & 0 & 0.000034 & 0 & 0.000279 & 0 & 0.00039 & 0 & 0.01243 & 0 & 0 & 0 & 0.49 & 0 & 0 & 0 & 0 & 0 \\
\hline 5F041M.02 & $870^{\circ} \mathrm{C}$ & $\begin{array}{l}295.5 \\
295.5\end{array}$ & 0 & 0.1869 & 0 & 0.000676 & 0 & 0.000034 & 0 & 0.000279 & 0 & 0.00039 & 0 & 0.01243 & 0 & 0 & 0 & 0.49 & 0 & 0 & 0 & 0 & 0 \\
\hline 5F041M.03 & 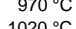 & $\begin{array}{l}295.5 \\
2955\end{array}$ & 0 & $\begin{array}{l}0.1869 \\
0.1869\end{array}$ & 0 & $\begin{array}{l}0.000676 \\
0.00667\end{array}$ & 0 & $\begin{array}{l}0.000034 \\
0.000034\end{array}$ & $\begin{array}{l}0 \\
0 \\
0\end{array}$ & $\begin{array}{l}0.000279 \\
0.00279\end{array}$ & 0 & $\begin{array}{l}0.00039 \\
0.00039\end{array}$ & $\begin{array}{l}0 \\
0 \\
0\end{array}$ & $\begin{array}{l}0.01243 \\
0.01243\end{array}$ & 0 & 0 & 0 & $\begin{array}{l}0.49 \\
0.49\end{array}$ & 0 & 0 & 0 & $\begin{array}{l}0 \\
0 \\
0\end{array}$ & 0 \\
\hline 5F041M.04 & $\begin{array}{l}1020^{\circ} \mathrm{C} \\
1070^{\circ} \mathrm{C}\end{array}$ & $\begin{array}{l}295.5 \\
295.5\end{array}$ & $\begin{array}{l}0 \\
0\end{array}$ & $\begin{array}{l}0.1869 \\
0.1869\end{array}$ & $\begin{array}{l}0 \\
0 \\
0\end{array}$ & 0.000676 & $\begin{array}{l}0 \\
0\end{array}$ & $\begin{array}{l}0.000034 \\
0.000034\end{array}$ & $\begin{array}{l}0 \\
0\end{array}$ & $\begin{array}{l}0.000279 \\
0.00279\end{array}$ & $\begin{array}{l}0 \\
0 \\
0\end{array}$ & $\begin{array}{l}0.000039 \\
0.00039\end{array}$ & $\begin{array}{l}0 \\
0 \\
0\end{array}$ & $\begin{array}{l}0.01243 \\
0.01243\end{array}$ & $\begin{array}{l}0 \\
0\end{array}$ & 0 & 0 & $\begin{array}{l}0.49 \\
0.49\end{array}$ & 0 & 0 & 0 & $\begin{array}{l}0 \\
0\end{array}$ & 0 \\
\hline 5F041M.05 & $\begin{array}{l}10700^{\circ} \mathrm{C} \\
1120^{\circ}\end{array}$ & 295.5 & 0 & 0.1869 & 0 & 0.000676 & 0 & 0.000034 & 0 & 0.000279 & 0 & 0.00039 & 0 & 0.01243 & 0 & 0 & 0 & $\begin{array}{l}0.49 \\
0.49\end{array}$ & 0 & 0 & 0 & 0 & $\begin{array}{l}0 \\
0 \\
0\end{array}$ \\
\hline 5F041M.07 & $1190^{\circ} \mathrm{C}$ & 295.5 & 0 & 0.1869 & 0 & 0.000676 & 0 & 0.000034 & 0 & 0.000279 & 0 & 0.00039 & 0 & 0.01243 & 0 & 0 & 0 & 0.49 & 0 & 0 & 0 & 0 & 0 \\
\hline 5F041M.08 & $1270^{\circ} \mathrm{C}$ & 295.5 & 0 & 0.1869 & 0 & 0.000676 & 0 & 0.000034 & 0 & 0.000279 & 0 & 0.00039 & 0 & 0.01243 & 0 & 0 & 0 & 0.49 & 0 & 0 & 0 & 0 & 0 \\
\hline 5F041M.09 & $1370^{\circ} \mathrm{C}$ & 295.5 & 0 & 0.1869 & 0 & 0.000676 & 0 & 0.000034 & 0 & 0.000279 & 0 & 0.00039 & 0 & 0.01243 & 0 & 0 & 0 & 0.49 & 0 & 0 & 0 & 0 & 0 \\
\hline 5F041M.10 & $1670^{\circ} \mathrm{C}$ & 295.5 & 0 & 0.1869 & 0 & 0.000676 & 0 & 0.000034 & 0 & 0.000279 & 0 & 0.00039 & 0 & 0.01243 & 0 & 0 & 0 & 0.49 & 0 & 0 & 0 & 0 & 0 \\
\hline
\end{tabular}




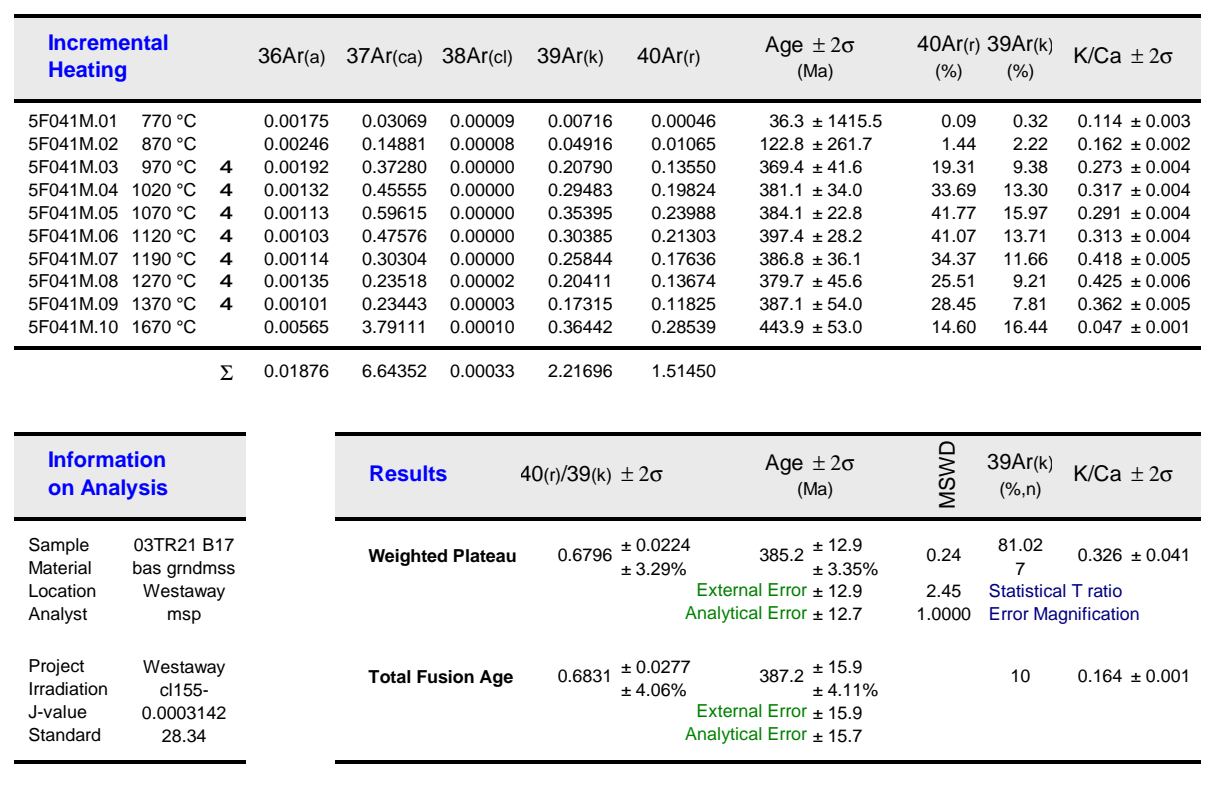


MIT, Cambridge, USA

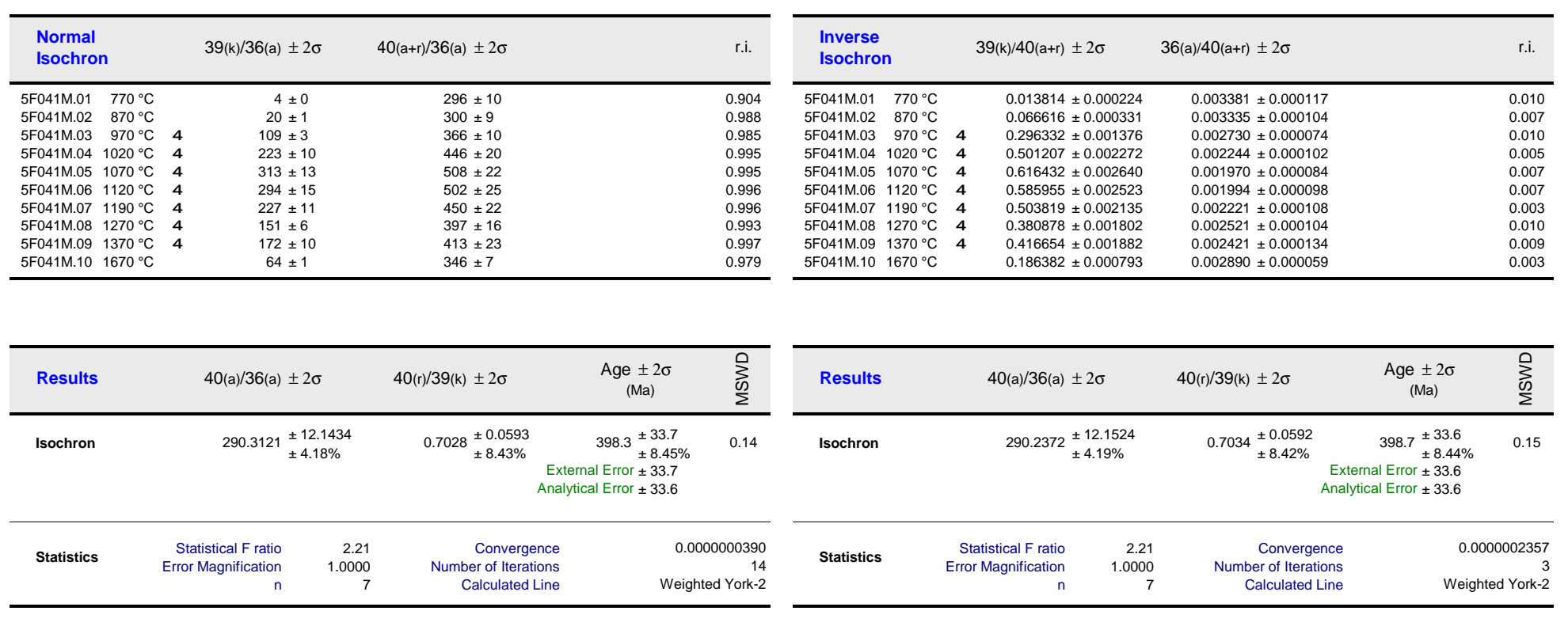




\begin{tabular}{|c|c|c|c|c|c|c|c|c|c|c|c|c|c|c|}
\hline \multicolumn{2}{|l|}{$\begin{array}{l}\text { Degassing } \\
\text { Patterns }\end{array}$} & \multirow{2}{*}{$\begin{array}{c}36 \operatorname{Ar}(\mathrm{a}) \\
0.00175\end{array}$} & \multirow{2}{*}{$\begin{array}{r}36 \operatorname{Ar}(\mathrm{ca}) \\
0.00001\end{array}$} & \multirow{2}{*}{$\begin{array}{c}36 \operatorname{Ar}(\mathrm{cl}) \\
0.00000 \\
0.00000\end{array}$} & \multirow{2}{*}{$\begin{array}{r}37 \mathrm{Ar}(\mathrm{ca}) \\
0.03069\end{array}$} & \multirow{2}{*}{$\begin{array}{c}38 \mathrm{Ar}(\mathrm{a}) \\
0.00033\end{array}$} & \multirow{2}{*}{$\begin{array}{r}38 \mathrm{Ar}(\mathrm{k}) \\
0.00009\end{array}$} & \multirow{2}{*}{$\begin{array}{r}38 \mathrm{Ar}(\mathrm{ca}) \\
0.00000\end{array}$} & \multirow{2}{*}{$\begin{array}{r}38 \operatorname{Ar}(\mathrm{cl}) \\
0.00009\end{array}$} & \multirow{2}{*}{$\begin{array}{r}39 \mathrm{Ar}(\mathrm{k}) \\
0.00716\end{array}$} & \multirow{2}{*}{$\begin{array}{r}39 \operatorname{Ar}(\mathrm{ca}) \\
0.00002\end{array}$} & \multirow{2}{*}{$\begin{array}{l}40 \mathrm{Ar}(\mathrm{r}) \\
0.00046\end{array}$} & \multirow{2}{*}{$\begin{array}{l}40 \operatorname{Ar}(a) \\
0.51819 \\
0.7728\end{array}$} & \multirow{2}{*}{$\begin{array}{r}40 \operatorname{Ar}(\mathrm{k}) \\
0.00000\end{array}$} \\
\hline 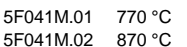 & & & & & & & & & & & & & & \\
\hline $3 \quad 970^{\circ} \mathrm{C}$ & 4 & $\begin{array}{l}0.00246 \\
0.00192\end{array}$ & $\begin{array}{l}0.00004 \\
0.00010\end{array}$ & $\begin{array}{l}0.00000 \\
0.00000\end{array}$ & $\begin{array}{l}0.14881 \\
0.37280\end{array}$ & $\begin{array}{l}0.00046 \\
0.00036\end{array}$ & $\begin{array}{l}0.00061 \\
0.00258\end{array}$ & $\begin{array}{l}0.000001 \\
0.0001\end{array}$ & $\begin{array}{l}0.000000 \\
0.00000\end{array}$ & $\begin{array}{l}0.04916 \\
0.20790\end{array}$ & $\begin{array}{l}0.00000 \\
0.0025\end{array}$ & $\begin{array}{l}0.010655 \\
0.13550\end{array}$ & $\begin{array}{l}.0 .56607 \\
0.5667\end{array}$ & $\begin{array}{l}0.00002 \\
0.00008\end{array}$ \\
\hline $5 F 041 \mathrm{M} .041020^{\circ} \mathrm{C}$ & 4 & 0.00132 & 0.00013 & 0.00000 & 0.45555 & 0.00025 & 0.00366 & 0.00002 & 0.00000 & 0.29483 & 0.00031 & 0.19824 & 0.39000 & 0.00011 \\
\hline $5 F 041 \mathrm{M} .051070^{\circ} \mathrm{C}$ & 4 & 0.00113 & 0.00017 & 0.00000 & 0.59615 & 0.00021 & 0.00440 & 0.00002 & 0.000 & 0.35395 & 40 & 23988 & 0.33431 & 0.00 \\
\hline $44 \mathrm{M} .06 \quad 1120^{\circ} \mathrm{C}$ & 4 & 0.00103 & 0.00013 & 0.00000 & 0.475 & 0.000 & 0.00378 & 0.00002 & & 0.303 & & & & 0.00 \\
\hline $71190^{\circ} \mathrm{C}$ & 4 & 0.00114 & & 0.000 & 0.303 & 0.00021 & 0.00321 & 0.00001 & & 0.25844 & & & .33660 & 0.00 \\
\hline M.08 $1270^{\circ} \mathrm{C}$ & 4 & 0.00135 & 0.00007 & 0.000 & 0.235 & 0.00025 & 0.00254 & 0.000 & & 0.204 & & & 39916 & 0.00008 \\
\hline \multirow{4}{*}{ 5F041M.10 $1670^{\circ}$} & 4 & & & & & & & & & & & & & 0.00007 \\
\hline & & 0.00565 & 0.00106 & 0.00000 & 3.79111 & 0.00106 & 0.00453 & 0.00013 & 0.00010 & 0.36442 & 0.00256 & 0.28539 & 1.66983 & 0.00014 \\
\hline & $\Sigma$ & 0.01876 & 0.00185 & 0.00000 & 6.64352 & 0.00351 & 0.02756 & 0.00023 & 0.00033 & 2.21696 & 0.00449 & 1.51450 & 5.54427 & 0.00086 \\
\hline & $\Sigma$ & & & & & & & & & & & & & \\
\hline
\end{tabular}

\begin{tabular}{|c|c|c|c|c|c|c|c|c|}
\hline \multicolumn{2}{|c|}{$\begin{array}{l}\text { Additional } \\
\text { Ratios }\end{array}$} & $40(r) / 39(k)$ & $1 \sigma$ & $40(r+a)$ & $1 \sigma$ & $37 \operatorname{Ar}($ decay $)$ & $39 \operatorname{Ar}$ (decay) & $40 \mathrm{Ar}$ (moles) \\
\hline $5 F 04 \mathrm{M} .01$ & $770^{\circ} \mathrm{C}$ & 0.06410 & 1.24863 & 0.51865 & 0.00061 & 4.160242 & 1.00050939 & $3.112 \mathrm{E}-14$ \\
\hline 5F041M.02 & $870^{\circ} \mathrm{C}$ & 0.21666 & 0.23088 & 0.73793 & 0.00040 & 4. 163553 & 1.00050967 & 4.428E-14 \\
\hline 5F041M.03 & & 0.65178 & 0.03674 & 0.70157 & 0.00039 & 4.165438 & 1.00050983 & 4.210E-14 \\
\hline 5 F041M.04 & $1020^{\circ} \mathrm{C}$ & 0.67238 & 0.03001 & 0.58824 & 0.00031 & 4.167381 & 1.00051000 & 3.530E-14 \\
\hline 5F041M.05 & $1070^{\circ} \mathrm{C}$ & 0.67773 & 0.02009 & 0.57419 & 0.00033 & 4.169325 & 1.00051017 & 3.446E-14 \\
\hline 5F041M.06 & $1120^{\circ} \mathrm{C}$ & 0.70110 & 0.02487 & 0.51855 & 0.00031 & 4.171212 & 1.00051033 & $3.112 \mathrm{E}-14$ \\
\hline 5F041M.07 & $\begin{array}{ll}1190^{\circ} \mathrm{C} \\
1270\end{array}$ & 0.68240 & 0.03184 & 0.51296 & 0.00020 & 4.173158 & 1.00051049 & $3.078 \mathrm{E}-14$ \\
\hline $\begin{array}{l}\text { 5F-044M.M. } \\
\text { 5F041M.09 }\end{array}$ & $\begin{array}{l}1277^{\circ} \mathrm{C} \\
1370^{\circ} \mathrm{C}\end{array}$ & $\begin{array}{l}0.669993 \\
0.68294\end{array}$ & $\begin{array}{l}0.040222 \\
0.04768\end{array}$ & $\begin{array}{l}0.53590 \\
0.41557\end{array}$ & $\begin{array}{l}0.000037 \\
0.00031\end{array}$ & $\begin{array}{l}4.175048 \\
4.176995\end{array}$ & $\begin{array}{l}1.000051066 \\
1.00051082\end{array}$ & $\begin{array}{l}3.216 \mathrm{E}-14 \\
2.494 \mathrm{E}-14\end{array}$ \\
\hline 5F041M.10 & $1670^{\circ} \mathrm{C}$ & 0.78315 & 0.04672 & 1.95522 & 0.00051 & 4.178886 & 1.00051098 & 1. $173 \mathrm{E}-13$ \\
\hline
\end{tabular}


Age Plateau 


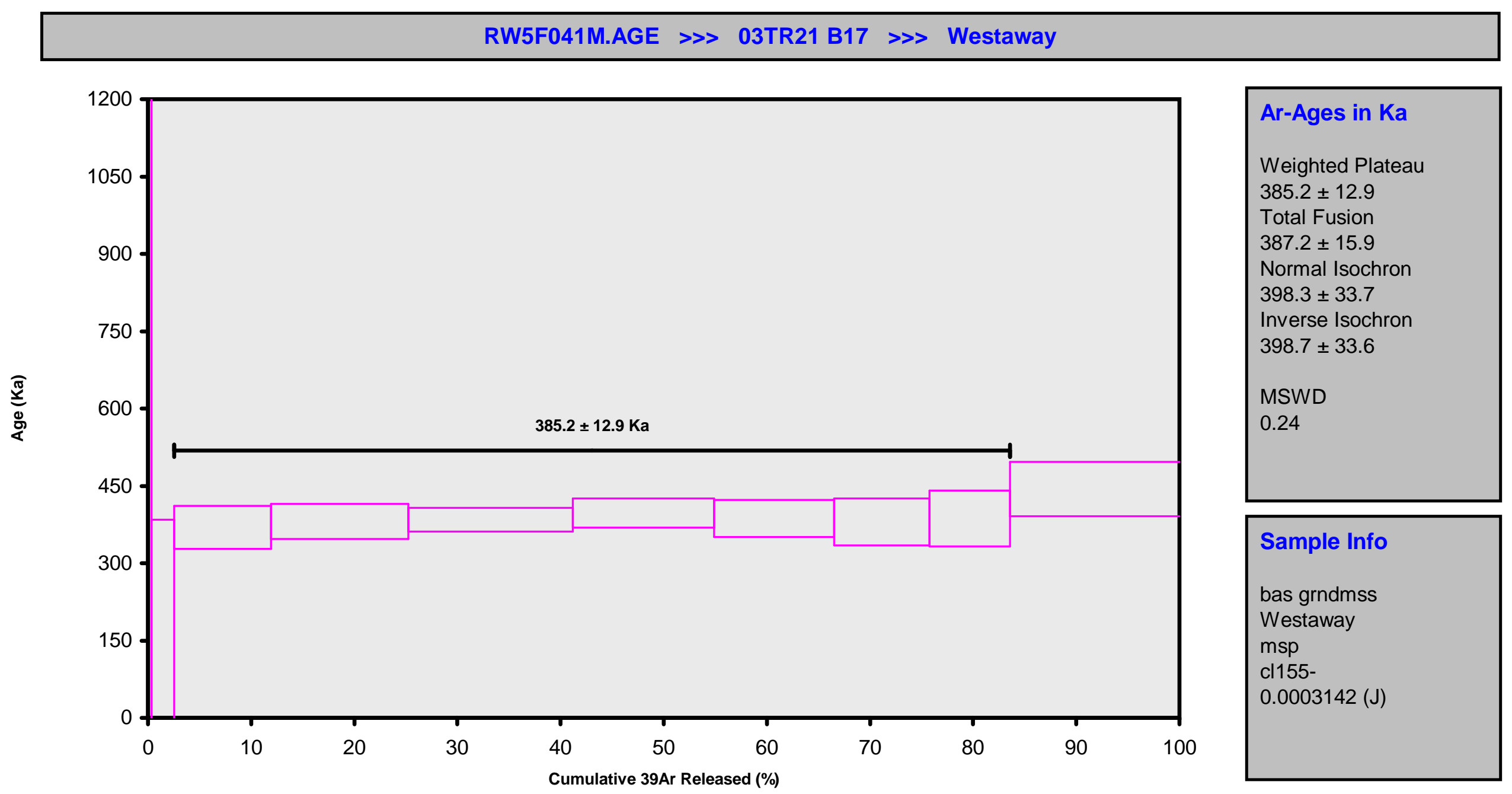


K-Ca Plateau 


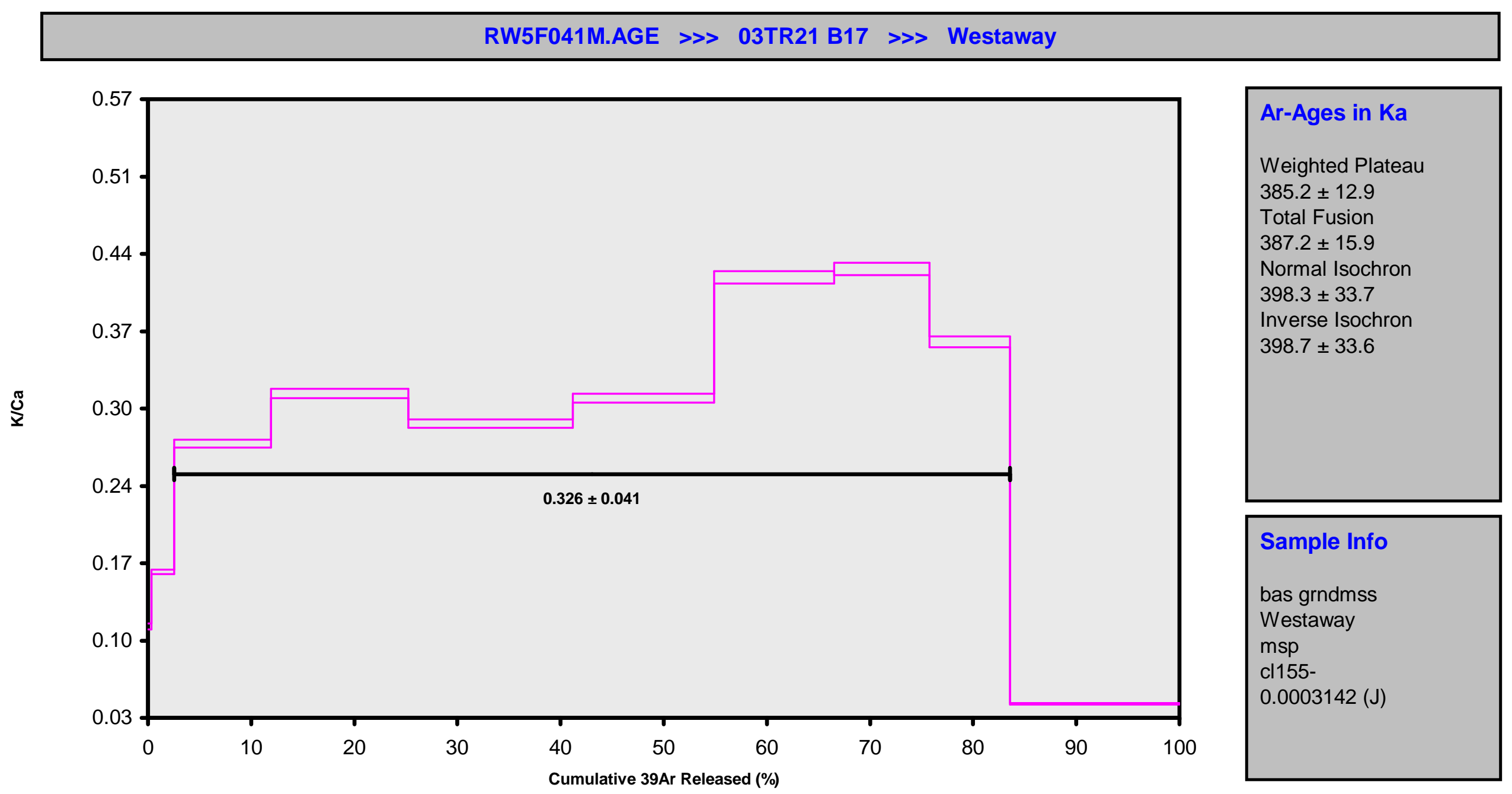


Normal Isochron 


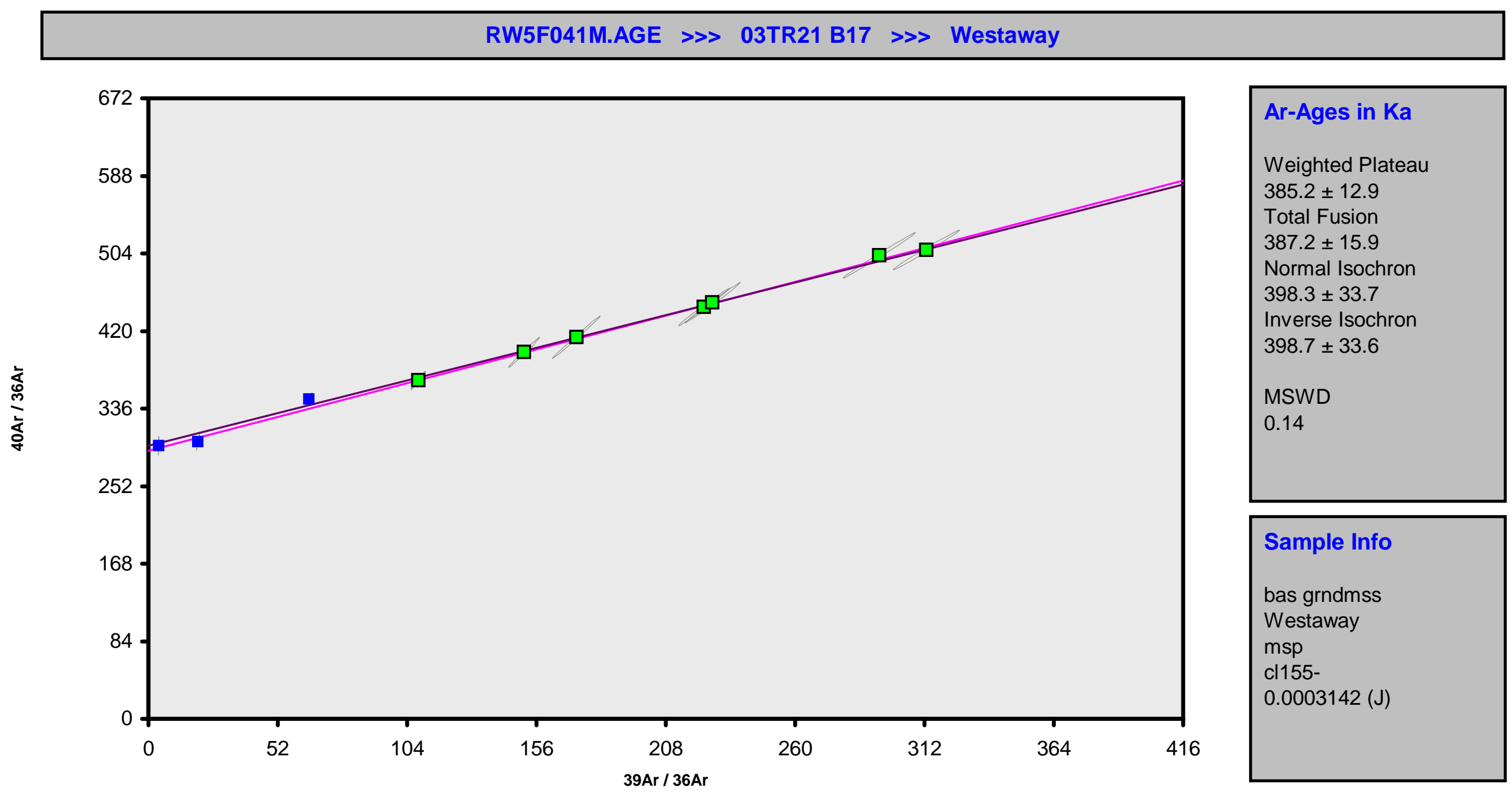




\section{Inverse Isochron}


MIT, Cambridge, USA

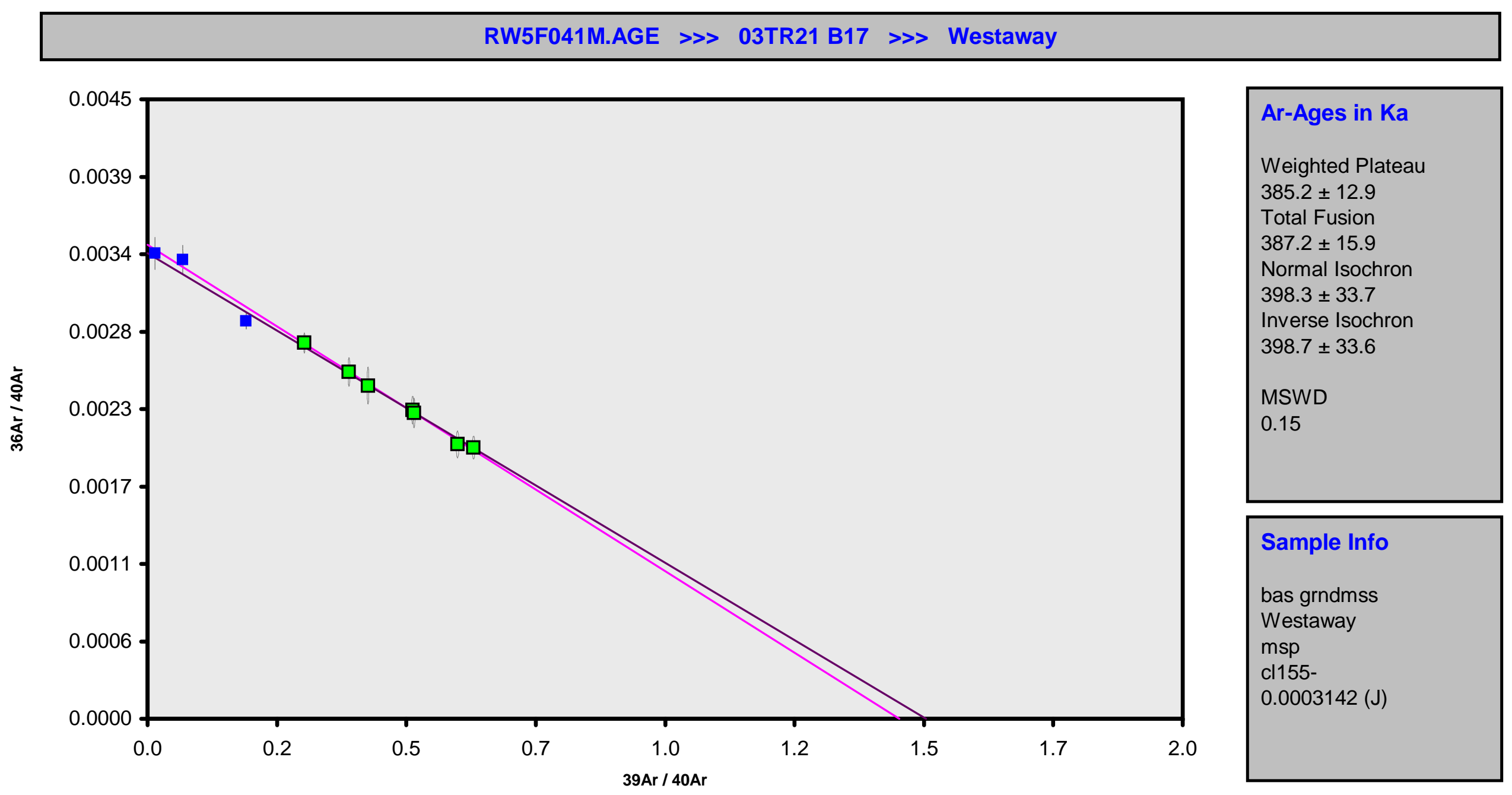


Sample 01TR51 
Data Tables 


\begin{tabular}{|c|c|c|c|c|c|c|c|c|c|c|c|}
\hline $\begin{array}{l}\text { Procedu } \\
\text { Blanks }\end{array}$ & & $36 \mathrm{Ar}$ & $1 \sigma$ & $37 \mathrm{Ar}$ & $1 \sigma$ & $38 \mathrm{Ar}$ & $1 \sigma$ & $39 \mathrm{Ar}$ & $1 \sigma$ & $40 \mathrm{Ar}$ & $1 \sigma$ \\
\hline $\begin{array}{l}5 F 0410.01 \\
5 F 0410.02\end{array}$ & $\begin{array}{l}770^{\circ} \mathrm{C} \\
870^{\circ} \mathrm{C}\end{array}$ & $\begin{array}{l}0.000239 \\
0.000239\end{array}$ & $\begin{array}{l}0.000017 \\
0.000017\end{array}$ & $\begin{array}{l}0.000234 \\
0.000235\end{array}$ & $\begin{array}{l}0.000029 \\
0.000029\end{array}$ & $\begin{array}{l}0.000135 \\
0.000135\end{array}$ & $\begin{array}{l}0.000023 \\
0.000023\end{array}$ & $\begin{array}{l}0.000415 \\
0.000416\end{array}$ & $\begin{array}{l}0.000045 \\
0.000045\end{array}$ & $\begin{array}{l}0.040000 \\
0.040000\end{array}$ & $\begin{array}{l}0.000125 \\
0.000125\end{array}$ \\
\hline $5 F 0410.03$ & & 0.000240 & 0.000017 & 0.000236 & 0.000029 & 0.000136 & 0.000023 & 0.000417 & 0.000045 & 0.040000 & 0.000125 \\
\hline $5 F 0410.04$ & $1020^{\circ} \mathrm{C}$ & 0.000240 & 0.000017 & 0.000236 & 0.000029 & 0.000136 & 0.000023 & 0.000418 & 0.000045 & & 0.000125 \\
\hline $5 F 0410.05$ & $1070^{\circ} \mathrm{C}$ & 0.000240 & 0.000017 & 0.000237 & 0.000029 & 0.000136 & 0.000023 & & & & 0.000125 \\
\hline $5 F 0410.06$ & $1120^{\circ} \mathrm{C}$ & 0.000240 & 0.000017 & 0.000238 & 0.000029 & & & & 0.000045 & & 0.000125 \\
\hline $5 F 0410.07$ & $1190^{\circ} \mathrm{C}$ & 0.000241 & 0.000017 & 0.000238 & 0.000029 & 0.000137 & 0.000023 & 0.000420 & 0.000045 & 0.040000 & 0.000125 \\
\hline $\begin{array}{l}5 F 0410.08 \\
5 F 0410.09\end{array}$ & $\begin{array}{l}1270^{\circ} \mathrm{C} \\
1370{ }^{\circ} \mathrm{C}\end{array}$ & $\begin{array}{l}0.000241 \\
0.000241\end{array}$ & $\begin{array}{l}0.000017 \\
0.000017\end{array}$ & $\begin{array}{l}0.000239 \\
0.000240\end{array}$ & $\begin{array}{l}0.00002929 \\
0.000029\end{array}$ & $\begin{array}{l}0.000137 \\
0.000137\end{array}$ & $\begin{array}{l}0.0000023 \\
0.000023\end{array}$ & $\begin{array}{l}0.000421 \\
0.000421\end{array}$ & $\begin{array}{l}0.0000045 \\
0.000045\end{array}$ & $\begin{array}{l}0.0400000 \\
0.040000\end{array}$ & $\begin{array}{l}0.000125 \\
0.000125\end{array}$ \\
\hline 5F0410.10 & $1670^{\circ} \mathrm{C}$ & 0.000241 & 0.000017 & 0.000240 & 0.000029 & 0.000138 & 0.000023 & 0.000422 & 0.000045 & 0.040000 & 0.000125 \\
\hline
\end{tabular}


Clair
MIT. Cambridge, USA

\begin{tabular}{|c|c|c|c|c|c|c|c|c|c|c|c|c|c|c|c|c|c|c|c|c|c|}
\hline $\begin{array}{l}\text { Intercept } \\
\text { Values }\end{array}$ & & $36 \mathrm{Ar}$ & $1 \sigma$ & r2 & & $37 \mathrm{Ar}$ & $1 \sigma$ & r2 & & $38 \mathrm{Ar}$ & $1 \sigma$ & $\mathrm{r} 2$ & & $39 \mathrm{Ar}$ & $1 \sigma$ & $\mathrm{r}^{2}$ & & $40 \mathrm{Ar}$ & $1 \sigma$ & r2 & \\
\hline $5 F 0410.01$ & $770^{\circ} \mathrm{C}$ & 0.002926 & 0.000015 & 0.7972 & LIN \# 1215 & 0.011325 & 0.000049 & 0.8586 & LIN \# 12 & 0.001155 & 0.000027 & 0.0301 & LIN \# 12 & 0.026643 & 0.000033 & 0.9947 & EXP \#181315 & 0.818897 & 0.000574 & 0.9834 & LIN \#1567 \\
\hline $5 F 0410.02$ & $870^{\circ} \mathrm{C}$ & 0.002916 & 0.000032 & 0.6048 & LIN \# 12315 & 0.038391 & 0.000087 & 0.9872 & LIN \# 1 & 0.002047 & 0.000037 & 0.4663 & LIN \# 1215 & 0.089410 & 0.000140 & 0.9838 & EXP\#123 & 0.846721 & 0.000365 & 0.9957 & $\operatorname{EXP} \# 1611$ \\
\hline $5 F 0410.03$ & $970^{\circ} \mathrm{C}$ & 0.002678 & 0.000012 & 0.8979 & LIN \# 12 & 0.084036 & 0.000075 & 0.9982 & LIN \# & 0.004054 & 0.000037 & 0.8416 & LIN \# 12 & 0.270543 & 0.000212 & 0.9985 & EXP \# 1 & 0.908927 & 0.000365 & 0.9928 & EXP \# 14 \\
\hline $5 F 0410.04 \quad 1$ & $1020^{\circ} \mathrm{C}$ & 0.001863 & 0.000017 & 0.4744 & LIN \# 115 & 0.097004 & 0.000085 & 0.9985 & LIN \# 113 & 0.004579 & 0.000037 & 0.7907 & LIN \# 13 & 0.344398 & 0.000210 & 0.9992 & LIN \# 5 & 0.731224 & 0.000356 & 0.9974 & LIN \# 1 \\
\hline $5 F 0410.05 \quad 1$ & $1070^{\circ} \mathrm{C}$ & 0.001684 & 0.000029 & 0.1937 & LIN \# 15 & 0.113169 & 0.000165 & 0.9972 & EXP \# 15813 & 0.005875 & 0.000033 & 0.9378 & LIN \# 15 & 0.451818 & 0.000302 & 0.9992 & EXP \# 713 & 0.747589 & 0.000263 & 0.9981 & EXP \# 181213 \\
\hline $5 F 0410.06 \quad 1$ & $1120^{\circ} \mathrm{C}$ & 0.001373 & 0.000036 & 0.0154 & LIN \# 115 & 0.107717 & 0.000115 & 0.9978 & EXP \# 1 & 0.006396 & 0.000028 & 0.9552 & LIN \#1 15 & 0.494018 & 0.000188 & 0.9997 & EXP \# 1 & 0.698369 & 0.000247 & 0.9988 & EXP \# 167 \\
\hline $\begin{array}{l}5 F 0410.07 \quad 1 \\
5 F 5410.8\end{array}$ & $1190^{\circ} \mathrm{C}$ & 0.001332 & 0.000017 & 0.0565 & LIN \#1 15 & 0.084466 & 0.000108 & 0.9969 & $\begin{array}{l}\text { LIN \# } 115 \\
\text { LXP \#12 }\end{array}$ & 0.006488 & 0.000035 & 0.9429 & LIN \# 1 & 0.494367 & 0.000298 & 0.9992 & $\begin{array}{l}\text { LIN \# } \\
\text { LYP }\end{array}$ & 0.691643 & 0.000282 & 0.9977 & LIN \# 1131415 \\
\hline $\begin{array}{l}\begin{array}{l}50410.08 \\
5 F 0410.09\end{array} \\
\end{array}$ & $1370^{\circ} \mathrm{C}$ & $\begin{array}{l}0.001434 \\
0.001410\end{array}$ & $\begin{array}{l}.0 .000017 \\
0.000017\end{array}$ & $\begin{array}{l}0.2053 \\
0.0306\end{array}$ & $\begin{array}{l}\operatorname{LN} \# 1 \\
\operatorname{LN} \# 1\end{array}$ & $\begin{array}{l}0.053495 \\
0.063511\end{array}$ & $\begin{array}{l}0.000065 \\
0.000100\end{array}$ & $\begin{array}{l}0.9974 \\
0.9951\end{array}$ & $\begin{array}{l}\text { EPP \#12 } \\
\operatorname{LIN} \# 1\end{array}$ & $\begin{array}{l}0.005156 \\
0.003728\end{array}$ & $\begin{array}{l}0.000029 \\
0.000039\end{array}$ & $\begin{array}{l}0.9272 \\
0.7798\end{array}$ & $\begin{array}{l}\operatorname{LN} \# 1 \\
\operatorname{LIN} \# 15\end{array}$ & $\begin{array}{l}0.379445 \\
0.259708\end{array}$ & $\begin{array}{l}0.000179 \\
0.000102\end{array}$ & $\begin{array}{l}0.9996 \\
0.9997\end{array}$ & $\begin{array}{l}\text { EXP \#1 } \\
\text { LIN \#1 }\end{array}$ & 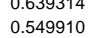 & $\begin{array}{l}0.000447 \\
0.000298\end{array}$ & $\begin{array}{l}0.9951 \\
0.9981\end{array}$ & LIN \#1919 \\
\hline 5F0410.10 1 & $1670^{\circ} \mathrm{C}$ & 0.004251 & 0.000023 & 0.8511 & LIN \# 1 & 0.686409 & 0.000288 & 0.9997 & EXP \# 3 & 0.002171 & 0.000028 & 0.1387 & LIN \# 1 & 0.095884 & 0.000068 & 0.9966 & EXP \# 1414 & 1.052886 & 0.000278 & 0.9394 & EXP \# 18 \\
\hline
\end{tabular}




\begin{tabular}{|c|c|c|c|c|c|c|c|c|c|c|c|c|c|c|c|c|c|c|c|c|c|c|c|}
\hline \multicolumn{2}{|c|}{$\begin{array}{l}\text { Sample } \\
\text { Parameters }\end{array}$} & Sample & Material & Location & Analyst & 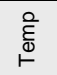 & $\begin{array}{l}\text { Standard } \\
\text { (in Ma) }\end{array}$ & $\% 1 \sigma$ & $J$ & $\% 1 \sigma$ & Fract & $\% 1 \sigma$ & $\begin{array}{l}\text { Volume } \\
\text { Corr. }\end{array}$ & $\begin{array}{l}\text { Sensitivity } \\
\text { (molvol) }\end{array}$ & ฮิ & 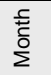 & 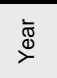 & 호 & $\frac{\check{5}}{\Sigma}$ & 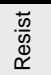 & Irradiation & Project & $\begin{array}{l}\text { Standard } \\
\text { Name }\end{array}$ \\
\hline $5 F 0410.01$ & $770{ }^{\circ} \mathrm{C}$ & 01TR51 B19 & bas grndmss & Iestaway Volcanic & msp & 770 & 28.34 & 0.01 & 0.0003066 & 0.3 & 1.007 & 0.2 & 1 & $6.000 \mathrm{E}-14$ & 29 & 07 & 2005 & 21 & 32 & 001 & & & \\
\hline & & R51 B19 & s grndmss & Iestaway Volcar & & & & & & 0.3 & & 0.2 & 1 & & 29 & 07 & & 22 & 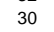 & & & & \\
\hline $5 F 0410.03$ & $970^{\circ} \mathrm{C}$ & 01TR51 B19 & bas grndmss & Iestaway Volcanic & msp & 70 & 28.34 & 0.01 & 0.0003066 & 0.3 & 1.007 & 0.2 & 1 & $6.000 \mathrm{E}-14$ & 29 & 07 & 2005 & 23 & & & & Vestaway & tcr-2a \\
\hline $5 F 0410.04$ & $1020^{\circ} \mathrm{C}$ & 01TR51 B19 & bas grndmss & Iestaway Volcanic & msp & 1020 & 28.34 & 0.01 & 0.0003066 & 0.3 & 1.007 & 0.2 & 1 & $6.000 \mathrm{E}-14$ & 29 & 07 & 2005 & 23 & 37 & 1 & & Westaway & tcr-2a \\
\hline 5F0410.05 & $1070^{\circ} \mathrm{C}$ & 01TR51 B19 & bas grndmss & Iestaway Volcanic & msp & 1070 & 28.34 & 0.01 & 0.0003066 & 0.3 & & 0.2 & 1 & $6.000 \mathrm{E}-14$ & 30 & 07 & 2005 & 00 & 10 & 001 & cl155- & Westaway & tcr-2a \\
\hline 5F0410.06 & $1120^{\circ} \mathrm{C}$ & 01 TR51 B & bas grndmss & lestaway Volcanic & & 1120 & 28.34 & 0.01 & 0.0003066 & 0.3 & 1.00 & 0.2 & 1 & & 30 & 07 & 2005 & 00 & 44 & 001 & & & tcr-2a \\
\hline $5 F 0410.0$ & $1190^{\circ} \mathrm{C}$ & 01TR51 & bas grndms & Iestaway Volcanic & $\mathrm{ms}$ & 11 & 28 & 0.01 & 0.00030 & 0.3 & 1.0 & 0.2 & 1 & 6.000 & 30 & 07 & 2005 & 01 & 18 & 001 & cl155- & & tcr-2a \\
\hline & $1270^{\circ} \mathrm{C}$ & 01TR5 & g grndmss & Iestaway Vol & $\mathrm{ms}$ & & & 0.01 & & 0.3 & & 0.2 & 1 & & 30 & 07 & 2005 & 01 & 51 & 001 & & & tcr-2a \\
\hline & $1370^{\circ} \mathrm{C}$ & & g grndmss & lestaway Volcanic & msp & 1370 & 28.34 & 0.01 & 0.0003066 & 0.3 & 1.007 & 0.2 & 1 & $6.000 E-14$ & 30 & 07 & 2005 & 02 & 25 & 001 & cl155- & Westaway & tcr-2a \\
\hline 5F0410.10 & $1670^{\circ} \mathrm{C}$ & 01TR51 B19 & bas grndmss & lestaway Volcanii & msp & 1670 & 28.34 & 0.01 & 0.0003066 & 0.3 & 1.007 & 0.2 & 1 & $6.000 \mathrm{E}-14$ & 30 & & & & 58 & 001 & cl155- & Westaway & tcr-2a \\
\hline
\end{tabular}


MIT, Cambridge, USA

\begin{tabular}{|c|c|c|c|c|c|c|c|c|c|c|c|c|c|c|c|c|c|c|c|c|c|c|c|}
\hline $\begin{array}{l}\text { Irradiati } \\
\text { Constar }\end{array}$ & & $40 / 36$ (a) & $\% 1 \sigma$ & 38/36(a) & $\% 1 \sigma$ & 39/37(ca) & $\% 1 \sigma$ & 38/37(ca) & $\% 1 \sigma$ & $36 / 37$ (ca) & $\% 1 \sigma$ & $40 / 39(k)$ & $\% 1 \sigma$ & $38 / 39(k)$ & $\% 1 \sigma$ & $36 / 38$ (cl) & $\% 1 \sigma$ & $\mathrm{K} / \mathrm{Ca}$ & $\% 1 \sigma$ & $\mathrm{K} / \mathrm{Cl}$ & $\% 1 \sigma$ & $\mathrm{Ca} / \mathrm{Cl}$ & $\% 1 \sigma$ \\
\hline $5 F 0410.01$ & $770^{\circ} \mathrm{C}$ & 295.5 & 0 & 0.1869 & 0 & 0.000676 & 0 & 0.000034 & 0 & 0.000279 & 0 & 0.00039 & 0 & 0.01243 & 0 & 0 & 0 & 0.49 & 0 & 0 & 0 & 0 & 0 \\
\hline $5 F 0410.02$ & $870^{\circ} \mathrm{C}$ & 295.5 & 0 & 0.1869 & 0 & 0.000676 & 0 & 0.000034 & 0 & 0.000279 & 0 & 0.00039 & 0 & 0.01243 & 0 & 0 & 0 & 0.49 & 0 & 0 & 0 & 0 & 0 \\
\hline $5 F 0410.03$ & $970^{\circ} \mathrm{C}$ & 295.5 & 0 & 0.1869 & 0 & 0.000676 & 0 & 0.000034 & 0 & 0.000279 & 0 & 0.00039 & 0 & 0.01243 & 0 & 0 & 0 & 0.49 & 0 & 0 & 0 & 0 & 0 \\
\hline $5 F 0410.04$ & $1020^{\circ} \mathrm{C}$ & 295.5 & 0 & 0.1869 & 0 & 0.000676 & 0 & 0.000034 & 0 & 0.000279 & 0 & 0.00039 & 0 & 0.01243 & 0 & 0 & 0 & 0.49 & 0 & 0 & 0 & 0 & 0 \\
\hline $\begin{array}{l}5 F 0410.05 \\
5 F 0410\end{array}$ & $\begin{array}{l}1070^{\circ} \mathrm{C} \\
110\end{array}$ & $\begin{array}{l}295.5 \\
295.5\end{array}$ & 0 & 0.1869 & ${ }^{0}$ & $\begin{array}{l}0.000676 \\
\end{array}$ & 0 & 0.000034 & 0 & 0.000279 & 0 & 0.00039 & 0 & 0.01243 & 0 & 0 & 0 & 0.49 & 0 & 0 & 0 & 0 & 0 \\
\hline $\begin{array}{l}5 F 0410.06 \\
5 F 410.07\end{array}$ & $\begin{array}{ll}1120^{\circ} \mathrm{C} \\
11100\end{array}$ & $\begin{array}{l}295.5 \\
2955\end{array}$ & 0 & $\begin{array}{l}0.1869 \\
0\end{array}$ & ${ }^{0}$ & $\begin{array}{l}0.000676 \\
\end{array}$ & 0 & $\begin{array}{l}0.000034 \\
\end{array}$ & 0 & $\begin{array}{l}0.000279 \\
0\end{array}$ & 0 & 0.00039 & 0 & 0.01243 & 0 & 0 & 0 & 0.49 & 0 & 0 & 0 & 0 & 0 \\
\hline $\begin{array}{l}5 F 0410.07 \\
5 F 0410.08\end{array}$ & $\begin{array}{l}1190^{\circ} \mathrm{C} \\
127{ }^{\circ} \mathrm{C}\end{array}$ & $\begin{array}{l}295.5 \\
295.5\end{array}$ & $\begin{array}{l}0 \\
0\end{array}$ & $\begin{array}{l}0.1869 \\
0.1869\end{array}$ & $\begin{array}{l}0 \\
0\end{array}$ & $\begin{array}{l}0.000676 \\
0.000676\end{array}$ & $\begin{array}{l}0 \\
0\end{array}$ & $\begin{array}{l}0.0000034 \\
0.000034\end{array}$ & $\begin{array}{l}0 \\
0\end{array}$ & $\begin{array}{l}0.000279 \\
0.000279\end{array}$ & $\begin{array}{l}0 \\
0\end{array}$ & $\begin{array}{l}0.000039 \\
0.00039\end{array}$ & $\begin{array}{l}0 \\
0\end{array}$ & $\begin{array}{l}0.01243 \\
0.01243\end{array}$ & $\begin{array}{l}0 \\
0\end{array}$ & $\begin{array}{l}0 \\
0\end{array}$ & $\begin{array}{l}0 \\
0\end{array}$ & $\begin{array}{l}0.49 \\
0.49\end{array}$ & $\begin{array}{l}0 \\
0\end{array}$ & $\begin{array}{l}0 \\
0\end{array}$ & $\begin{array}{l}0 \\
0\end{array}$ & $\begin{array}{l}0 \\
0\end{array}$ & $\begin{array}{l}0 \\
0\end{array}$ \\
\hline 5F0410.09 & $1370^{\circ} \mathrm{C}$ & 295.5 & 0 & 0.1869 & 0 & 0.000676 & 0 & 0.000034 & 0 & 0.000279 & 0 & 0.00039 & 0 & 0.01243 & 0 & 0 & 0 & 0.49 & 0 & 0 & 0 & 0 & 0 \\
\hline $5 F 0410.10$ & $1670^{\circ} \mathrm{C}$ & 295.5 & 0 & 0.1869 & 0 & 0.000676 & 0 & 0.000034 & 0 & 0.000279 & 0 & 0.00039 & 0 & 0.01243 & 0 & 0 & 0 & 0.49 & 0 & 0 & 0 & 0 & 0 \\
\hline
\end{tabular}




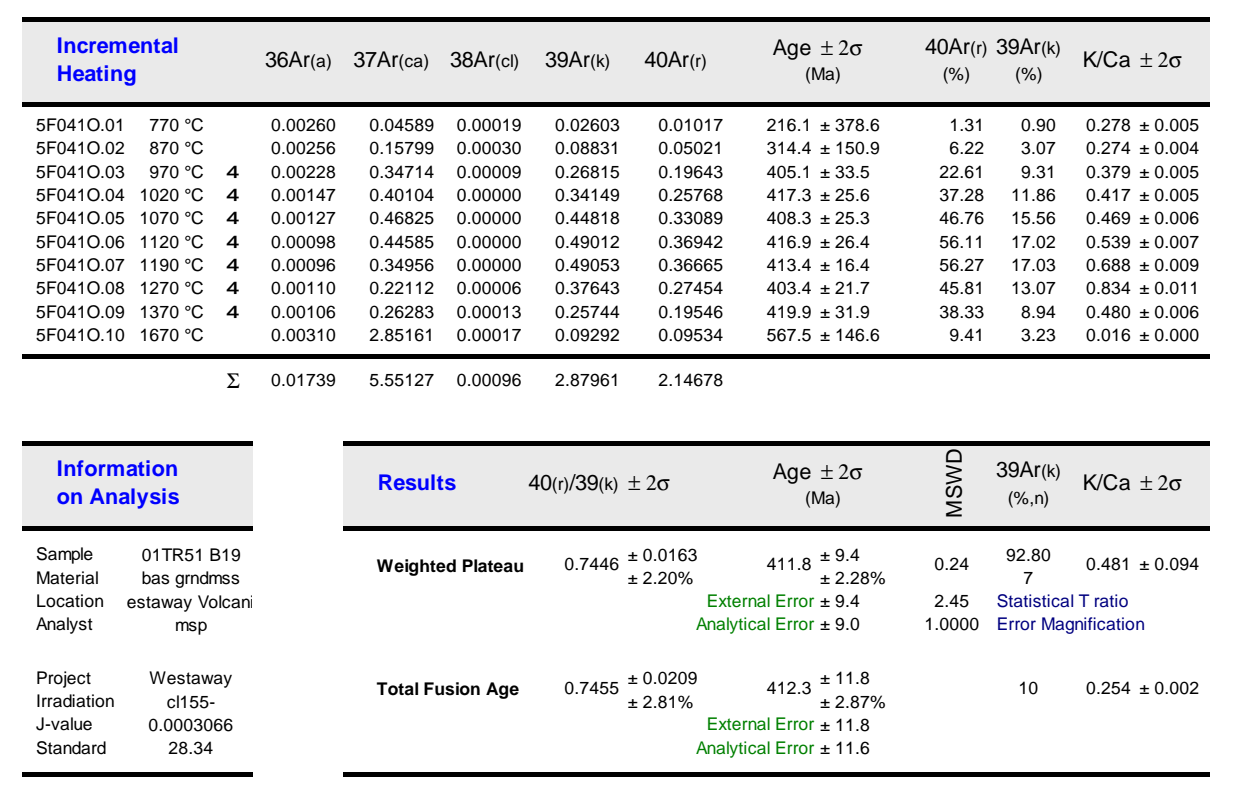




\begin{tabular}{|c|c|c|c|c|}
\hline \multicolumn{2}{|c|}{$\begin{array}{l}\text { Normal } \\
\text { Isochron }\end{array}$} & \multirow{2}{*}{$\frac{39(\mathrm{k}) / 36(\mathrm{a}) \pm 2 \sigma}{10 \pm 0}$} & \multirow{2}{*}{$\frac{40(a+r) / 36(a) \pm 2 \sigma}{299 \pm 7}$} & \multirow{3}{*}{$\begin{array}{c}\text { r.i. } \\
0.967\end{array}$} \\
\hline $5 F 0410.01$ & $770^{\circ} \mathrm{C}$ & & & \\
\hline $5 F 0410.02$ & $870^{\circ} \mathrm{C}$ & & $315 \pm 10$ & \\
\hline $5 F 0410.03$ & $970^{\circ} \mathrm{C} \quad 4$ & $118 \pm 3$ & $382 \pm 9$ & 0.984 \\
\hline $5 F 0410.04$ & $1020^{\circ} \mathrm{C} \quad 4$ & $233 \pm 9$ & $471 \pm 17$ & 0.993 \\
\hline $\begin{array}{l}5 F 0410.05 \\
5 F 010.06\end{array}$ & $\begin{array}{ll}1070{ }^{\circ} \mathrm{C} & 4 \\
1120{ }^{\circ} \mathrm{C} & 4\end{array}$ & $\begin{array}{l}352 \pm 19 \\
502+44\end{array}$ & $555 \pm 30$ & 0.997 \\
\hline $\begin{array}{l}\begin{array}{l}5 F 0410.06 \\
5 F 410.07\end{array}\end{array}$ & 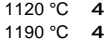 & $\begin{array}{l}502 \pm 41 \\
509 \pm 26\end{array}$ & $\begin{array}{l}674 \pm 54 \\
676+ \pm 34\end{array}$ & 0.999 \\
\hline $\begin{array}{l}5 F 0410.07 \\
5 F 0410.08\end{array}$ & 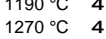 & $\begin{array}{l}509 \pm 26 \\
343 \pm 16\end{array}$ & $\begin{array}{l}666 \pm 34 \\
545 \pm 25\end{array}$ & \\
\hline $5 F 0410.09$ & $1370^{\circ} \mathrm{C} \quad 4$ & $242 \pm 11$ & $479 \pm 23$ & 0.996 \\
\hline $5 F 0410.10$ & $1670^{\circ} \mathrm{C}$ & $30 \pm 1$ & $326 \pm 9$ & 0.986 \\
\hline
\end{tabular}

\begin{tabular}{|c|c|c|c|c|}
\hline $\begin{array}{l}\text { Inverse } \\
\text { Isochro }\end{array}$ & & $39(\mathrm{k}) / 40(\mathrm{a}+\mathrm{r}) \pm 2 \sigma$ & $36(\mathrm{a}) / 40(\mathrm{a}+\mathrm{r}) \pm 2 \sigma$ & r.i. \\
\hline $5 F 0410.01$ & $770^{\circ} \mathrm{C}$ & $0.033417 \pm 0.000202$ & $0.003340 \pm 0.000077$ & 0.016 \\
\hline $5 F 0410.02$ & $870^{\circ} \mathrm{C}$ & $0.109477 \pm 0.000579$ & $0.003173 \pm 0.000101$ & 0.005 \\
\hline $5 F 0410.03$ & $970^{\circ} \mathrm{C} \quad 4$ & $0.308638 \pm 0.001360$ & $0.002619 \pm 0.000063$ & 0.007 \\
\hline $5 F 0410.04$ & $1020^{\circ} \mathrm{C} 4$ & $0.494138 \pm 0.002142$ & $0.002122 \pm 0.000077$ & 0.008 \\
\hline $5 F 0410.05$ & $1070^{\circ} \mathrm{C} 4$ & $0.633541 \pm 0.002727$ & $0.001801 \pm 0.000098$ & 0.003 \\
\hline $5 F 0410.06$ & $1120^{\circ} \mathrm{C} \quad 4$ & $0.744661 \pm 0.003101$ & $0.001485 \pm 0.000120$ & 0.002 \\
\hline $5 F 0410.07$ & $1190^{\circ} \mathrm{C} \quad 4$ & $0.752979 \pm 0.003231$ & $0.001479 \pm 0.000075$ & 0.004 \\
\hline $\begin{array}{l}5 F 0440.08 \\
5 F 0410.09\end{array}$ & $\begin{array}{l}1270^{\circ}{ }^{\circ} \mathrm{C} \\
1370^{\circ} \mathrm{C}\end{array}$ & $\begin{array}{l}0.628262 \pm 0.0022655 \\
0.504972 \pm 0.002164\end{array}$ & $\begin{array}{l}0.001833 \pm 0.0000083 \\
0.002087 \pm 0.000098\end{array}$ & $\begin{array}{l}0.012 \\
0.008\end{array}$ \\
\hline $5 F 0410.10$ & $1670^{\circ} \mathrm{C}$ & $0.091741 \pm 0.000412$ & $0.003066 \pm 0.000082$ & 0.003 \\
\hline
\end{tabular}

\begin{tabular}{|c|c|c|c|c|}
\hline Results & $40(\mathrm{a}) / 36(\mathrm{a}) \pm 2 \sigma$ & $40(r) / 39(k) \pm 2 \sigma$ & $\underset{\text { (Ma) }}{\operatorname{Age} \pm 2 \sigma}$ & $\sum_{\substack{0 \\
\text { in }}}^{0}$ \\
\hline Isochron & $\begin{aligned} 294.3990 & \pm 9.5350 \\
& \pm 3.24 \%\end{aligned}$ & $\begin{aligned} 0.7478 & \pm 0.0345 \\
& \pm 4.61 \%\end{aligned}$ & $\begin{array}{c}413.6 \pm 19.2 \\
\pm 4.65 \% \\
\text { nal Error } \pm 19.2 \\
\text { cal Error } \pm 19.1\end{array}$ & 0.28 \\
\hline
\end{tabular}

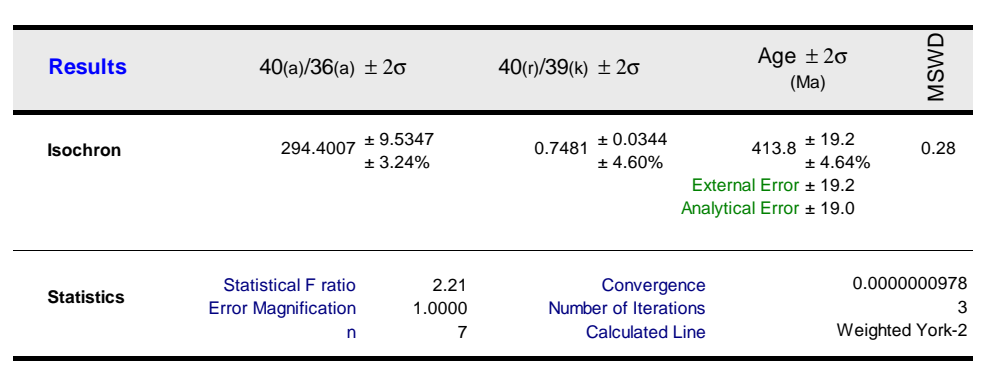


MIT, Cambridge, USA

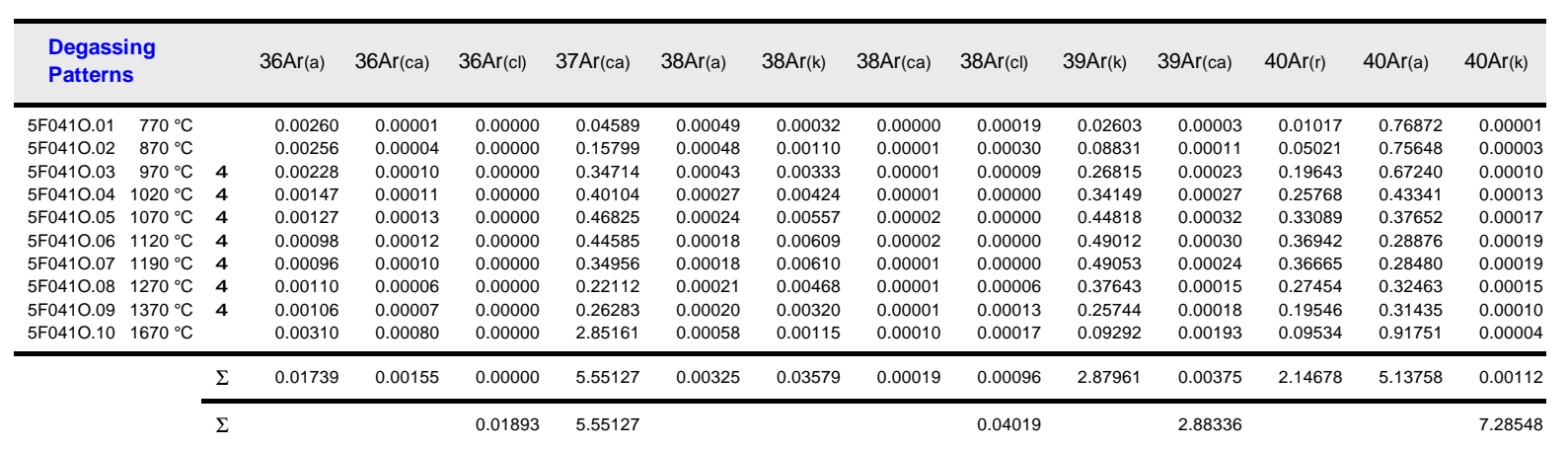

\begin{tabular}{|c|c|c|c|c|c|c|c|c|}
\hline \multicolumn{2}{|c|}{$\begin{array}{l}\text { Additional } \\
\text { Ratios }\end{array}$} & \multirow{2}{*}{$\begin{array}{r}40(\mathrm{r}) / 39(\mathrm{k}) \\
0.39071\end{array}$} & \multirow{2}{*}{$\frac{1 \sigma}{0.34225}$} & \multirow{2}{*}{$\begin{array}{l}40(r+a) \\
0.77889\end{array}$} & \multirow{3}{*}{$\begin{array}{c}1 \sigma \\
0.00059 \\
0.00039\end{array}$} & \multirow{3}{*}{$\begin{array}{r}37 \text { Ar(decay) } \\
4.224184 \\
4.227546\end{array}$} & \multirow{3}{*}{$\begin{array}{c}39 \operatorname{Ar} \text { (decay) } \\
1.00051484 \\
1.00051512\end{array}$} & \multirow{2}{*}{$\begin{array}{l}40 \mathrm{Ar} \text { (moles) } \\
4.673 \mathrm{E}-14\end{array}$} \\
\hline $5 F 0410.01$ & $770^{\circ} \mathrm{C}$ & & & & & & & \\
\hline $5 F 0410.02$ & $870^{\circ} \mathrm{C}$ & 0.56849 & & 0.80669 & & & & $4.840 \mathrm{E}-14$ \\
\hline $5 F 0410.03$ & $970^{\circ} \mathrm{C}$ & 0.73252 & 0.03028 & 0.86882 & 0.00039 & 4.229460 & 1.00051528 & $5.214 \mathrm{E}-14$ \\
\hline $5 F 0410.04$ & $1020^{\circ} \mathrm{C}$ & 0.75457 & 0.02310 & 0.69109 & 0.00038 & 4.231433 & 1.00051545 & 4.147E-14 \\
\hline $5 F 0410.05$ & $1070^{\circ} \mathrm{C}$ & 0.73831 & 0.02287 & 0.70741 & 0.00029 & 4.233349 & 1.00051561 & 4.246E-14 \\
\hline $5 F 0410.06$ & $1120^{\circ} \mathrm{C}$ & 0.75373 & 0.02383 & 0.65818 & 0.00028 & 4.235323 & 1.00051578 & 3.950E-14 \\
\hline $5 F 0410.07$ & $1190^{\circ} \mathrm{C}$ & $\begin{array}{l}0.74745 \\
072932\end{array}$ & $\begin{array}{l}0.01485 \\
0.019655\end{array}$ & 0.65145 & 0.00031 & $\begin{array}{r}4.237299 \\
4.2329019\end{array}$ & $\begin{array}{l}1.00051595 \\
1\end{array}$ & $3.910 E-14$ \\
\hline $\begin{array}{l}5 F 0440.08 \\
5 F 0410.09\end{array}$ & $1370^{\circ} \mathrm{C}$ & $\begin{array}{l}0.12932 \\
0.75923\end{array}$ & $\begin{array}{l}0.01965 \\
0.02882\end{array}$ & $\begin{array}{l}0.599981 \\
0.5091\end{array}$ & $\begin{array}{l}0.00466 \\
0.00032\end{array}$ & 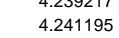 & $\begin{array}{l}1.0000516167 \\
1.0051627\end{array}$ & $\begin{array}{l}3.596 \mathrm{E}-14 \\
3.059 \mathrm{E}-14\end{array}$ \\
\hline $5 F 0410.10$ & $1670^{\circ} \mathrm{C}$ & 1.02605 & 0.13258 & 1.01285 & 0.00031 & 4.243115 & 1.00051644 & $6.077 \mathrm{E}-14$ \\
\hline
\end{tabular}


Age Plateau 


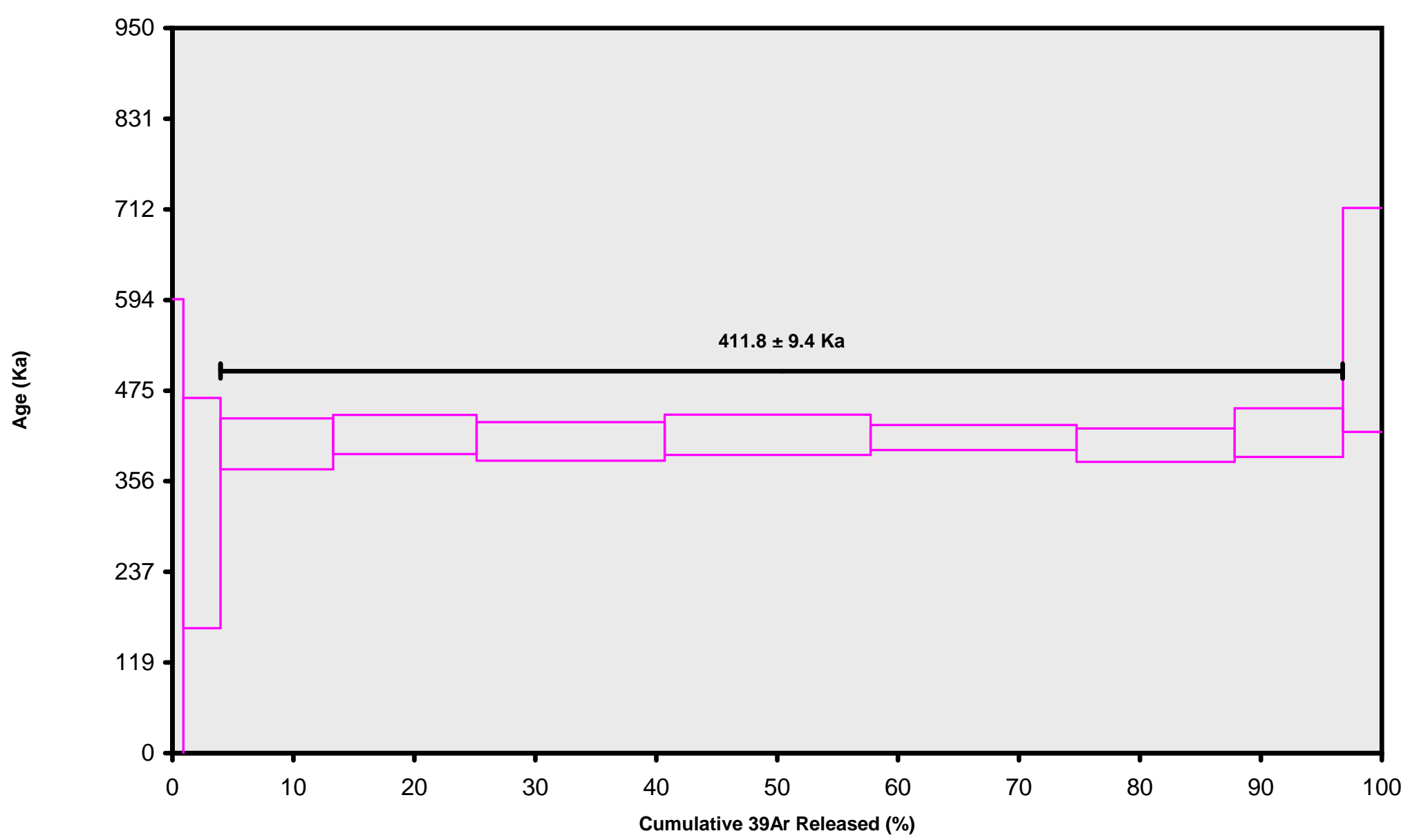

\section{Ar-Ages in $\mathrm{Ka}$}

Weighted Plateau

$411.8 \pm 9.4$

Total Fusion

$412.3 \pm 11.8$

Normal Isochron

$413.6 \pm 19.2$

Inverse Isochron

$413.8 \pm 19.2$

\section{MSWD}

0.24

Sample Info

bas grndmss

Westaway Volcanics

$\mathrm{msp}$

cl155-

$0.0003066(J)$ 
K-Ca Plateau 


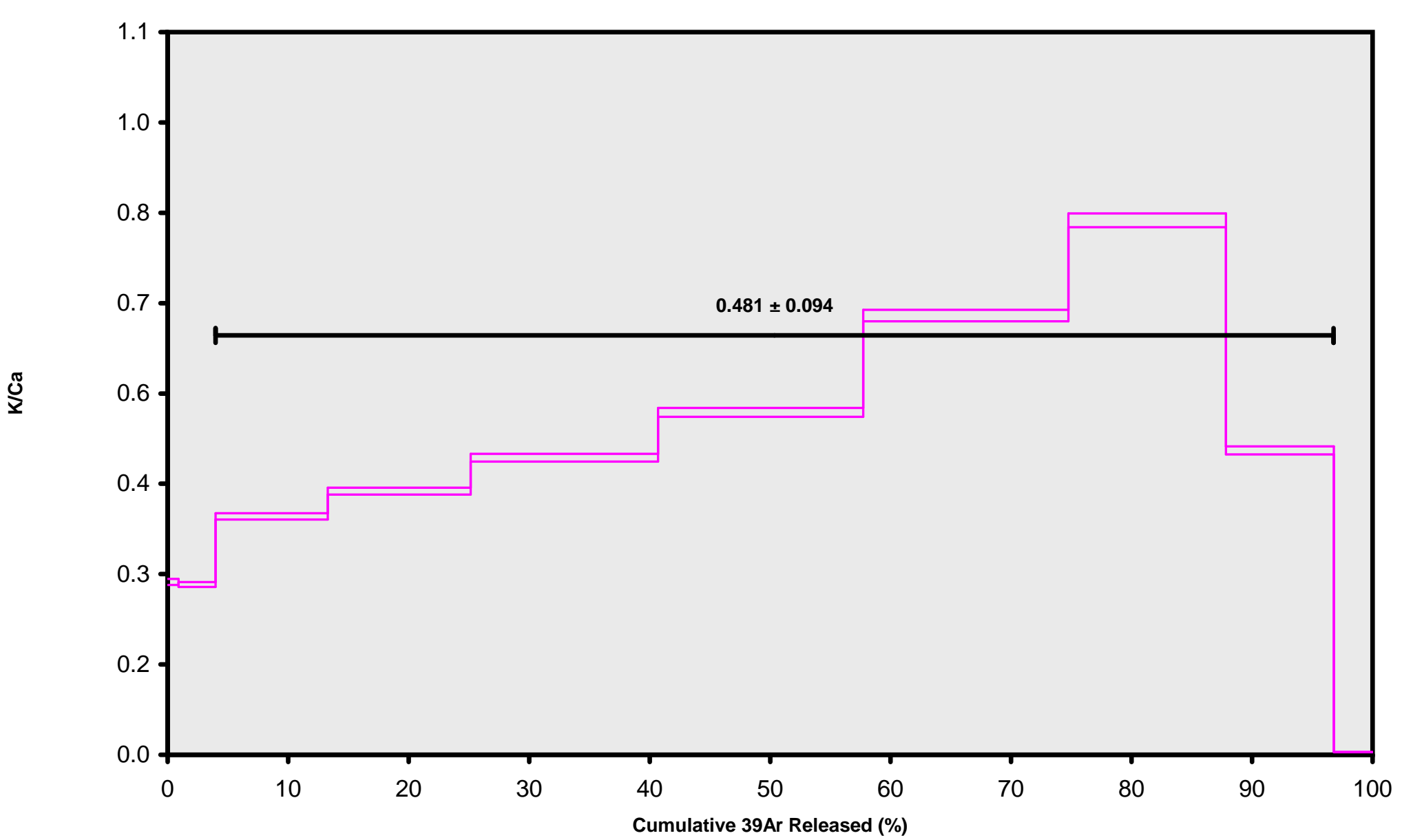

\section{Ar-Ages in $\mathrm{Ka}$}

Weighted Plateau

$411.8 \pm 9.4$

Total Fusion

$412.3 \pm 11.8$

Normal Isochron

$413.6 \pm 19.2$

Inverse Isochron

$413.8 \pm 19.2$ 
Normal Isochron 


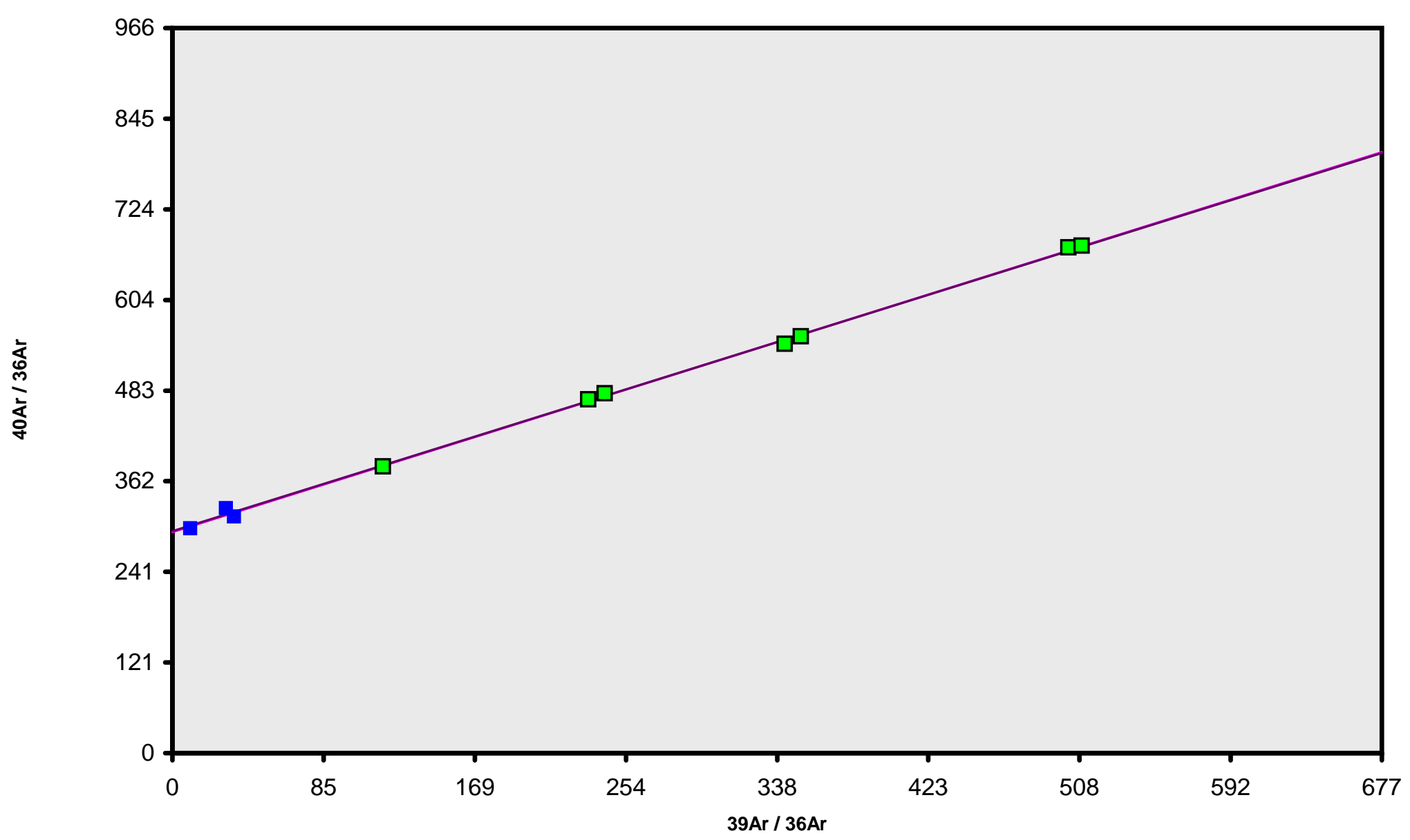

\section{Ar-Ages in $\mathrm{Ka}$}

Weighted Plateau

$411.8 \pm 9.4$

Total Fusion

$412.3 \pm 11.8$

Normal Isochron

$413.6 \pm 19.2$

Inverse Isochron

$413.8 \pm 19.2$

\section{MSWD}

0.28

Sample Info

bas grndmss

Westaway Volcanics

$\mathrm{msp}$

cl155-

$0.0003066(\mathrm{~J})$ 


\section{Inverse Isochron}




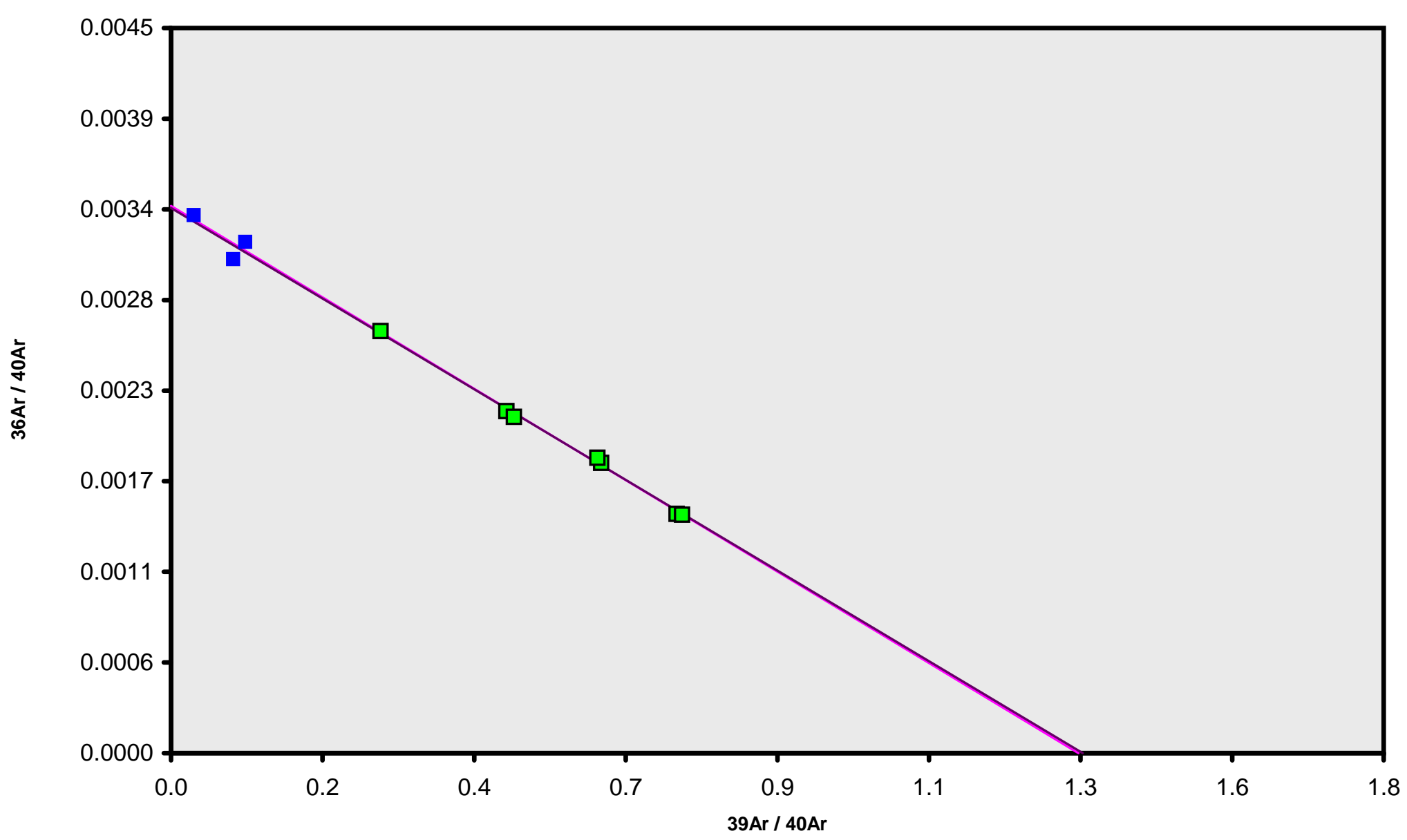

\section{Ar-Ages in $\mathrm{Ka}$}

Weighted Plateau

$411.8 \pm 9.4$

Total Fusion

$412.3 \pm 11.8$

Normal Isochron

$413.6 \pm 19.2$

Inverse Isochron

$413.8 \pm 19.2$

\section{MSWD}

0.28

Sample Info

bas grndmss

Westaway Volcanics

msp

cl155-

$0.0003066(J)$ 
Sample 01TR47 
Data Tables 
MIT, Cambridge, USA

\begin{tabular}{|c|c|c|c|c|c|c|c|c|c|c|c|}
\hline \multicolumn{2}{|c|}{$\begin{array}{l}\text { Procedure } \\
\text { Blanks }\end{array}$} & $36 \mathrm{Ar}$ & $1 \sigma$ & $37 \mathrm{Ar}$ & $1 \sigma$ & $38 \mathrm{Ar}$ & $1 \sigma$ & $39 \mathrm{Ar}$ & $1 \sigma$ & $40 \mathrm{Ar}$ & $1 \sigma$ \\
\hline 5F041N.01 & $\begin{array}{l}770^{\circ} \mathrm{C} \\
8700^{\circ} \mathrm{C}\end{array}$ & 0.000235 & 0.000017 & 0.000226 & 0.000029 & 0.000132 & 0.000023 & 0.000406 & 0.000045 & 0.040000 & 0.000125 \\
\hline $\begin{array}{l}5 F 041 N .02 \\
5 F 04 N \text {. }\end{array}$ & $\begin{array}{l}870^{\circ} \mathrm{C} \\
970^{\circ} \mathrm{C}\end{array}$ & $\begin{array}{l}0.000236 \\
0.000236\end{array}$ & $\begin{array}{l}0.000017 \\
0.000017\end{array}$ & $\begin{array}{l}0.0000227 \\
0.000228\end{array}$ & $\begin{array}{l}0.000029 \\
0.00029\end{array}$ & $\begin{array}{l}0.000132 \\
0.00132\end{array}$ & $\begin{array}{l}0.000023 \\
0.00023\end{array}$ & $\begin{array}{l}0.000408 \\
0.00408\end{array}$ & $\begin{array}{l}0.000045 \\
0.000045\end{array}$ & $\begin{array}{l}0.040000 \\
0.040000\end{array}$ & $\begin{array}{l}0.000125 \\
\end{array}$ \\
\hline 5F041N.04 & $1020^{\circ} \mathrm{C}$ & 0.000236 & 0.000017 & 0.000229 & 0.000029 & 0.000133 & 0.000023 & $\begin{array}{l}0.000409 \\
0.00\end{array}$ & $\begin{array}{l}0.000045 \\
0.000045\end{array}$ & $\begin{array}{l}0.040000 \\
0.040000\end{array}$ & $\begin{array}{l}.00000125 \\
0.000125\end{array}$ \\
\hline $5 F 041$ N. 05 & $1070^{\circ} \mathrm{C}$ & 0.000237 & 0.000017 & 0.000229 & 0.000029 & 0.000133 & 0.000023 & 0.000410 & 0.000045 & 0.040000 & 0.000125 \\
\hline $5 F 041$ N. 06 & $1120^{\circ} \mathrm{C}$ & 0.000237 & 0.000017 & 0.000230 & 0.000029 & 0.000133 & 0.000023 & 0.000410 & 0.000045 & 0.040000 & 0.000125 \\
\hline $5 F 041$ N.07 & $1190^{\circ} \mathrm{C}$ & 0.000237 & 0.000017 & 0.000230 & 0.000029 & 0.000133 & 0.000023 & 0.000411 & 0.000045 & 0.040000 & 0.000125 \\
\hline $5 F 041 N .08$ & $1270^{\circ} \mathrm{C}$ & 0.000237 & 0.000017 & 0.000231 & 0.000029 & 0.000134 & 0.000023 & 0.000412 & 0.000045 & 0.040000 & 0.000125 \\
\hline $\begin{array}{l}5 F 041 N .09 \\
5 F 04 N .1\end{array}$ & $\begin{array}{l}3770^{\circ} \mathrm{C} \\
1670^{\circ} \mathrm{C}\end{array}$ & $\begin{array}{l}0.000238 \\
0.000238\end{array}$ & $\begin{array}{l}0.0000017 \\
0.000017\end{array}$ & $\begin{array}{l}0.0000232 \\
0.000232\end{array}$ & $\begin{array}{l}0.000029 \\
0.000029\end{array}$ & $\begin{array}{l}0.000134 \\
0.000134\end{array}$ & $\begin{array}{l}0.000023 \\
0.000023\end{array}$ & $\begin{array}{l}0.000413 \\
0.000413\end{array}$ & $\begin{array}{l}0.000045 \\
0.000045\end{array}$ & $\begin{array}{l}0.0400000 \\
0.040000\end{array}$ & $\begin{array}{l}0.0000125 \\
0.0000125\end{array}$ \\
\hline
\end{tabular}




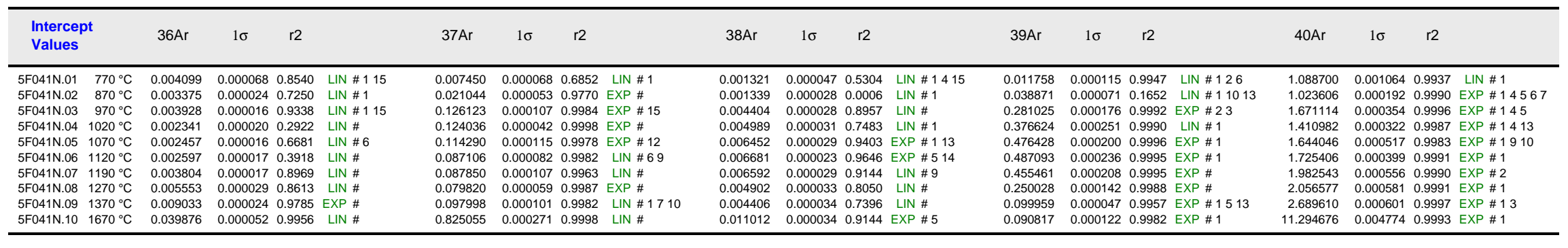


MIT, Cambridge, USA

\begin{tabular}{|c|c|c|c|c|c|c|c|c|c|c|c|c|c|c|c|c|c|c|c|c|c|c|c|}
\hline $\begin{array}{l}\text { Sample } \\
\text { Paramet }\end{array}$ & & Sample & Material & Location & Analyst & 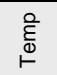 & $\begin{array}{l}\text { Standard } \\
\text { (in Ma) }\end{array}$ & $\% 1 \sigma$ & $\mathrm{J}$ & $\% 1 \sigma$ & Fract & $\% 1 \sigma$ & $\begin{array}{l}\text { Volume } \\
\text { Corr. }\end{array}$ & $\begin{array}{c}\text { Sensitivity } \\
(\mathrm{mol} / \mathrm{vol})\end{array}$ & ฮิ & 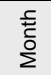 & 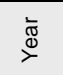 & 호 & $\sum$ & 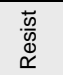 & Irradiation & Project & $\begin{array}{l}\text { Standard } \\
\text { Name }\end{array}$ \\
\hline $\begin{array}{l}\text { 5F041N.01 } \\
\text { 5F041N.02 }\end{array}$ & $\begin{array}{l}770^{\circ} \mathrm{C} \\
870^{\circ} \mathrm{C}\end{array}$ & $\begin{array}{l}01 \mathrm{TR} 47 \mathrm{~B} 18 \\
01 \text { TR47 B18 }\end{array}$ & $\begin{array}{l}\text { bas grndmss } \\
\text { bas grndmss }\end{array}$ & $\begin{array}{l}\text { lestaway Volcanii } \\
\text { Iestaway Volcani }\end{array}$ & $\begin{array}{l}\mathrm{msp} \\
\mathrm{msp}\end{array}$ & $\begin{array}{l}770 \\
870\end{array}$ & $\begin{array}{l}28.34 \\
28.34\end{array}$ & $\begin{array}{l}0.01 \\
0.01\end{array}$ & $\begin{array}{l}0.0003103 \\
0.0003103\end{array}$ & $\begin{array}{l}0.3 \\
0.3\end{array}$ & $\begin{array}{l}1.007 \\
1.007\end{array}$ & $\begin{array}{l}0.2 \\
0.2\end{array}$ & $\begin{array}{l}1 \\
1\end{array}$ & $\begin{array}{l}6.000 E-14 \\
6.000 E-14\end{array}$ & $\begin{array}{l}29 \\
29\end{array}$ & $\begin{array}{l}07 \\
07\end{array}$ & $\begin{array}{l}2005 \\
2005\end{array}$ & $\begin{array}{l}14 \\
15\end{array}$ & $\begin{array}{l}16 \\
36\end{array}$ & $\begin{array}{l}001 \\
001\end{array}$ & $\begin{array}{l}\text { cl155- } \\
\text { cl155- }\end{array}$ & $\begin{array}{l}\text { Westaway } \\
\text { Westaway }\end{array}$ & $\begin{array}{l}\text { tcr-2a } \\
\text { tcr-2a }\end{array}$ \\
\hline $\begin{array}{l}5 F 041 N .03 \\
5\end{array}$ & $970^{\circ} \mathrm{C}$ & 01 TR47 B18 & bas grndmss & lestaway Volcanit & $\mathrm{msp}$ & 970 & 28.34 & 0.01 & 0.0003103 & 0.3 & 1.007 & 0.2 & 1 & $6.000 E-14$ & 29 & 07 & 2005 & 16 & 09 & 001 & cl155- & Westaway & tor-2a \\
\hline 5 F041N.04 & $1020^{\circ} \mathrm{C}$ & 01 TR47 B18 & bas grndmss & lestaway Volcanii & msp & 1020 & 28.34 & 0.01 & 0.0003103 & 0.3 & 1.007 & 0.2 & 1 & $6.000 \mathrm{E}-14$ & 29 & 07 & 2005 & 16 & 43 & 001 & & Westaway & tcr-2a \\
\hline $5 F 041 \mathrm{~N} .05$ & $1070^{\circ} \mathrm{C}$ & 01TR47 B18 & bas grndmss & Iestaway Volcanii & msp & 1070 & 28.34 & 0.01 & 0.0003103 & 0.3 & 1.007 & 0.2 & 1 & $6.000 E-14$ & 29 & 07 & 2005 & 17 & 16 & 001 & & Westaway & tcr-2a \\
\hline $5 F 041 \mathrm{~N} .06$ & $1120^{\circ} \mathrm{C}$ & 01TR47 B18 & bas grndmss & Iestaway Volcani & $\mathrm{msp}$ & 1120 & 28.34 & 0.01 & 0.0003103 & 0.3 & 1.007 & 0.2 & 1 & $6.000 \mathrm{E}-14$ & 29 & 07 & 2005 & 17 & 50 & 001 & cl155- & Westaway & tcr-2a \\
\hline $\begin{array}{l}5 F 041 N .07 \\
5 F 41 N 08\end{array}$ & $\begin{array}{ll}1190^{\circ} \mathrm{C} \\
1270\end{array}$ & $\begin{array}{l}01 T R 47 \text { B18 } \\
017 \text { R }\end{array}$ & bas grndmss & Iestaway Volcani & msp & $\begin{array}{l}1190 \\
1270\end{array}$ & 28.34 & 0.01 & 0.0003103 & 0.3 & 1.007 & 0.2 & 1 & $6.000 \mathrm{E}-14$ & 29 & 07 & 2005 & 18 & 23 & 001 & & Westaway & tcr-2a \\
\hline $\begin{array}{l}\text { SFF41N.08 } \\
\text { 5F041N.09 }\end{array}$ & $\begin{array}{l}1270{ }^{\circ} \mathrm{C} \\
1370^{\circ} \mathrm{C}\end{array}$ & 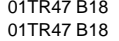 & $\begin{array}{l}\text { bas grndmss } \\
\text { bas grndmss }\end{array}$ & $\begin{array}{l}\text { lestaway Volcanii } \\
\text { lestaway Volcanii }\end{array}$ & $\begin{array}{l}\mathrm{msp} \\
\mathrm{msp}\end{array}$ & $\begin{array}{l}1270 \\
1370\end{array}$ & $\begin{array}{l}28.34 \\
28.34\end{array}$ & $\begin{array}{l}0.01 \\
0.01\end{array}$ & $\begin{array}{l}0.0003103 \\
0.0003103\end{array}$ & $\begin{array}{l}0.3 \\
0.3\end{array}$ & $\begin{array}{l}1.007 \\
1.007\end{array}$ & $\begin{array}{l}0.2 \\
0.2\end{array}$ & $\begin{array}{l}1 \\
1\end{array}$ & $\begin{array}{l}6.000 \mathrm{E}-14 \\
6.000 \mathrm{E}-14\end{array}$ & $\begin{array}{l}29 \\
29\end{array}$ & $\begin{array}{l}07 \\
07\end{array}$ & $\begin{array}{l}2005 \\
2005\end{array}$ & $\begin{array}{l}18 \\
19\end{array}$ & $\begin{array}{l}57 \\
30\end{array}$ & $\begin{array}{l}001 \\
001\end{array}$ & $\begin{array}{l}\text { Cll55- } \\
\text { cl155- }\end{array}$ & $\begin{array}{l}\text { Westaway } \\
\text { Westaway }\end{array}$ & $\begin{array}{l}\text { trc-2a } \\
\text { tcr-2a }\end{array}$ \\
\hline 5F041N.10 & $1670^{\circ} \mathrm{C}$ & 01 TR47 B18 & bas grndmss & Iestaway Volcani & msp & 1670 & 28.34 & 0.01 & 0.0003103 & 0.3 & 1.007 & 0.2 & 1 & $6.000 \mathrm{E}-14$ & 29 & 07 & 2005 & 20 & 04 & 001 & cl155- & Westaway & tcr-2a \\
\hline
\end{tabular}




\begin{tabular}{|c|c|c|c|c|c|c|c|c|c|c|c|c|c|c|c|c|c|c|c|c|c|c|c|}
\hline $\begin{array}{l}\text { Irradiati } \\
\text { Constar }\end{array}$ & & 40/36(a) & $\% 1 \sigma$ & 38/36(a) & $\% 1 \sigma$ & 39/37(ca) & $\% 1 \sigma$ & $38 / 37$ (са) & $\% 1 \sigma$ & 36/37(ca) & $\% 1 \sigma$ & $40 / 39(\mathrm{k})$ & $\% 1 \sigma$ & $38 / 39(k)$ & $\% 1 \sigma$ & 36/38(cl) & $\% 1 \sigma$ & $\mathrm{K} / \mathrm{Ca}$ & $\% 1 \sigma$ & $\mathrm{K} / \mathrm{Cl}$ & $\% 1 \sigma$ & $\mathrm{Ca} / \mathrm{Cl}$ & $\% 1 \sigma$ \\
\hline $5 F 041 \mathrm{~N} .01$ & $770^{\circ} \mathrm{C}$ & 295.5 & 0 & 0.1869 & 0 & 0.000676 & 0 & 0.000034 & 0 & 0.000279 & 0 & 0.00039 & 0 & 0.01243 & 0 & 0 & 0 & 0.49 & 0 & 0 & 0 & 0 & 0 \\
\hline 5F041N.02 & $870^{\circ} \mathrm{C}$ & 295.5 & 0 & 0.1869 & 0 & 0.000676 & 0 & 0.000034 & 0 & 0.000279 & 0 & 0.00039 & 0 & 0.01243 & 0 & 0 & 0 & 0.49 & 0 & 0 & 0 & 0 & 0 \\
\hline $5 F 041 \mathrm{~N} .03$ & $970^{\circ} \mathrm{C}$ & 295.5 & 0 & 0.1869 & 0 & 0.000676 & 0 & 0.000034 & 0 & 0.000279 & 0 & 0.00039 & 0 & 0.01243 & 0 & 0 & 0 & 0.49 & 0 & 0 & 0 & 0 & 0 \\
\hline $5 F 041 N .04$ & $1020^{\circ} \mathrm{C}$ & 295.5 & 0 & & 0 & 0.000676 & 0 & 0.000034 & 0 & 0.000279 & 0 & 0.00039 & 0 & 0.01243 & 0 & 0 & 0 & 0.49 & 0 & 0 & 0 & 0 & 0 \\
\hline 5F041N.05 & $1070^{\circ} \mathrm{C}$ & $\begin{array}{l}295.5 \\
205.5\end{array}$ & 0 & 0.1869 & 0 & 0.000676 & 0 & 0.000034 & 0 & 0.000279 & 0 & 0.00039 & 0 & 0.01243 & 0 & 0 & 0 & 0.49 & 0 & 0 & 0 & 0 & 0 \\
\hline $\begin{array}{l}5 F 044 \mathrm{~N} .06 \\
5 \mathrm{~F} 041 \mathrm{~N} 07\end{array}$ & $\begin{array}{l}1120^{\circ} \mathrm{C} \\
1190^{\circ} \mathrm{C}\end{array}$ & $\begin{array}{r}295.5 \\
295.5\end{array}$ & $\begin{array}{l}0 \\
0\end{array}$ & $\begin{array}{l}0.1869 \\
0.1669\end{array}$ & $\begin{array}{l}0 \\
0\end{array}$ & $\begin{array}{l}0.000676 \\
0\end{array}$ & $\begin{array}{l}0 \\
0\end{array}$ & $\begin{array}{l}0.000034 \\
0.000034\end{array}$ & $\begin{array}{l}0 \\
0\end{array}$ & $\begin{array}{l}0.000279 \\
0.00279\end{array}$ & 0 & $\begin{array}{l}0.0000399 \\
0.0039\end{array}$ & 0 & 0.01243 & 0 & 0 & 0 & 0.49 & ${ }^{0}$ & 0 & 0 & 0 & 0 \\
\hline $\begin{array}{l}5 F 041 N .0 / \\
\text { 5F041N. }\end{array}$ & $1270^{\circ} \mathrm{C}$ & $\begin{array}{l}295.5 \\
295.5\end{array}$ & $\begin{array}{l}0 \\
0\end{array}$ & $\begin{array}{l}0.1869 \\
0.1869\end{array}$ & $\begin{array}{l}0 \\
0\end{array}$ & $\begin{array}{l}0.0006 / 6 \\
0.000676\end{array}$ & $\begin{array}{l}0 \\
0\end{array}$ & $\begin{array}{l}0.000034 \\
0.000034\end{array}$ & 0 & $\begin{array}{l}0.000279 \\
0.00279\end{array}$ & 0 & $\begin{array}{l}0.0000039 \\
0.00039\end{array}$ & 0 & $\begin{array}{l}0.01243 \\
0.01243\end{array}$ & 0 & 0 & 0 & 0.49 & 0 & 0 & 0 & 0 & $\begin{array}{l}0 \\
0 \\
0\end{array}$ \\
\hline 5F041N.09 & $1370^{\circ} \mathrm{C}$ & 295.5 & 0 & 0.1869 & 0 & 0.000676 & 0 & 0.000034 & 0 & 0.000279 & 0 & 0.00039 & 0 & 0.01243 & 0 & 0 & 0 & 0.49 & 0 & 0 & 0 & 0 & 0 \\
\hline 5F041N.10 & $1670^{\circ} \mathrm{C}$ & 295.5 & 0 & 0.1869 & 0 & 0.000676 & 0 & 0.000034 & 0 & 0.000279 & 0 & 0.00039 & 0 & 0.01243 & 0 & 0 & 0 & 0.49 & 0 & 0 & 0 & 0 & 0 \\
\hline
\end{tabular}




\begin{tabular}{|c|c|c|c|c|c|c|c|c|c|c|}
\hline \multicolumn{2}{|c|}{$\begin{array}{l}\text { Incremental } \\
\text { Heating }\end{array}$} & \multirow{2}{*}{$\begin{array}{r}36 \operatorname{Ar}(a) \\
0.00375\end{array}$} & \multirow{2}{*}{$\begin{array}{r}37 \mathrm{Ar}(\mathrm{ca}) \\
0.02971\end{array}$} & $38 \mathrm{Ar}(\mathrm{cl})$ & $39 \mathrm{Ar}(\mathrm{k})$ & 40Ar(r) & $\begin{array}{l}\mathrm{Age} \pm 2 \sigma \\
\text { (Ma) }\end{array}$ & \multicolumn{2}{|c|}{$\begin{array}{l}40 \operatorname{Ar}(r) 39 \operatorname{Ar}(k) \\
(\%) \quad(\%)\end{array}$} & $\mathrm{K} / \mathrm{Ca} \pm 2 \sigma$ \\
\hline 5 F041N.01 & $770{ }^{\circ} \mathrm{C}$ & & & 0.00033 & 0.01126 & 0.00000 & $0.0 \pm 0.0$ & 0.00 & 0.44 & $0.186 \pm 0.006$ \\
\hline $\begin{array}{l}5 F 041 N .02 \\
5 F 04 N 03\end{array}$ & $870^{\circ} \mathrm{C}$ & 0.00303 & 0.08571 & 0.00015 & 0.03816 & 0.08825 & $1294.2 \pm 321.6$ & 8.97 & 1.50 & $0.218 \pm 0.003$ \\
\hline $\begin{array}{l}\text { FF041N.03 } \\
\text { 5F041N.04 }\end{array}$ & $\begin{array}{r}970^{\circ} \mathrm{C} C \\
102{ }^{\circ} \mathrm{C} \\
\quad 4\end{array}$ & $\begin{array}{l}0.00345 \\
0.00190\end{array}$ & $\begin{array}{l}0.551856 \\
0.51020\end{array}$ & $\begin{array}{l}0.00009 \\
0.00000\end{array}$ & $\begin{array}{l}0.27846 \\
0.37345\end{array}$ & $\begin{array}{l}0.61257 \\
0.80794\end{array}$ & $\begin{array}{l}1231.1 \pm 43.4 \\
1210.7 \pm \pm 27.3\end{array}$ & $\begin{array}{l}37.56 \\
58.93\end{array}$ & $\begin{array}{l}10.95 \\
14.68\end{array}$ & $\begin{array}{l}0.263 \pm 0.003 \\
0.359 \pm 0.005\end{array}$ \\
\hline 5F041N.05 & $1070^{\circ} \mathrm{C}$ & 0.00203 & 0.47025 & 0.00000 & 0.47263 & 1.00436 & $1189.2 \pm 20.5$ & 62.61 & 18.59 & $0.492 \pm 0.006$ \\
\hline 5F041N.06 & $1120^{\circ} \mathrm{C} \quad 4$ & 0.00220 & 0.35834 & 0.00003 & 0.48331 & 1.03627 & $1199.9 \pm 20.6$ & 61.48 & 19.00 & $0.661 \pm 0.008$ \\
\hline 5F041N.07 & $1190^{\circ} \mathrm{C} \quad 4$ & 0.00337 & 0.36157 & 0.00011 & 0.45187 & 0.94682 & $1172.6 \pm 26.8$ & 48.74 & 17.77 & $0.612 \pm 0.008$ \\
\hline $5 F 041 N .08$ & $1270^{\circ} \mathrm{C}$ & 0.00508 & 0.32858 & 0.00066 & 0.24779 & 0.51571 & $1164.7 \pm 70.0$ & 25.57 & 9.74 & $0.370 \pm 0.005$ \\
\hline \multirow[t]{2}{*}{$\begin{array}{l}5 F 041 \text { N.09 } \\
\text { 5F041N.10 }\end{array}$} & $\begin{array}{l}1370^{\circ} \mathrm{C} \\
1670^{\circ} \mathrm{C}\end{array}$ & $\begin{array}{l}0.00844 \\
0.03761\end{array}$ & $\begin{array}{l}0.40381 \\
3.40842\end{array}$ & $\begin{array}{l}0.00139 \\
0.00249\end{array}$ & $\begin{array}{l}0.09863 \\
0.08752\end{array}$ & $\begin{array}{l}0.15461 \\
0.14154\end{array}$ & $\begin{array}{l}877.3 \pm 244.8 \\
905.1+1160.9\end{array}$ & $\begin{array}{l}5.84 \\
1.26\end{array}$ & $\begin{array}{l}3.88 \\
3.44\end{array}$ & $\begin{array}{l}0.120 \pm 0.002 \\
0.013+0.000\end{array}$ \\
\hline & $\Sigma$ & 0.07085 & 6.47516 & 0.00526 & 2.54307 & 5.30806 & & & & \\
\hline \multicolumn{2}{|c|}{$\begin{array}{l}\text { Information } \\
\text { on Analysis }\end{array}$} & & \multicolumn{2}{|c|}{ Results } & \multicolumn{2}{|c|}{$40(r) / 39(k) \pm 2 \sigma$} & $\begin{array}{l}\text { Age } \pm 2 \sigma \\
\text { (Ma) }\end{array}$ & $\sum_{\substack{0 \\
\text { Wn }}}^{0}$ & $\begin{array}{l}39 \mathrm{Ar}(\mathrm{k}) \\
(\%, \mathrm{n})\end{array}$ & $\mathrm{K} / \mathrm{Ca} \pm 2 \sigma$ \\
\hline \multirow{2}{*}{$\begin{array}{l}\text { Sample } \\
\text { Material } \\
\text { Location } \\
\text { Analyst }\end{array}$} & \multirow{2}{*}{$\begin{array}{c}01 \text { TR47 B18 } \\
\text { bas grndmss } \\
\text { estaway Volcan } \\
\text { msp }\end{array}$} & & \multirow{2}{*}{\multicolumn{2}{|c|}{ Weighted Plateau }} & \multirow[t]{2}{*}{2.1355} & $\begin{array}{l} \pm 0.0252 \\
\pm 1180 \%\end{array}$ & $1195.1 \pm 15.8$ & \multirow{2}{*}{$\begin{array}{c}1.64 \\
2.57 \\
1.2789\end{array}$} & \multirow{2}{*}{$\begin{array}{c}90.74 \\
6 \\
6 \\
\text { Statistica }\end{array}$} & $0.374 \pm 0.109$ \\
\hline & & & & & & \multicolumn{2}{|c|}{$\begin{array}{c}=1.18 \% \text { External Error } \pm 1.32 \% \\
\pm 15.8 \\
\text { Analytical Error } \pm 14.1\end{array}$} & & & \\
\hline $\begin{array}{l}\text { Project } \\
\text { Irradiation } \\
\text { J-value } \\
\text { Standard }\end{array}$ & $\begin{array}{c}\text { Westaway } \\
\text { cl1155- } \\
0.0003103 \\
28.34\end{array}$ & & \multicolumn{2}{|c|}{ Total Fusion Age } & 2.0873 & $\begin{array}{r} \pm 0.0768 \\
\pm 3.68 \% \\
\text { Ex } \\
\text { Ana }\end{array}$ & $\begin{aligned} & 1168.1 \pm 43.5 \\
& \pm 3.73 \% \\
& \text { nal Error } \pm \pm 3.5 \\
& \text { cal Error } \pm 43.0\end{aligned}$ & & 10 & $0.192 \pm 0.001$ \\
\hline
\end{tabular}


MIT, Cambridge, USA

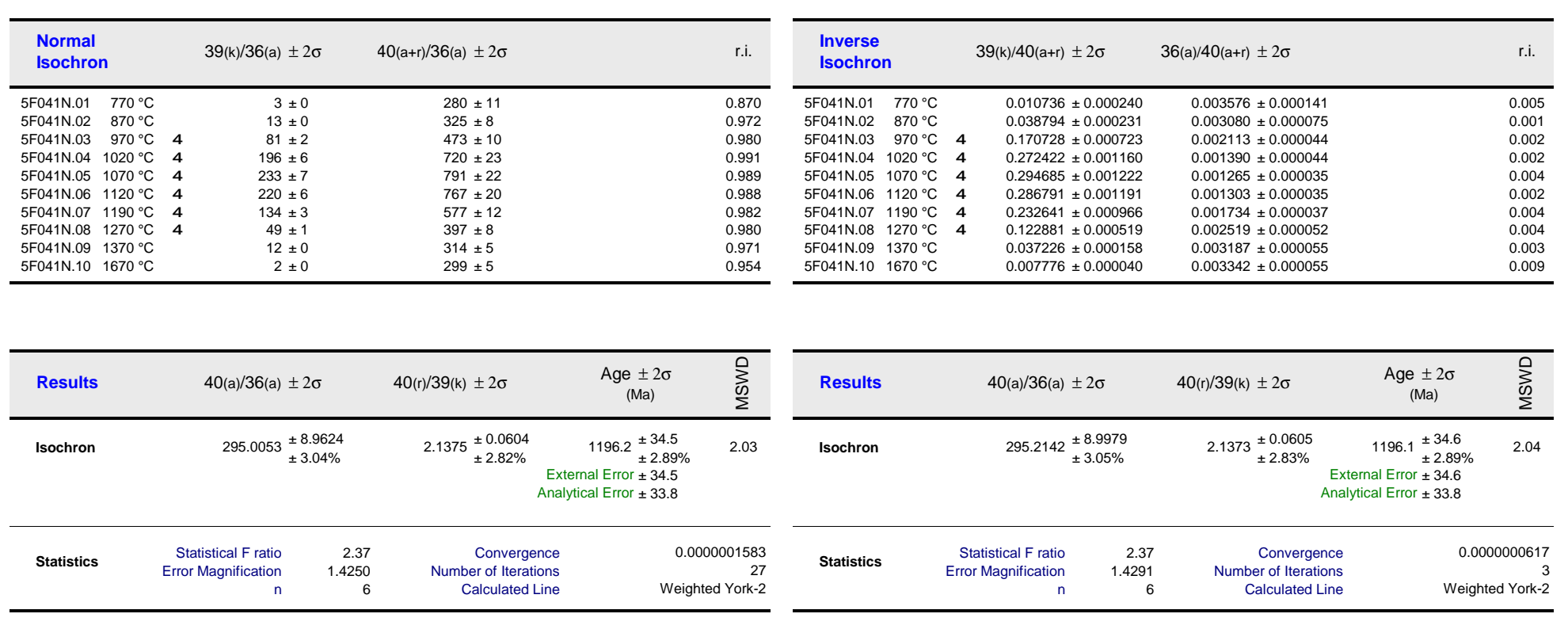




\begin{tabular}{|c|c|c|c|c|c|c|c|c|c|c|c|c|c|c|c|}
\hline \multicolumn{3}{|c|}{$\begin{array}{l}\text { Degassing } \\
\text { Patterns }\end{array}$} & \multirow{2}{*}{$\begin{array}{r}36 \operatorname{Ar}(\mathrm{a}) \\
0.00375 \\
0.00303\end{array}$} & \multirow{2}{*}{$\begin{array}{r}36 \operatorname{Ar}(\mathrm{ca}) \\
0.00001\end{array}$} & \multirow{2}{*}{$\begin{array}{c}36 \operatorname{Ar}(\mathrm{cl}) \\
0.00000 \\
0.00000\end{array}$} & \multirow{2}{*}{$\begin{array}{r}37 \mathrm{Ar}(\mathrm{ca}) \\
0.02971\end{array}$} & \multirow{2}{*}{$\begin{array}{l}38 \mathrm{Ar}(\mathrm{a}) \\
0.00070\end{array}$} & \multirow{2}{*}{$\begin{array}{r}38 \mathrm{Ar}(\mathrm{k}) \\
0.00014\end{array}$} & \multirow{2}{*}{$\begin{array}{r}38 \mathrm{Ar}(\mathrm{ca}) \\
0.00000 \\
0.0000\end{array}$} & \multirow{2}{*}{$\begin{array}{r}38 \mathrm{Ar}(\mathrm{cl}) \\
0.00033\end{array}$} & \multirow{2}{*}{$\begin{array}{r}39 \mathrm{Ar}(\mathrm{k}) \\
0.01126\end{array}$} & \multirow{2}{*}{$\begin{array}{r}39 \operatorname{Ar}(\mathrm{ca}) \\
0.00002\end{array}$} & \multirow{2}{*}{$\begin{array}{l}40 \mathrm{Ar}(\mathrm{r}) \\
0.00000\end{array}$} & \multirow{2}{*}{$\begin{array}{l}40 \operatorname{Ar}(a) \\
1.10814 \\
0.89534\end{array}$} & \multirow{2}{*}{$\begin{array}{r}40 \operatorname{Ar}(\mathrm{k}) \\
0.00000\end{array}$} \\
\hline $\begin{array}{l}5 \mathrm{~F} 041 \mathrm{~N} .01 \\
5 \mathrm{~F} 041 \mathrm{~N} .02\end{array}$ & $\begin{array}{l}770^{\circ} \mathrm{C} \\
870\end{array}$ & & & & & & & & & & & & & & \\
\hline $\begin{array}{l}\text { SFoutN.02 } \\
\text { 5F041N.02 }\end{array}$ & $970^{\circ} \mathrm{C}$ & 4 & $\begin{array}{l}0.003033 \\
0.00345\end{array}$ & $\begin{array}{l}0.00002 \\
0.00014\end{array}$ & $\begin{array}{l}0.00000 \\
0.00000\end{array}$ & $\begin{array}{l}0.08517 \\
0.51856\end{array}$ & $\begin{array}{l}0.0005 / \\
0.00064\end{array}$ & $\begin{array}{l}0.00044 \\
0.00346\end{array}$ & $\begin{array}{l}0.00000 \\
0.00002\end{array}$ & $\begin{array}{l}0.000015 \\
0.00009\end{array}$ & $\begin{array}{l}0.03816 \\
0.27846\end{array}$ & $\begin{array}{l}0.00006 \\
0.00035\end{array}$ & $\begin{array}{l}0.088825 \\
0.61257\end{array}$ & $\begin{array}{l}0.895344 \\
1.01843\end{array}$ & $\begin{array}{l}0.00001 \\
0.00011\end{array}$ \\
\hline 5F041N.04 & $1020^{\circ} \mathrm{C}$ & 4 & 0.00190 & 0.00014 & 0.00000 & 0.51020 & 0.00036 & 0.00464 & 0.00002 & 0.00000 & 0.37345 & 0.00034 & 0.80794 & 0.56289 & $\begin{array}{l}0.00011 \\
0.00015\end{array}$ \\
\hline 5F041N.05 & $1070^{\circ} \mathrm{C}$ & 4 & 0.00203 & 0.00013 & 0.00000 & 0.47025 & 0.00038 & 0.00587 & 0.00002 & 0.000 & 0.47263 & 0.00032 & 00436 & 0.59950 & 0.00 \\
\hline 5F041N.06 & $1120^{\circ} \mathrm{C}$ & 4 & 0.00220 & 0.00010 & 0.00000 & 0.358 & 0.000 & 501 & 0.000 & & 0.48 & & & 0.64895 & 0.00 \\
\hline & $1190^{\circ} \mathrm{C}$ & 4 & 0.00337 & 0.00010 & 0.00000 & 0.361 & 0.00063 & 0.00562 & 0.00001 & & 0.45187 & & 682 & 0.99555 & 0.00 \\
\hline & $1270^{\circ} \mathrm{C}$ & 4 & 0.00508 & 0.0000 & 0.000 & 0.328 & 0.00095 & 0.00308 & 0.000 & & & & & & 0.00010 \\
\hline & & & & & & & & 0.00123 & 0.00001 & & & & & & 0.00004 \\
\hline \multirow[t]{3}{*}{ 5F041N.10 } & $1670^{\circ} \mathrm{C}$ & & 0.03761 & 0.00095 & 0.00000 & 3.40842 & 0.00703 & 0.00109 & 0.00012 & 0.00249 & 0.08752 & 0.00230 & 0.14154 & 11.11311 & 0.00003 \\
\hline & & $\Sigma$ & 0.07085 & 0.00181 & 0.00000 & 6.47516 & 0.01324 & 0.03161 & 0.00022 & 0.00526 & 2.54307 & 0.00438 & 5.30806 & 20.93765 & 0.00099 \\
\hline & & $\Sigma$ & & & & & & & & & & & & & \\
\hline
\end{tabular}

\begin{tabular}{|c|c|c|c|c|c|c|c|c|c|}
\hline \multicolumn{3}{|c|}{$\begin{array}{l}\text { Additional } \\
\text { Ratios }\end{array}$} & \multirow{2}{*}{$\begin{array}{r}40(\mathrm{r}) / 39(\mathrm{k}) \\
0.00000\end{array}$} & \multirow{2}{*}{$\frac{1 \sigma}{0.00000}$} & \multirow{2}{*}{$\begin{array}{l}40(r+a) \\
1.04870\end{array}$} & \multirow{2}{*}{$\frac{1 \sigma}{0.00107}$} & \multirow{3}{*}{$\begin{array}{r}37 \operatorname{Ar} \text { (decay) } \\
4.198997 \\
4.203607\end{array}$} & \multirow{3}{*}{$\begin{array}{c}39 \operatorname{Ar} \text { (decay) } \\
\begin{array}{l}1.00051270 \\
1.00051309\end{array}\end{array}$} & \multirow{2}{*}{$\begin{array}{r}40 \mathrm{Ar} \text { (moles) } \\
6.292 \mathrm{E}-14\end{array}$} \\
\hline $5 F 041 N .01$ & $7700^{\circ} \mathrm{C}$ & & & & & & & & \\
\hline 5F041N.02 & $870^{\circ} \mathrm{C}$ & & & 0.28749 & 0.98359 & 0.00023 & & & $5.902 \mathrm{E}-14$ \\
\hline $5 F 041 \mathrm{~N} .03$ & $970^{\circ} \mathrm{C}$ & 4 & 2.19986 & 0.03877 & 1.63101 & 0.00038 & 4.205510 & 1.00051325 & $9.787 \mathrm{E}-14$ \\
\hline $5 F 041 \mathrm{~N} .04$ & $1020^{\circ} \mathrm{C}$ & 4 & 2.16348 & 0.02438 & 1.37084 & 0.00034 & 4.207472 & 1.00051342 & $8.226 \mathrm{E}-14$ \\
\hline 5 F041N.05 & $1070^{\circ} \mathrm{C}$ & 4 & 2.12502 & 0.01829 & 1.60386 & 0.00053 & 4.209377 & 1.00051358 & $9.624 \mathrm{E}-14$ \\
\hline $5 F 041 N .06$ & $\begin{array}{lll}1120^{\circ} \mathrm{C} \\
110\end{array}$ & 4 & $\begin{array}{l}2.14412 \\
209532\end{array}$ & $\begin{array}{l}0.01843 \\
0.02399\end{array}$ & $\begin{array}{r}1.68522 \\
1.94237\end{array}$ & 0.00042 & $\begin{array}{l}4.211340 \\
\end{array}$ & $\begin{array}{l}1.00051375 \\
1\end{array}$ & $\begin{array}{l}1.011 \mathrm{E}-13 \\
1\end{array}$ \\
\hline $5 F 044 N .07$ & $\begin{array}{l}1190^{\circ} \mathrm{C} \\
127{ }^{\circ} \mathrm{C}\end{array}$ & $\begin{array}{l}4 \\
4 \\
4\end{array}$ & $\begin{array}{l}2.095322 \\
2.08127\end{array}$ & $\begin{array}{l}0.02399 \\
0.06252\end{array}$ & $\begin{array}{l}.94237 \\
2.01648\end{array}$ & $\begin{array}{l}0.000057 \\
0.00059\end{array}$ & $\begin{array}{l}4.213247 \\
4.212212\end{array}$ & $\begin{array}{l}1.000013591 \\
1.00051408\end{array}$ & $\begin{array}{l}1.166 \mathrm{E}-13 \\
1.10 \mathrm{C}\end{array}$ \\
\hline $\begin{array}{l}5 F 041 N .08 \\
5 F 04 N .09\end{array}$ & $\begin{array}{l}1277 \circ^{\circ} \mathrm{C} \\
130{ }^{\circ} \mathrm{C}\end{array}$ & 4 & $\begin{array}{l}2.08127 \\
1.56754\end{array}$ & $\begin{array}{l}0.06252 \\
0.21878\end{array}$ & $\begin{array}{l}2.0 .1648 \\
2.64957\end{array}$ & $\begin{array}{l}0.000059 \\
0.00061\end{array}$ & $\begin{array}{l}4.215212 \\
4.217121\end{array}$ & $\begin{array}{l}1.000051408 \\
1.00051424\end{array}$ & $\begin{array}{l}1.210 \mathrm{E}-13 \\
1.590 \mathrm{E}-13\end{array}$ \\
\hline 5F041N.10 & $1670^{\circ} \mathrm{C}$ & & 1.61724 & 1.03737 & 11.25464 & 0.00478 & 4.219088 & 1.00051441 & $6.753 \mathrm{E}-13$ \\
\hline
\end{tabular}


Age Plateau 


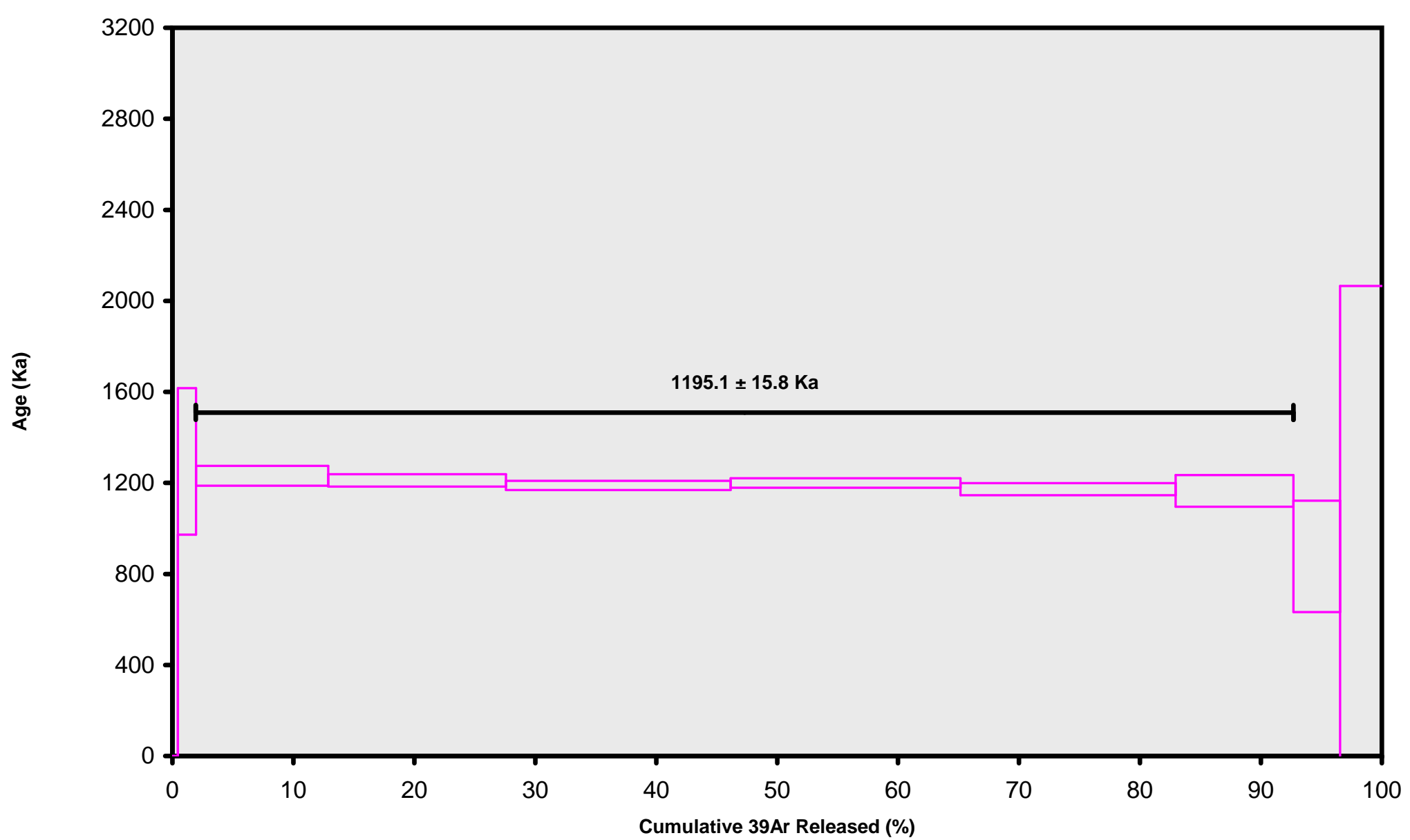

Ar-Ages in $\mathrm{Ka}$

Weighted Plateau

$1195.1 \pm 15.8$

Total Fusion

$1168.1 \pm 43.5$

Normal Isochron

$1196.2 \pm 34.5$

Inverse Isochron

$1196.1 \pm 34.6$

\section{MSWD}

1.64

\section{Sample Info}

bas grndmss

Westaway Volcanics

$\mathrm{msp}$

cl155-

$0.0003103(\mathrm{~J})$ 
K-Ca Plateau 
MIT, Cambridge, USA

\section{RW5F041N.AGE >> 01TR47 B18 >> Westaway}

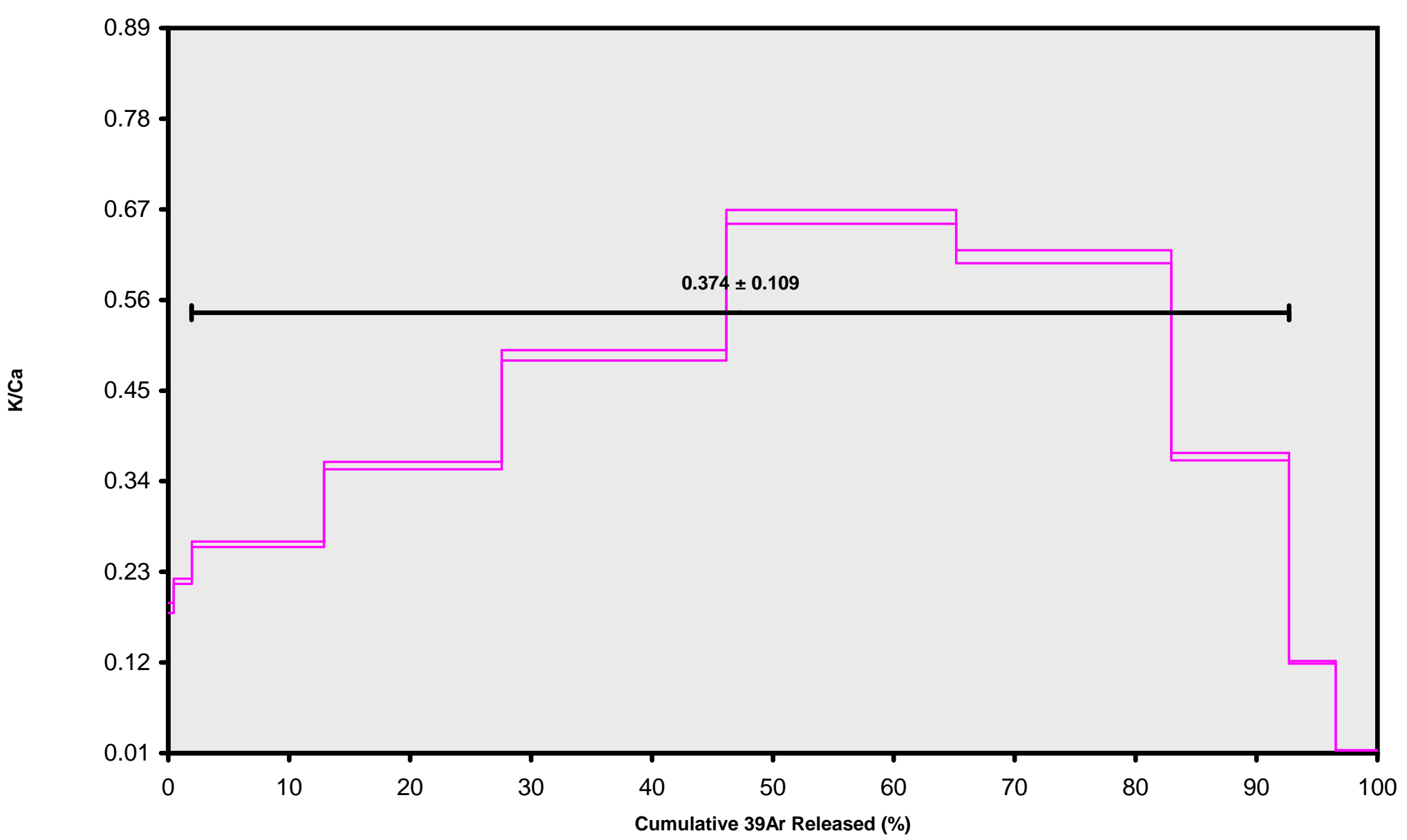

\section{Ar-Ages in $\mathrm{Ka}$}

Weighted Plateau

$1195.1 \pm 15.8$

Total Fusion

$1168.1 \pm 43.5$

Normal Isochron

$1196.2 \pm 34.5$

Inverse Isochron

$1196.1 \pm 34.6$ 
Normal Isochron 


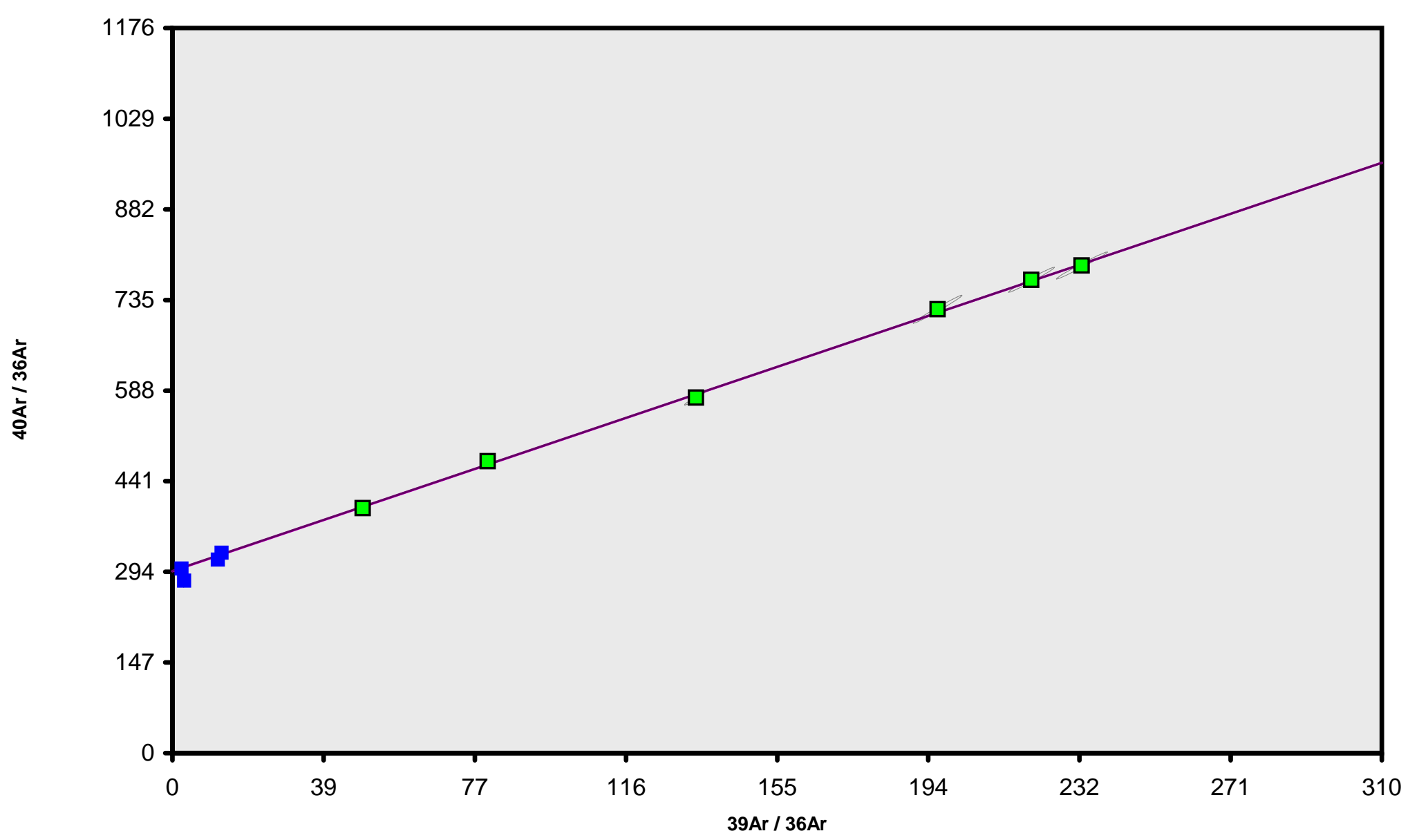

\section{Ar-Ages in $\mathrm{Ka}$}

Weighted Plateau

$1195.1 \pm 15.8$

Total Fusion

$1168.1 \pm 43.5$

Normal Isochron

$1196.2 \pm 34.5$

Inverse Isochron

$1196.1 \pm 34.6$

\section{MSWD}

2.03

Sample Info

bas grndmss

Westaway Volcanics

$\mathrm{msp}$

cl155-

$0.0003103(\mathrm{~J})$ 


\section{Inverse Isochron}




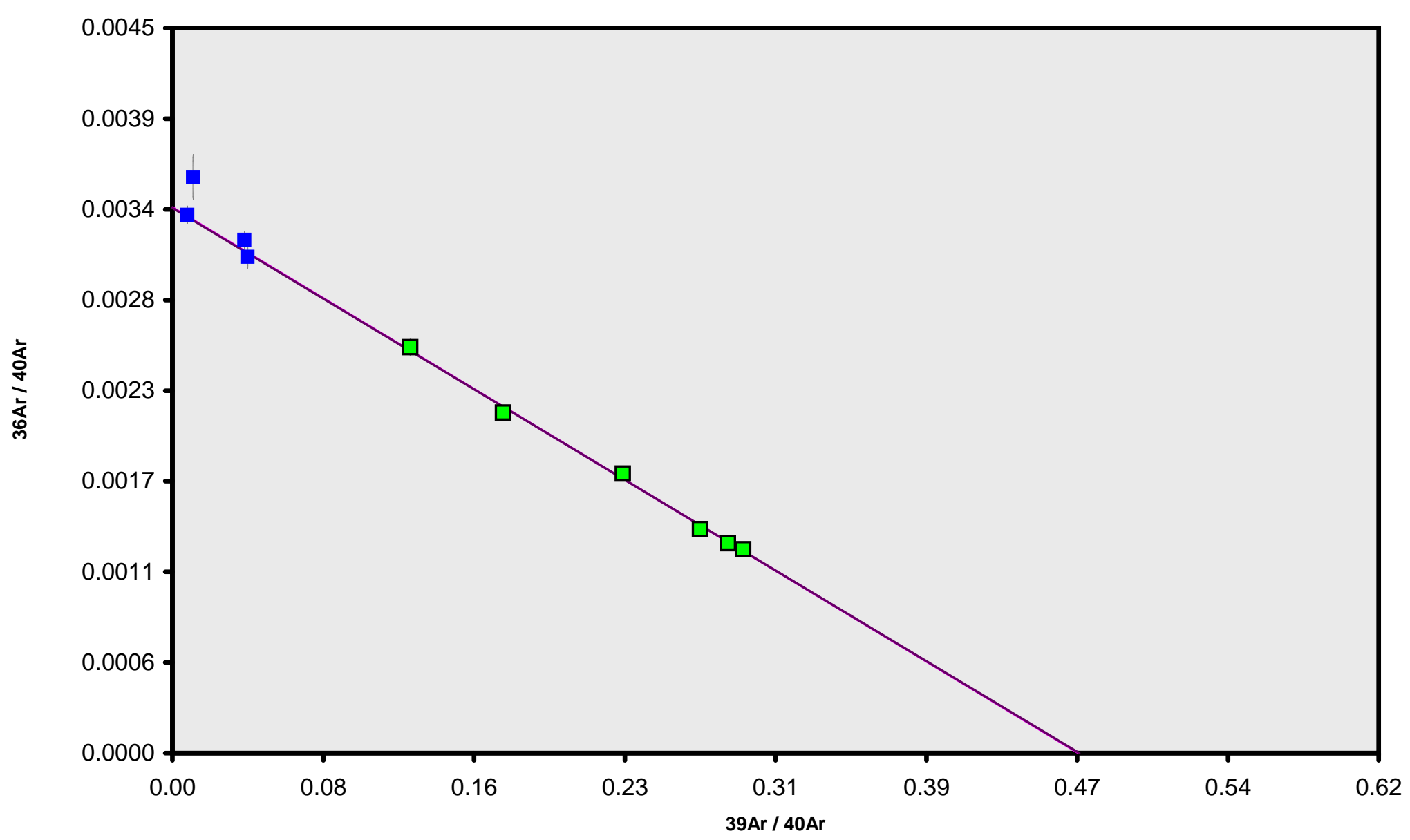

\section{Ar-Ages in $\mathrm{Ka}$}

Weighted Plateau

$1195.1 \pm 15.8$

Total Fusion

$1168.1 \pm 43.5$

Normal Isochron

$1196.2 \pm 34.5$

Inverse Isochron

$1196.1 \pm 34.6$

\section{MSWD}

2.04

Sample Info

bas grndmss

Westaway Volcanics

msp

cl155-

$0.0003103(\mathrm{~J})$ 